\title{
STUDY OF FOUR-LEPTON FINAL STATES IN ELECTRON-POSITRON INTERACTIONS AT $29 \mathrm{GeV}$
}

\author{
Assimina Petradza \\ Stanford Linear Accelerator Center \\ Stanford University \\ Stanford, California 94309
}

\begin{abstract}
August 1989
Prepared for the Department of Energy under contract number DE-AC03-76SF00515
\end{abstract}

Printed in the United States of America. Available from the National Technical Information Service, U.S. Department of Cornmerce, 5285 Port Royal Road, Springfield, Virginia 22161. Price: Printed Copy A07, Microfiche A01.

* partial fulfillment for $\mathrm{Ph}$. D. thesis 
As you set out for thake hope your road is a long one.

full of adventure, full of discovery.

Laistrygonians, Cyclops, angry Poseidon-don't be afratd of them:

gou'll never find things like that on your way

as long as you keep your thoughts raised high,

as long as a rave excitement stirs your opirit and your bady.

Larigirygonians, Cyclops, twild Poseidon-you won't encounter them

unless you bring them along inside your soul

uniess your soul sels them up in front of you.

Hope your road is a long one.

May there be mony summer mornings when,

with what pleassre, what joy,

you enter harbors you are seeing for the first time:

may you stop at Phoenician trading stations to byy fine things,

mother of pearl and coral, amber and etrony,

senswal perfume of every kind-as many senswal perfumes as you can;

and may you visit many Egyptian cities

to learn and go on learning from their scholars.

Keep Ithaka always in your mind. Arriving there is what you're destined for.

But don't harry the journey af all. Betler if it lasts for years,

so you 'se old by the time gou reach the island,

wealthy with all you've gained on the way,

not expecting Ithatea to make you rich.

Ithaka gave you the marvelous journey.

Without her you wauldn't have set out.

She has nothing left to give yas now.

And if you find her poor, Ithaka unn't have fooled you.

Wise as you will have becoma, so fell of experience,

you'tl have understood by then what these Ithakas mear. 


\begin{abstract}
This thesis presents a study of electron-positron scattering to four light leptons. The motivations behind it are twofold. Firstly, the study is a test of the theory of electron-positron interactions to 4 th order in the fine structure constant $\alpha$. A deviation from the theory could indicate the existence of a heavy new particle. Secondly, a measurement of these processes may prove useful in the understanding of other QED-type reactions. The method for simulating the four-lepton processes by the Monte Carlo event generator of Berends, Daverveldt and Kleiss is described. Theoretical predictions are compared to data from the Mark II and HRS experiments at the PEP storage ring. The observed events consist of four leptons at large angles. Data for all three $e^{+} e^{-} e^{+} e^{-}, e^{+} e^{-} \mu^{+} \mu^{-}$and $\mu^{+} \mu^{-} \mu^{+} \mu^{-}$processes are well described by the QED Monte Carlo calculation. The various kinematical distributions are in good agreement with QED to order $\alpha^{4}$.
\end{abstract}




\section{ACKNOWLEDGMENTS}

Six years in graduate school has been an interesting experience. Rudi Thun made it particularly educational, not only with the variety of projects that he assigned me, but also with his dedication as an advisor. It was he who suggested the subject of this thesis after discussions we bad with Martin Perl.

The members of the Mark II and HRS collaborations provided me with fine sets of data to work with, and valuable help when needed. I would like to express my special thanks to David Stoker from Mark II and David Blockus from HRS for the very many useful discussions riuring the course of this thesis. Chris Hawkins introduced me to the four-lepton Monte Carlo and Francois Le Diberder helped me understand its finer details. The SLAC theory group, and in particular Michael Peskin, often provided enlighteniag conments. The friendly belp of Charlotte Hee made problem-solving easier, especially where the Mark II software was concerned. Finally, the shape of this thesis owes a lot to Ray Cowan's expertise in TEX.

To my friends and family : thanks for sharing the journey ! 


\section{TABLE OF CONTENTS}

ABSTRACT ....................... . . . . . . . . . . .

ACKNOWLETGMENTS . . . . . . . . . . . . . . . . . . iii

LIST OF TABLES . . . . . . . . . . . . . . . . . . vii

LIST OF FIGURES . . . . . . . . . . . . . . . . . . . . . ix

LIST OF APPENDICES . . . . . . . . . . . . . . . . . . xii

\section{CHAPTER}

1 INTRODUCTION . . . . . . . . . . . . . . . I

2 THE MARK II DETECTOR AT PEP . . . . . . . . . 5

2.1 The PEP storage ring . . . . . . . . . . . . 7

2.2 Vertex and main drift chambers . . . . . . . . . 8

2.3 Time-of-flight counters . . . . . . . . . . . 10

2.4 Magnet . . . . . . . . . . . . . . . . . . 11

2.5 Liquid argon calorimeter . . . . . . . . . . . . . 11

2.6 Muor chambers . . . . . . . . . . . . . 14

2.7 End cap shower counters . . . . . . . . . . . . 15 
2.8 Trigger . . . . . . . . . . . . 16

3 THE HRS DETECTOR AT PEP . . . . . . . . . . . Is

3.1 Superconducting magnet . . . . . . . . . 18

3.2 Vertex chamber . . . . . . . . . . . . 20

3.3 Central and outer drift chambers . . . . . . . . 20

3.4 Electromagnetic calorimetry . . . . . . . . . . . 21

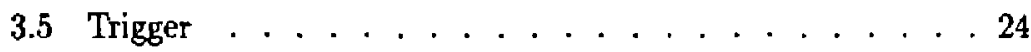

4 MONTE CARLO GENERATOR OF FOUR-LEPTON PROCESSES . . . . . . . . . . 2 ?

4.1 Theoretical description of four-lepton processes . . . . 28

4.2 Monte Carlo technique for event generation . . . . . . . 33

5 MONTE CARLC SIGNAL GENERATION AND ANALYSIS . . . . . . . . . . . . . . . 36

5.1 Event generation of four-lepton final states . . . . . 36
$5.1 .1 e^{+} e^{-} e^{+} e^{-}$ 38
$5.1 .2 e^{+} e^{-} \mu^{+} \mu^{-}$
$5.1 .3 \mu^{+} \mu^{-} \mu^{+} \mu^{-}$

5.2 Monte Carlo results . . . . . . . . . . . 44

5.2.1 Mark Il analysis of Monte Carlo signal events ............. . . 44

5.2.2 HRS analysis of Monte Carlo signal events

6 MONTE CARLO BACKGROUND

GENERATION AND ANALYSIS 
7 MARK II DATA ANALYSIS . . . . . . . . . . 57

7.1 Event selection and analysis ........... . 57

7.2 Monte Carlo event selection and comparison with data ............... . . . 66

7.3 Detection efficiencies . . . . . . . . . . . . 81

8 HRS DATA ANALYSIS . . . . . . . . . . . . . . 83

8.1 Selection criteria . . . . . . . . . . . . 83

8.2 Comparison with Monte Carlo . . . . . . . . . . . . . 89

8.3 Detection efficiencies . . . . . . . . . . . . 101

9 CONCLUSIONS . . . . . . . . . . . 103

APPENDICES ..................... 106

REFERENCES . . . . . . . . . . . . . . . ${ }_{i 27}$ 


\section{LIST OF TABLES}

4.1 Number of contributing diagrams in each of the final states . . . . . 32

5.1 Summary of MC event statistics and luminosities for Mark II . . . . 37

5.2 Summary of MC event statistics and luminosities for HRS . . . . . 47

i.l Effect of kinematical cuts on Mark II data in case (A) . . . . . . . 61

T.2 Effect of kinematical cuts on Mark Il data in case (B) . . . . . . . 62

7.3 Effect of analysis cuts on MC signal and background everits in case (A) 67

T.4 Effect of analysis cuts on $M C$ signal and background events in case (B) 68

7.5 Summary of Mark 11 results in case (A) . . . . . . . . . . . . . 71

7.6 Summary of Mark II results in case (B) . . . . . . . . . . . . . 72

7.7 Breakdown of $\mathrm{MC}$ identified events for case (A) . . . . . . . . . . 73

7.8 Breakdown of $\mathrm{MC}$ identified events for case (B) . . . . . . . . . . 74

7.9 Summary of Mark II cross sections and MC event statistics for case (A) 82

7.10 Summary of Mark II cross sections and MC event statistics for case (B) 82

8.1 Effect of the analysis cuts on HRS data . . . . . . . . . . . . . . 85

8.2 Effect of HRS analysis cuts on MC signal and background events . . 90

8.3 Summary of HRS results . . . . . . . . . . . . . . . . . . . . 92

8.4 Summary of HRS cross sections and MC event statistics . . . . . 102

9.1 Summary of $e^{+} e^{-} e^{+} e^{-}$results from various experiments . . . . . 104

9.2 Summary of $e^{+} e^{-} \mu^{+} \mu^{-}$results from various experiments . . . . . 105

A.l List of the generation parameters for $e^{+} e^{-} \epsilon^{+} e^{-} \mathrm{MC}$ events . . . . 110 
A.2 Summary of MC cross sections for $e^{+} e^{-} e^{+} e^{-}$events . . . . . . 112

A.3 Summary of MC efficiencies for $e^{+} e^{-} e^{+} e^{-}$events . . . . . . 113

C.1 Mark II four-lepton candidate events . . . . . . . . . . . . . 120

D.1 HRS four-lepton candidate events . . . . . . . . . . . . . 123 


\section{LIST OF FIGURES}

2.1 The Mark II detector at PEP . . . . . . . . . . . . . . . . . 6

2.2 The PEP storage ring . . . . . . . . . . . . . T

2.3 The Mark II/PEP5 drift chamber geometry . . . . . . . . . . . . 10

2.4 Mark II liquid argon calorimeter module . . . . . . . . . . . . . 13

2.5 Mark II ljquid argon calorimeter layer ganging scheme . . . . . . . 14

2.6 The Mark II muon system proportional chamber geometry . . . . . 15

3.1 The HRS detector at PEP . . . . . . . . . . . . . . . . . . . 19

3.2 The HRS central drift chamber . . . . . . . . . . . . . . . 22

3.3 Momentum resolution of the HRS . . . . . . . . . . . . 23

3.4 HRS barrel shower counter module . . . . . . . . . . . . . . . 25

4.1 The bremsstrahlung group of diagrams for $e^{t} e^{-} e^{+} e^{-} \ldots . . .30$

4.2 The annihilation group of diagrams for $e^{+} e^{-} e^{+} e^{-} \ldots \ldots$. . . . . . 31

4.3 The conversion group of diagrams for $e^{+} e^{-} e^{+} e^{-} \ldots \ldots$. . . . . . . 31

4.4 The multiperipheral group of diagrams for $e^{+} e^{-} e^{+} e^{-} \ldots \ldots$. . . . . 32

5.1 Momentum and angular distributions in $e^{+} e^{-} e^{+} e^{-}$MC events . . . 39

5.2 Angular distributions in $e^{+} e^{-} e^{+} e^{-} \mathrm{MC}$ events . . . . . . . . 39

5.3 Invariant mass distributions in $e^{+} e^{-} e^{+} e^{-}$MC events . . . . . . . 40

5.4 Momentum and angular disiributions in $e^{+} e^{-} \mu^{+} \mu^{-}$MC events . . 41

5.5 :.uggular distributions in $e^{+} e^{-} \mu^{+} \mu^{-}$MC events . . . . . . . . 42

5.6 Invariant mass distributions in $e^{+} e^{-} \mu^{+} \mu^{-}$MC events . . . . . . . 42 
5.7 MC lowest mass distributions . . . . . . . . . . . . . . 43

5.8 Mark II MC $e^{+} e^{-} e^{t} e^{-}$track and energy distributions . . . . . . 45

5.9 Mark II MC $e^{+} e^{-} \mu^{+} \mu^{-}$track and energy distributions . . . . . . . 46

5.10 Mark II MC $\mu^{-+} \mu^{-} \mu^{+} \mu^{-}$track and energy distributions . . . . . 46

5.11 HRS MC $e^{+} e^{-} e^{+} e^{-}$track and energy distributions . . . . . . 4S

5.12 HRS MC $e^{+} e^{-} \mu^{+} \mu^{-}$track and energy distributions . . . . . . . 49

5.13 HRS MC $\mu^{+} \mu^{-} \mu^{+} \mu^{-}$track and energy distributions . . . . . . 49

6.1 MC $e^{+} e^{-} \tau^{+} \tau^{-}$track multiplicity and energy distributions . . . . . 54

6.2 $\mathrm{MC} \epsilon^{+} e^{-h^{+} h^{-}}$track multiplicity and energy distributions . . . . 54

6.3 $\mathrm{MC} \tau^{+} \tau^{-}$track multiplicity and energy distributions . . . . . 55

6.4 MC hadronic track multiplicity and energy distributions . . . . . 55

6.5 $\mathrm{MC} e^{+} e^{-} \gamma \gamma$ track multiplicity and energy distributions . . . . . .56

7.1 Energy distribution of Mark II data before cut (3) . . . . . . . 60

7.2 Pair mass distribution of Mark II data before cut (4) . . . . . . . 60

7.3 Mass distribution of Mark II data before cut (5) . . . . . . . . . 62

$7.4 \mathrm{E} / \mathrm{P}$ distribution of Mark II four-lepton candidates . . . . . . 64

7.5 Distributions of $e^{ \pm}$in Mark II $e^{+} e^{-} e^{t} e^{-}$events . . . . . . . . 75

7.6 Distributions of $e^{ \pm}$in Mark $\mathrm{II} e^{+} e^{-} \mu^{+} \mu^{-}$events . . . . . . . . 76

7.7 Distributions of $\mu^{ \pm}$in Mark Il $e^{+} e^{-} \mu^{+} \mu^{-}$events . . . . . . . . . 76

7.8 Lowest mass distributions in Mark II $e^{+} e^{-} e^{+} e^{-}, \mathrm{c}^{+} e^{-} \mu^{+} \mu^{-} \ldots . .77$

7.9 Energy distributions in Mark II $e^{+} e^{-} e^{+} e^{-}, e^{+} e^{-} \mu^{+} \mu^{-} \quad \ldots . . .77$

7.10 Invariant mass distributions in Mark II $e^{+} e^{-} e^{+} e^{-}$events . . . . . . 78

7.11 Invariant mass distributions in Mark II $e^{+} e^{-} \mu^{+} \mu^{-}$events . . . . . 79

7.12 Mark Il $e^{+} e^{-} e^{+} e^{-}$event picture . . . . . . . . . . . . . . 79

7.13 Mark II $e^{+} e^{-} \mu^{+} \mu^{-}$event picture . . . . . . . . . . . . . 80

7.14 Mark II $\mu^{+} \mu^{-} \mu^{+} \mu^{-}$event picture . . . . . . . . . . . . 80

8.1 Energy distribution of HRS data before cut (2) . . . . . . . 86

8.2 Pair mass distribution of HRS data before cut (3) . . . . . . . . 87

$8.3 \mathrm{E} / \mathrm{P}$ distribution in HRS data . . . . . . . . . . . . . $8 \mathrm{SS}$ 
8.4 Energy distribution of tracks in HRS data . . . . . . . . . 88

8.5 Energy distribution of tracks with $\mathrm{E} / \mathrm{P} \leq 0.55$ in HRS data $\ldots . .89$

8.6 HRS $\alpha^{5}$ candidate event . . . . . . . . . . . . . . . . . . 91

8.T $\mathrm{E} / \mathrm{P}$ distribution in HRS data and $\mathrm{MC}$ events . . . . . . . . . . . 94

8.8 HRS data and MC energy distributions of tracks with $\mathrm{E} / \mathrm{P} \leq 0.55 \quad \cdot 94$

8.9 Distributions of $e^{ \pm}$in HRS $e^{+} e^{-} e^{t} e^{-}$events . . . . . . . . . .96

8.10 Distributions of $e^{ \pm}$in HRS $e^{+} e^{-} \mu^{+} \mu^{-}$events . . . . . . . . . . 96

8.11 Distributions of $\mu^{ \pm}$in HRS $e^{+} e^{-} \mu^{+} \mu^{-}$events . . . . . . . . 97

8.12 Lowest mass distributions in HRS $e^{+} e^{-} e^{+} e^{-}, e^{+} e^{-} \mu^{+} \mu^{-} \quad$. . . . . 97

8.13 Energy distributions in HRS $e^{+} e^{-} e^{+} e^{-}, e^{+} e^{-} \mu^{+} \mu^{-} \ldots \ldots 8$

8.14 Invariant mass distributions in HRS $e^{+} e^{-} e^{+} e^{-}$events . . . . . . . 98

8.15 Invariant mass distributions in HRS $e^{+} e^{-} \mu^{+} \mu^{-}$events . . . . . . . 99

8.16 HRS $e^{+} \varepsilon^{-} e^{+} e^{-}$event picture . . . . . . . . . . . . . . 99

8.1T HRS $e^{+} e^{-} \mu^{+} \mu^{-}$event picture . . . . . . . . . . . . . 100

8.18 HRS $\mu^{+} \mu^{-} \mu^{+} \mu^{-}$event picture . . . . . . . . . . . . . . 100

A.1 The center-of-mass energy distribution of MC events . . . . . . . 108

A.2 The distribution of $\mathrm{MC}$ generated cross section . . . . . . . . . 109

A.3 The distribution of $\mathrm{MC}$ observed cross section . . . . . . . . . 111

A.4 The distribution of MC effiriency . . . . . . . . . . . . . . . 114

B.1 E/P and energy distributions of Mark II end cap . . . . . . . 116 


\section{LIST OF APPENDICES}

Appendix

A RADIATIVE CORRECTIONS ........... 206

B STUDY OF THE MARK II END CAP SHOWER

COUNTER . . . . . . . . . . . . . . . 115

C MARK II EVENT LIS: . . . . . . . . . . . . . . . 118

D HRS EVENT LIST . . . . . . . . . . . . . . . . 122 


\section{CHAPTER 1}

\section{INTRODUCTION}

This thesis is a strdy of the production of four-lepton final states in $e^{+} e^{-}$interactions, using the Mark II and the HRS detectors at the Stanford Linear Accelerator Center. These detectors recorded data from events produced by the PEP storage ring, operating at a center-of-mass (c.m.) energy of $29 \mathrm{GeV}$.

During the last twenty years, $e^{+} e^{-}$experiments have led to many interesting and important discoveries. Although the main focus has been on processes like $e^{+} e^{-} \rightarrow$ $\mu^{+} \mu^{-}, e^{+} e^{-} \rightarrow e^{+} e^{-}, e^{+} e^{-} \rightarrow$ hadrons, where the interaction is medialed by a single, virtual photon, the interest in higher order processes, such as $e^{+} e^{-} \rightarrow e^{+} e^{-} e^{+} e^{+}$, $e^{+} e^{-} \rightarrow e^{+} e^{-} \mu^{+} \mu^{-}$or $e^{+} e^{-} \rightarrow e^{+} e^{-h}$ hadrons, has been increasing. In these processes, the dominant contribution to the total cross section comes from Feynman diagrams with two spacelike virtual photons which are emitted along the beam direction and are almost real. These diagrams are called multiperipheral, or t-channel diagrams. These processes are also known as two-photon processes, since the reactions are quasi-two body interactions of two almost-real photons. There is also a subclass of $O\left(\alpha^{4}\right)$ diagrams, namely those with two timelike virtual photons which, in addition 
to $e^{+} e^{-} \rightarrow e^{+} e^{-} e^{+} e^{-}, e^{+} e^{-} \rightarrow e^{+} e^{-} \mu^{+} \mu^{-}$and $e^{+} e^{-} \rightarrow e^{+} e^{-}$hadrons, can also yield final states consisting of $e^{+} e^{-} \rightarrow \mu^{+} \mu^{-} \tau^{+} \tau^{-}, e^{+} e^{-} \rightarrow \mu^{+} \mu^{-} \mu^{+} \mu^{-}$or $e^{+} e^{-} \rightarrow \mu^{+} \mu^{-} q \bar{q}$. When the timelike photons are massive, these non-multiperipheral processes are expected to have very small cross sections, since they probe higher order interactions at small distances. They offer an opportunity to make sensitive tests for unexpected physics.

It is the objective of this thesis to test QED to 4 th order in the coupling constant. $\alpha$ by studying those interactions of electrons and positrons which yield final states containing four observed light leptons :

$$
\begin{aligned}
& e^{+} e^{-} \rightarrow e^{+} e^{-} e^{+} e^{-} \\
& e^{+} e^{-} \rightarrow e^{+} e^{-} \mu^{+} \mu^{-} \\
& e^{+} e^{-} \rightarrow \mu^{+} \mu^{-} \mu^{+} \mu^{-}
\end{aligned}
$$

The cross section for these reactions is very small, of the order of a tenth of a picobarn $(p b)$, in the region of large pair masses and large scattering angles. In this region, the background to four-lepton final states is small, making them easily distinguishable despite the smaliness of the cross section.

By using data from two PEP detectors, Mark II and HRS, we are making two independent measurements of tha production of four-lepton final states in $\epsilon^{+} e^{-}$ interactions. These experiments accumulated large integrated luminosities. They also had different detector componerits, such as the muon detection system present only in the Mark II, thus allowing two independent tests of the theory.

The motivation of this analysis is the following : QED is the physical theory best established experimentally. It serves as the prototype of more evolved theories 
such as the electroweak theory and QCD. The degree of precision sttained in the measurements and predictions of $\mathrm{g}-2$ for the electron ${ }^{(1)}$ and the muon ${ }^{(3)}$ severely constrain the existence of new physics (3) ${ }^{(3 u c h}$ as composite leptons, excited leptons, and supersymmetric particles. The non-observation of significant deviacions from the theory in measurements of the differential cross sections of Bhabha scattering and muon-pair production estabiish QED to order $\alpha^{2}$ and $\alpha^{3}$ at small distances. The results reported here extend tests of $\mathrm{QED}$ to order $\alpha^{4}$ at large $Q^{2}$, where $Q^{2}$ is the four-momentum squared of the photon propagators appearing in tha Teynman diagran $s$ of the four-lepton final states. By requiring all leptons to be detected, one accomplishes two things. First, at large angies, virtual bremsstrahling processes are expected to dominate and the production of two virtual photons becornes measurable. Second, if massive new particles decaying into leptons are produced, an excess of events would appear above the QED prediction.

The proliferation of Feynman diagrams with jucreasing ordet make the calculations of QED contributions to order $\alpha^{4}$ quite difficult. For example, while the Bhabha reaction $e^{+} e^{-} \rightarrow e^{+} e^{-}$involves just 2 Feynman diagrams, final states with 4 electrons from $e^{+} e^{-}$interactions involve $36 !$ A Monte Carlo program, where all Feynman diagrams contributing to order $\alpha^{4}$ are taken into account, was written by Berends, Daverveldt and Kleiss ${ }^{(4-7)}$ to generate the four body final states. This is the one used in $\because: s$ analysis for comparison with the data.

In the past, other collaborations ${ }^{(8)}$ have done similar studies at c.m. energies ranging from 14 to $47 \mathrm{GeV}$ and found good agreement between data and QED 
predictions to $\alpha^{4}$. One group ${ }^{(9)}$ initially found some disagreement but recently reported agreement ${ }^{(10)}$ between their data and the Monte Carlo program of Beisnds, Daverveldt and Kleiss.

The further outline of this thesis is as follows, In the next two chapters we briefly describe the parts of the Mark II and HRS detectors which are relevant to this analysis. In chapter 4 we present the theoretical description of the Monte Carlo (MC) event generator used in the analysis. In chapter 5 we discuss the MC generation and simulation of signal processes. Expected backgrounds are discussed in the next chapter. Comparisons between theory and data from the Mark II and KRS experiments are then presented in chapters 7 and 8, respectively. Finally, in chapter 9 we summarize the results from Mark II and HRS and those reported by other experiments. 
CHAPTER 2

\section{THE MARK II DETECTOR AT PEP}

The Mark 11/PEP5 detector (12), shown in fig. 2.1, was a device built for studying the physics of $e^{*} e^{-}$interactions. After having been used initially at the SPEAR storage ring in 1978, it was placed at the PEP storage ring from 1980 through 1985. It was characterized by good charged particle tracking, electromagnetic calorimetry, and muon detection systems. An upgraded version of the detector is presently at the interaction region of the SLC for studying $Z^{\circ}$ physics.

After a brief description of the PEP storage ring, we present in this chapter a description of the components of the Mark II detector that are relevart to this andysis. Further details can be found in refs. 12 and 13. 


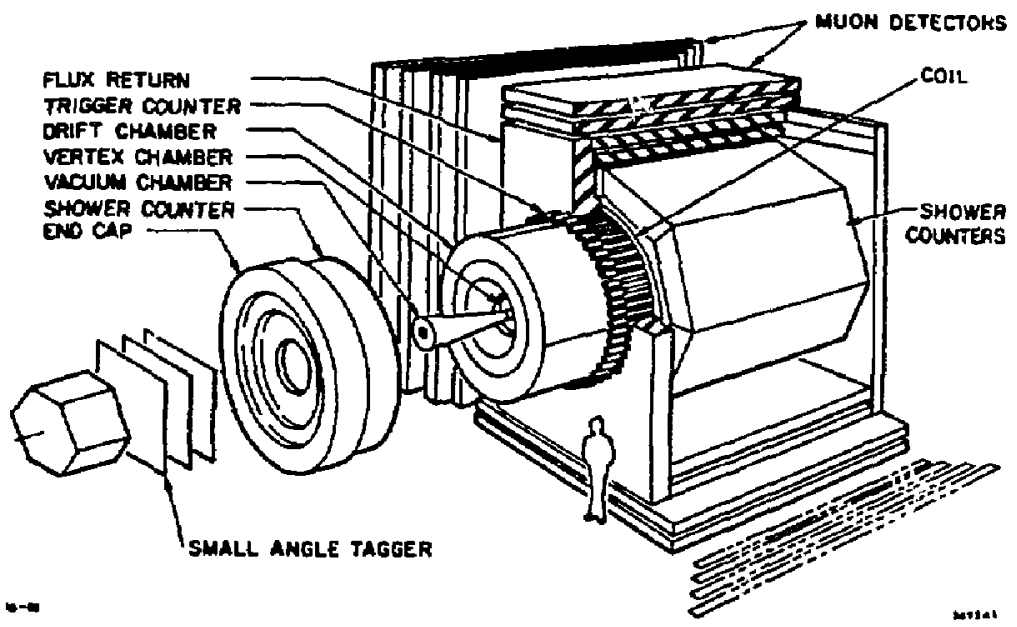

i)

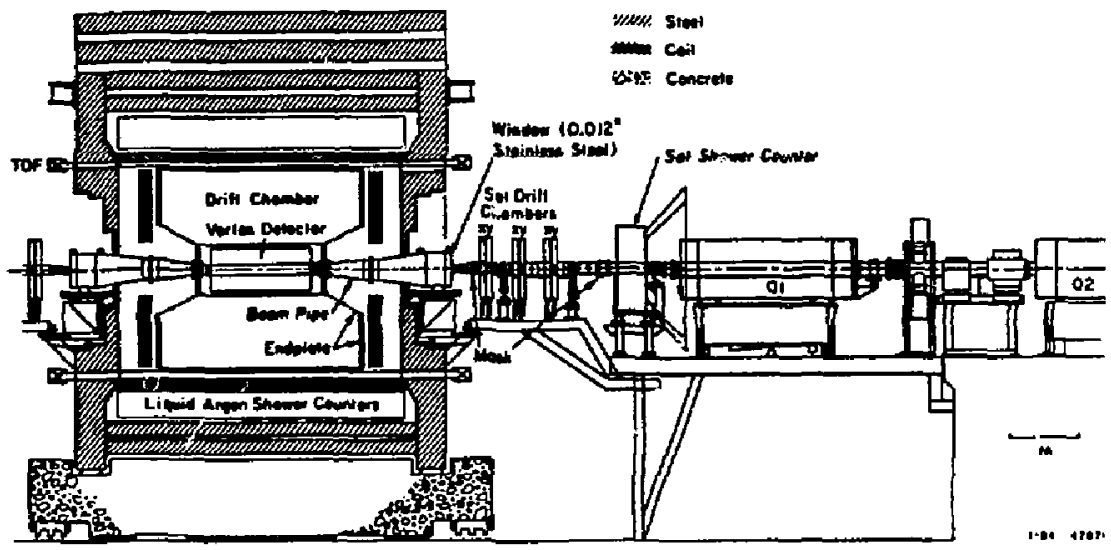

b)

Figure 2.1. The Mark II detector at PEP; a) isometric view; b) side view. 


\subsection{The PEP storage ring}

The PEP (Positron Electron Project) storage ring guides positrons and electrons around a ring with a diameter of $700 \mathrm{~m}$. The location of the PEP ring and the HRS and the Mark 11 detectors at the SLAC site is presented in fig. 2.2. Three positron and three electron bunches collided at six interaction regions, while circulating in opposite directions. The ring was run at $29 \mathrm{GeV}$, bur it had been originally designed for collision energies of up to $36 \mathrm{GeV}$. (14) This lower energy allowed operation at a higher luminosity. At each interaction region collisions occurred every $2.4 \mu \mathrm{s}$. The maximum luminosity reached was $3 \times 10^{31} \mathrm{~cm}^{2} \mathrm{sec}^{-1}$.

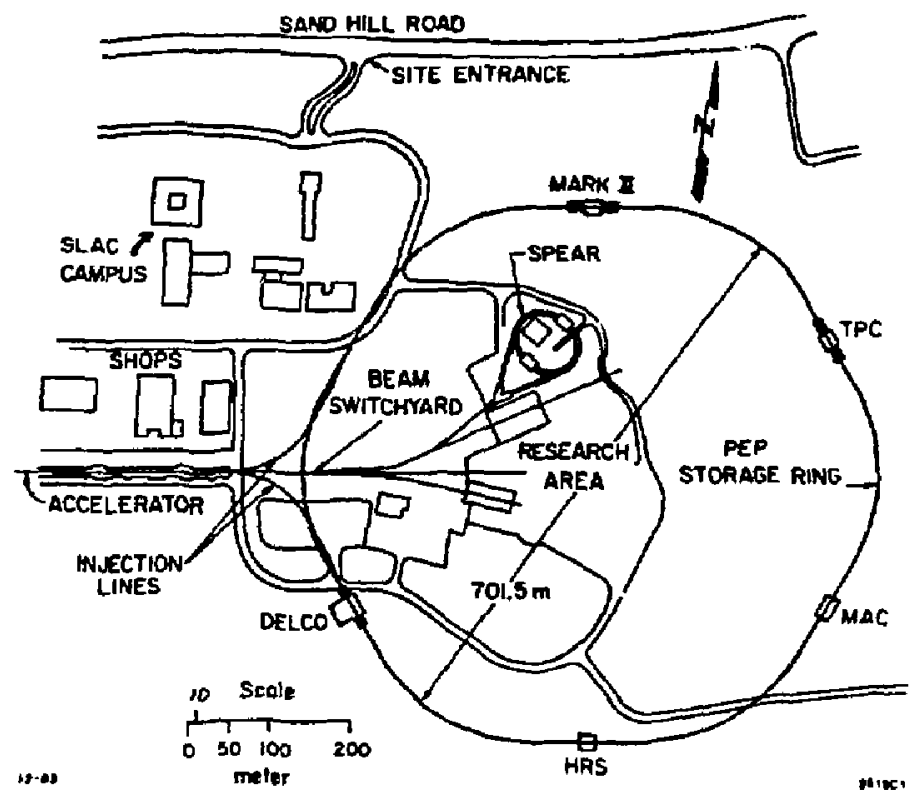

Figure 2.2. Overview of the PEP storage ring and the SLAC site. 


\subsection{Vertex and main drift chambers}

The inner part of the Mark II detector was the veriex chamber (15), a high precision cylindrical drift chamber, $126 \mathrm{~cm}$ long, with an outer radius of $35 \mathrm{crr}$. it was used to measure the distance of closest approach of particles to the evert origin, thereby improving momentum and lifetime measurements. It was built as close as possible to the $1.4 \mathrm{~mm}$ thick beryllium beam pipe, which formed the inner wall of the chamber.

The wires of this vertex chamber were organized in seven concentric layers. Four inner layers were at an average radius of $11.4 \mathrm{~cm}$ from the beam pipe, while the outer three were at a radius of $32.2 \mathrm{~cm}$. All wire; wese strurg axially, no ats $\$ \mathrm{mpt}$ being made to measure the $\mathrm{z}$ coordinate. This arrangement allowed the accurate projection of tracks back to the origin. There were 270 drift cells in the inner layers and 555 in the outer layers, with a radius of $0.53 \mathrm{~cm}$. Each cell was parallel to the axis and had a single sense wire. Apart from the sense wires that collected the ionization electrons, there were also field wires that carried high voltage and guard wires that ensured electrostatic stability. Field wires placed exactly between the sense wires minimized cell-to-cell cross talk. The chamber operated with a 50\%$50 \%$ mixture of argon and ethane at $\mathbf{1 5 . 5}$ psi.

The position resolution of the individual wires in the vertex chamber ranged from $85 \mu \mathrm{m}$ at the center of the drift cell up to $100 \mu \mathrm{m}$ at the edges. This excellent spatial resolution was due to :

1. the precise location of the wires

2. the minimization of Coulomb scattering due to the beryllium beam pipe, 
which was only $0.6 \%$ radiation length thick

3. the fact that the precision tracking in the vertex chamber was decoupled from that in the main drift chamber

4. an excellent timing resolution of $250 \mathrm{ps}$ of the electronics

5. the full efficiency of the chamber at the voltages used

6. the fact that the gas, kept at a stable pressure, maintained a constant drift velocity over the cell.

The $2.7 \mathrm{~m}$ long main drift chamber, ${ }^{(16)}$ shown in fig. 2.3 , measured the sign of the charge and the momentum of charged particles. Its sense wires were arranged in 16 concentric layers, located at various radij from the beam axis, ranging from $41.4 \mathrm{~cm}$ up to $144.8 \mathrm{~cm}$. The first six layers were located in a conical inner piece, made out of solid aluminum. The other ten were located on the outer flat endplates, which were made out of aluminum honeycomb. The drift cells in the conical piece were small, while the cells in the boneycomb portion were larger. All wires were made of silver plated beryllium-copper. Each cell had a single sense wire, surrounded by six field wires. The wires, strung in a $0^{\circ},+3^{\circ},-3^{\circ}$ pattern with respect to the axis of the detector, allowed the measurernent of the polar and azimuthal angles of the tracks. There were 3204 cells in the chamber, the large ones operating below the drift velocity saturation point, while the small ones operated just above it. The timing resolution of the electronics was $350 \mathrm{ps}$ and the average spatial resolution was $200 \mu \mathrm{m}$. The main drift chamber was operated with a $50 \%-50 \%$ mixture of argon and ethane, at atmospheric pressure. 


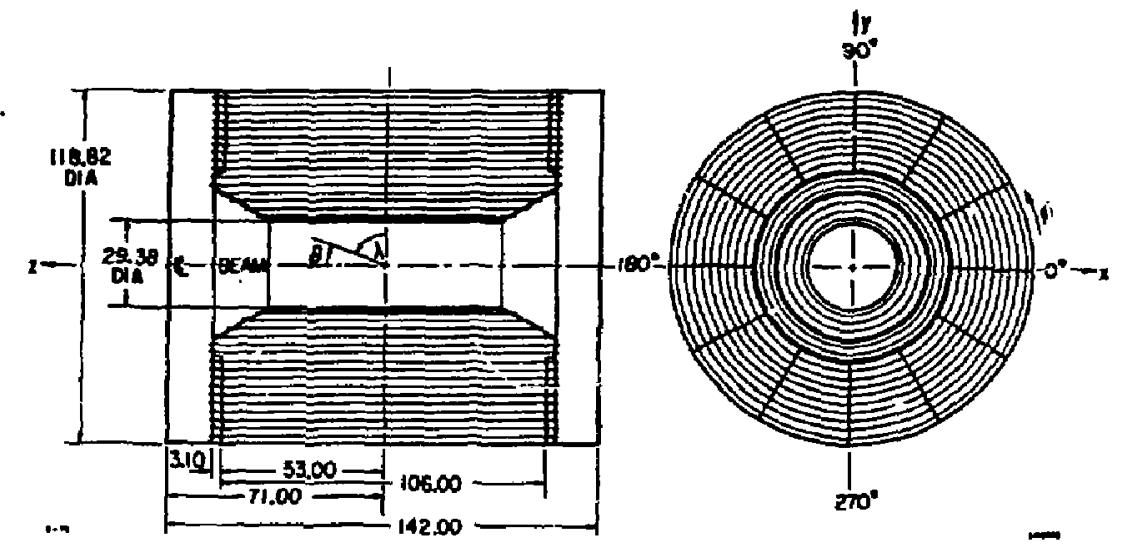

Figure 2.3. The Mark 11/PEP5 drift chamber geometry; the angles $\theta$ and $\phi$ are jefined.

The vertex and main drift chambers had a combined momentum resolution for

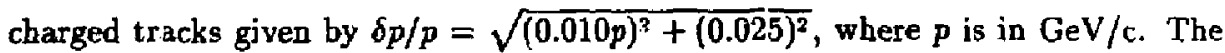
first term comes from the intrinsic resolution, the second from multiple scattering. The resolution was determined from Bhabha electron and cosmic ray studies.

\subsection{Time-of-flight counters}

The time-of-flight system, located around the main drift chamber and inside the solenoidal magnet, consisted of an array of 48 plastic scintillators, at a radius of $1.5 \mathrm{~m}$ from the beam pipe, each one $0.20 \mathrm{~m}$ wide and $3.4 \mathrm{~m}$ long. They covered $70 \%$ of the $4 \pi$ solid angle. Each counter was oriented along the beam axis. The light of the scintillator was brought to each end by a lucite light guide, and was collected there by a phototube. The output from these phototubes was fed into electronics that measured the charge integral signal and pulse arrival times.

The purpose of the time-of-flight system was the precise measurement of fight times of charged particles, to be used in the determination of the mass of slow 
particles. It was also used to eliminate cosmic ray backgrounds and to form an integral part of the charged-particle trigger. The timing resolution was about 350 $p s$, and the efficiency of having a time-of-tlight counter fired by a charged track was $99 \%$.

\subsection{Magnet}

The magnet, a conventional room temperature solenoid at a radius of $1.6 \mathrm{~m}$ from the beam axis, consisted of two water-cooled aluminum conductors separated by an insulating layer. It was external to the vertex and main drift chambers, as well as to the time-of-flight system. The conductors were 1.4 radiation lenghts thjck and produced a uniform axial solenojdal magne:ic field. The momentum of charged particles was deiermined from the curvature of their tracks in the magnetic field. Initially operated at $4.5 \mathrm{kG}$, it eventually developed a short between the inner and outer layers, due to corrosion caused by the cooling water. Only the outer conductor was powered from then on, yielding a $2.35 \mathrm{kG}$ field. Thus the momentum resolution was degraded, while the tracking of lower momentum particles became possible. The magnetic field was known to within $1 \%$.

\subsection{Liquid argon calorimeter}

An electromagnetic calorimeter, ${ }^{(17)}$ located outside the magnet coil at $1.8 \mathrm{~m}$ from the axis, (see fig. 2.1) measured the energies of showering particles and thereby distinguished electrons from other charged particles. It consisted of eight modules arrayed octagonally which covered $65 \%$ of the $4 \pi$ solid angle. The front portion of each module consisted of two aluminum planes, $1.6 \mathrm{~mm}$ thick, separated by $8 \mathrm{~mm}$ 
of liquid argon. These axial planes were known as the trigger gap and were $0.1 \%$ radjation lengths thick. They sampled the showers that started in the magnet coil. The rest of the module was a sandwich of $3 \mathrm{~mm}$ liquid argon gaps and $2 \mathrm{~mm}$ thick layers of lead, 37 layers in total, the whole assembly being coojed down to $88^{\circ} \mathrm{K}$. The add numbered layers were made of solid lead, while the even numbered layers were formed of lead strips and collected the ionization produced in the liquid argon. A potential difference of $3.5 \mathrm{kV}$ across the $3 \mathrm{~mm}$ liquid argon gaps provided a drift field for the released electrons. In order to determine the position of the shower, the layers of lead at high potential were segmented into strips in various directions (see fig, 2.4). Nine of these 18 readout strip layers, each of whick corresponded to 0.8 radiation lengths, had their $3.8 \mathrm{~cm}$ wide strips oriented axially, and gave information about the azimuthal angle of the shower. Six of them had their $3.8 \mathrm{~cm}$ wide strips perpendicular to the beam direction, and determined the polar angle. The remaining three planes had $5.4 \mathrm{~cm}$ strips oriented along the diagonal direction, and they helped resolve ambiguities concerning multiple showering tracks. There were 1152 lead strips all together in each module, and shower sampling occurred every $0.4 \%$ of a radiation length, the complete module being 14.4 radiation lengths thick.

The fine segmentation of the liquid argon made possible the precise determination of the shower position and led to a large number of electronic channels. The reduction of this number down to a more manageable number of the order of 3000 required the ganging of some strip planes, shown in fig. 2.5 , and the wiring iogether 


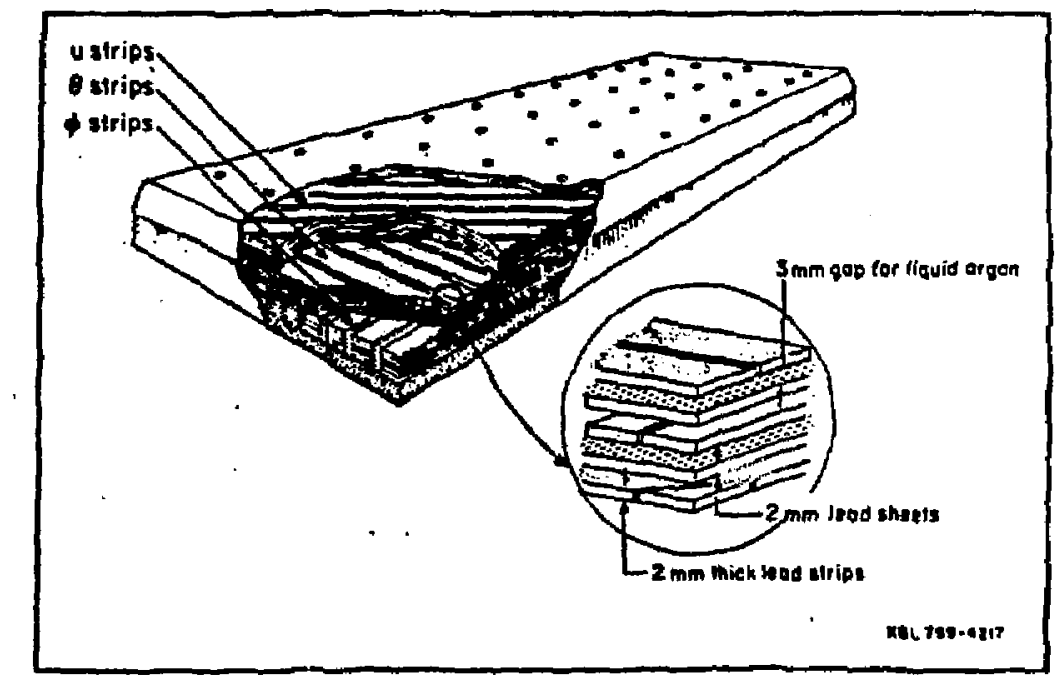

Figure 2.4. Cutaway view of a liquid argon calorimeter module. The ingert shows the liquid argon gap and the segmentation of the lead atrips.

of pairs of neighboring channels in the back of the calorimeter. Out of the 57 layers of lead, 18 were read out and ganged into six measurements, leaving a net number of 362 channels per module.

Due to the above described segmentation, both in depth and angle, the differentiation of electrons from hadrons became possible, since the electrons would shower quickly, depositing all their energy in the calorimeter, while most hadrons would pass through with little energy loss. The hadrons that did deposit energy in the calorimetex did so over a larger area than electrons. Furthermore, a very goad energy resolution of $14.5 \% / \sqrt{E}$ was achieved ( $\mathrm{E}$ in $\mathrm{GeV}$ ), while the entering position of a Bhabha electron covid be measured to within $8 \mathrm{~mm}$. The polar angle covered corresponded to $|\cos \theta| \leq 0.7$. The azimuthal angle covered was the $88 \%$ of $2 \pi$. 


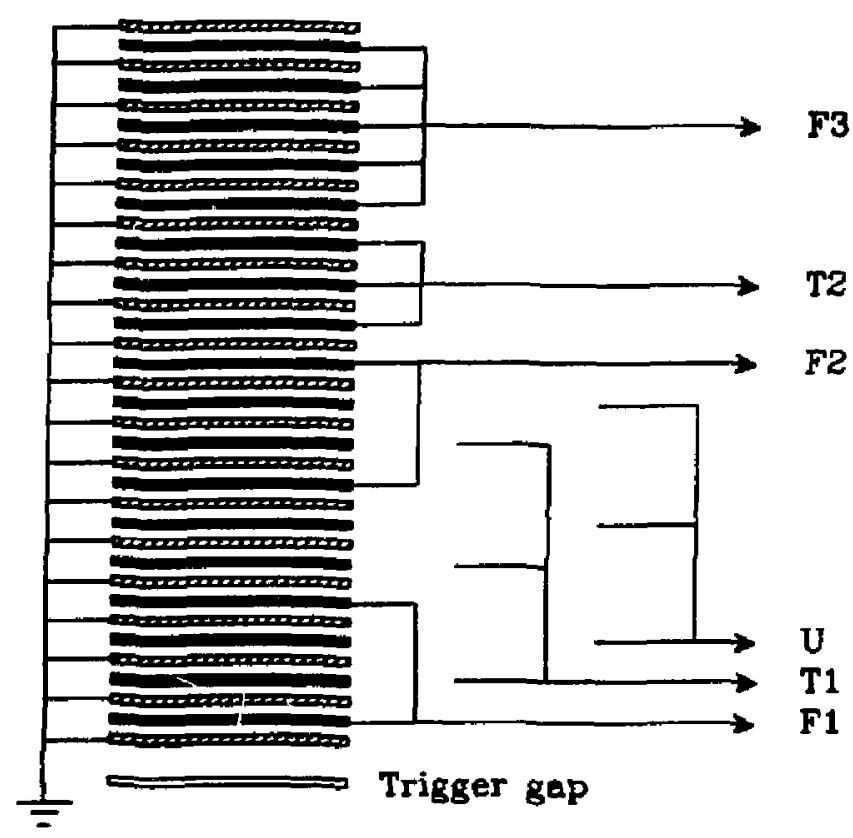

Figure 2.5. Liquid argon calorimeter ganging scheme. Particles enter from the trigger gap and the ionization is mensured along the aximuthal $(F)$, polar ( $T$ ), and diagonal (U) directions.

\subsection{Muon chambers}

The muon detection system, located outside the electromagnetic calorimeter, consisted of four walls placed at the top, bottom, right and left of the beam pipe, $235 \mathrm{~cm}$ away from the interaction point (see fig. 2.1). Each wall consisted of four layers of hadron absorber alternating with four layers of proportional tubes. The proportional tubes were made from extruded aluminum modules, each module having eight proportional wire chambers (see fig. 2.6). Each tube contained one $45 \mu \mathrm{m}$ wire, $2.5 \mathrm{~cm}$ away from the wire of the nearest proportional tube. There were 408 modules, 3264 channels, in total. The proportional wire chambers were triangular in shape, and operated at $2 \mathrm{kV}$ with a gas mixture of $95 \%$ argon and $5 \%$ carbon 
dioxide. The tubes in the first layer were perpendicular to the beam axis in order to measure the polar angle, while those in the outer three layers were oriented axially in order to measure the azimuthal angle. Particles had to traverse at least 7.4 interaction lengths of material in order to cross all the layers. This thickness ensured the reduction of the contamination from hadronic punchthrough, while accepting muons with a momentum as low as $1 \mathrm{GeV} / \mathrm{c}$. The complete system covered about $55 \%$ of the entire solid angle.

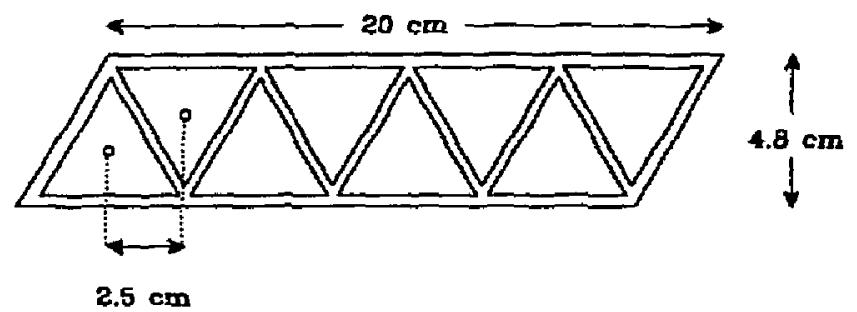

Figure 2.6. Mvon system rross sectional riew. A siogle module is shown.

\subsection{End cap shower counters}

Placed at each end of the drift chamber, the end cap shower counters covered the forward and backward regions of Mark II. They consisted of two sheets of lead, each one 2.3 radjation lenghts thick, alternating with two layers of proportional wire chambers. They measured electromagnetic showers between the polar angles of $15^{\circ}$ and $40^{\circ}$, over most of the azimuthal range. The system attained an energy resolution of $50 \% / \sqrt{E}$, tor photons and electrons, where $E$ is in $\mathrm{GeV}$. Its poor energy resolution, combined with the $2.5 \%$ probability of photon non-conversion, prevented 
its use in many Mark II/PEP analyses. In this analysis though, the system proved useful as a tool for discriminating electrons from muons as shown in appendix B.

\subsection{Trigger}

A two-level programmable trigger of considerable flexibility was used. It had very little dead time and could be reprogrammed for various event topologies. The primary trigger would decide if an event was interesting enough to be processed by the secondary trigger. It used simple selection criteria in the drift chambers, liquid argon and small angle shower counters. The primary trigger relevant to this analysis was generated by either charged tracking or calorimetry. The charged primary trigger was produced when a beam crossing signal coincided with a drift chamber majority signal (DCM). A DCM signal was present only if all of the following conditions were satisfied :

1. at least 3 vertex chamber layers hit

2. at least 6 drift chamber layers hit

3. at least 1 time-of-flight counter hit

The calorimetric primary trigger was generated by the presence of energy deposited in at least two Liquid Argon (LA) or End Cap (EC) modules. The energy threshold was $1 \mathrm{GeV}$ for $\mathrm{LA}$ and $2.5 \mathrm{GeV}$ for EC.

The secondary trigger was intended to find track patterns in the vertex and drift chambers and the time-of-flight system. A special pattern recognition processor, consisting of twenty-four curvature modules, was used to find tracks. A track was defined for the purposes of this trigger as a signal in two of the inner four vertex 
chamber layers, four inner and two outer main drift chamber layers, and a timeof-fight counter aligned in a momentum band. The generation of a secondary calorimetric trigger followed automatically if calorimetry had set the primary trigger. The secondary trigger requirement relevant to this analysis was that either the number of charged tracks or the number of calorimeter modules with total energy above a certain threshold be two or more. 


\section{CHAPTER 3}

\section{THE HRS DETECTOR AT PEP}

The High Resolution Spectrometer (HRS), ${ }^{(18)}$ shown in fig. 3.1, was a general purpose solenoidal detector. It was characterized by the strong uniform magnetic field (1.6 Tesla) of its superconducting magnet, a good spatial resolution of the drift chambers, a long tracking radius of $2 \mathrm{~m}$, and consequently an excellent momentum resolution for charged particles. The detector covered over $90 \%$ of the solid angle.

This chapter describes briefly the elements of the HRS detector which are relevant to this analysis, as they existed during the years 1982 through 1986, when data were taken at the PEP storage ring. Detailed descriptions of the HRS detector can be found in refs. 19 and 20.

\subsection{Superconducting magnet}

The unique characteristic of the HRS detector was its large superconducting magnet, with a cylindrical volume $4.8 \mathrm{~m}$ in diameter and $3.1 \mathrm{~m}$ in length. The solenoidal coil consisted of 15120 turns of niobium-titanium strands and was surrounded by an iron yoke of 1600 tons of low carbon steel. The magnet was operated at 1.6 Tesla during the collection of the data and was characterized by a uniform 


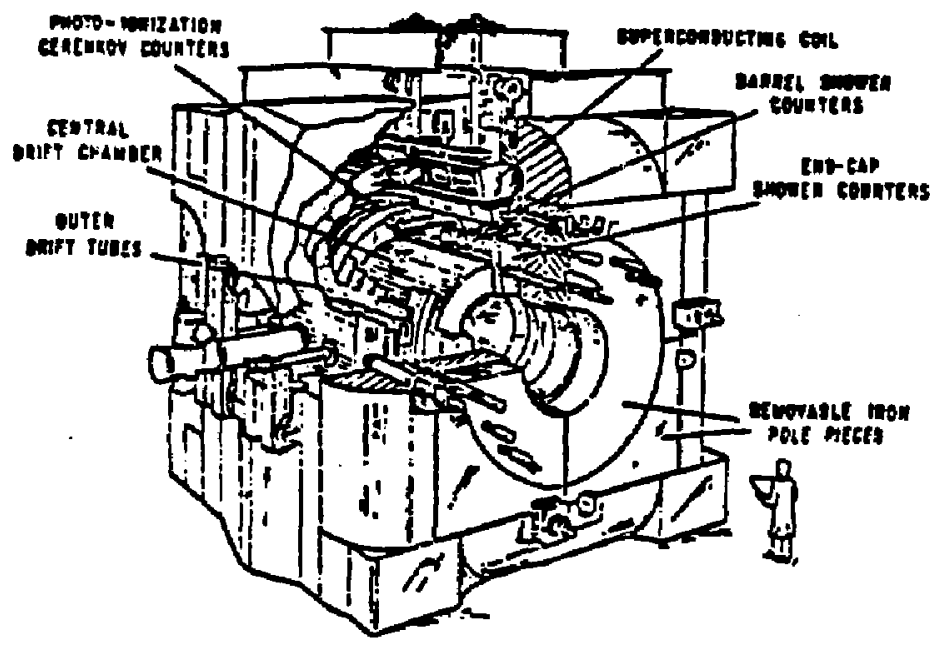

a)

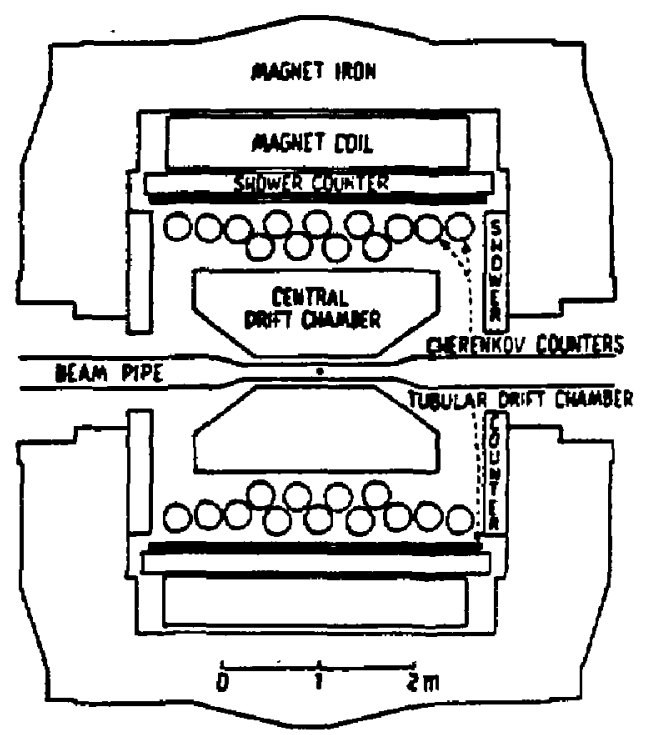

HRS DETECTOR - SIDE VIEW

b)

Figure 3.1. The BRS detector at PEP; a) isometric view; b) side view. 
field which varied less than $3 \%$ over the detector volume.

\subsection{Vertex chamber}

A small, bigh-precision drift chatnber was installed between the central drift chamber and the beam pipe after the first two years of running. It was made of two double layers of aluminized mylar tubes, ${ }^{(21)}$ and used a $75 \%$ argon - $25 \%$ ethane mixture at atmospheric pressure. As it was inside the main tracking systern, it was constructed with a total material thickness of just 0.004 radiation lengths to feduce multiple scattering. Its operation improved the track fitting, enhanced the momentum resolution, and made possible the accurate measurement of lifetimes of leptons and badrons. Since all the tracks had to pass through this chamber, it was also able to play the role of a cosmic ray veto, reducing the trigger rate by a factor of three. Its spatial resolution was $120 \mu \mathrm{m}$.

\subsection{Central and outer drift chambers}

Most of the tracking information was gathered by the central drift chamber, ${ }^{(22)}$ a large cylindrical chamber with 2448 drift cells distributed among fifteen concentric layers, as shown in fig. 3.2. The seven even-numbered layers had their wires placed parallel to the beam direction ( $z$, while the rest made a stereo angle of $\pm 60 \mathrm{mr}$ with respect to the beam axis. The cells in each layer numbered from 80 in the innermost layer at a radius of $21 \mathrm{~cm}$ to 256 in the outermast at a radius of $103 \mathrm{~cm}$. Each of these cells had a central sense wire carrying positive voltage, surrounded by six field shaping wires that carried negative voltage, yielding potential differences of about 2500 Volts. The drift cell efficiency exceeded $99 \%$ at 1.6 Tesla. A beryllium 
inner chamber wall and an aluminum honeycomb outer chamber wall reduced the multiple scattering. The total thickness of material traversed by particles was 0.02 radiation lengths. Charged particles were tracked over $90 \%$ of the entire solid angle. The spatial resolution was $200 \mu \mathrm{m}$. The gas used was a mixture of $89 \%$ argon, $10 \%$ carbon dioxide, and $1 \%$ methane (HRS gas). More details about the central drift chamber are described in Ref. 22.

The outer drift chamber, ${ }^{(23)}$ located $1.9 \mathrm{~m}$ away from the beams, was a cylindrical sheil consisting of 896 thin stainless steel tubes, arranged in two layers. The tubes were $350 \mathrm{~cm}$ long and had a $2.5 \mathrm{~cm}$ diameter. The layers were staggered by balf a tube width with respect to each other, to eliminate inefficiencies and resolve ambiguities. This chamber operated with HRS gas at 2150 volts and had a spatial resolution of $200 \mu \mathrm{m}$. Because the radius of the outer drift chamber was twice that of the inner drift chamber, the momentum resolution of large-angle tracks was improved by a factor of four as shown in fig. 3.3.

\subsection{Electromagnetic calorimetry}

Two sets of calorimeters were used to measure the energy and position of electromagnetic showers, as well as the time-of-flight of charged particles. The barre: shower counter system, with a time-of-flight resolution of \pm 360 ps and an energy: resolution of $16 \% / \sqrt{E}(E$ in $\mathrm{GeV})$, covered $60 \%$ of the entire solid angle. while the end cap shower counter system, with a time-of-Aight resolution of $\pm 1000 \mathrm{ps}$ and an: energy resolution of $20 \% / \sqrt{E}$ ( $E$ in $\mathrm{GeV}$ ), covered $27 \%$ of the total solid angie.

The barrel shower counter system, ${ }^{(24)}$, shown in fig. 3.4 , consisted of 40 muds:es: 


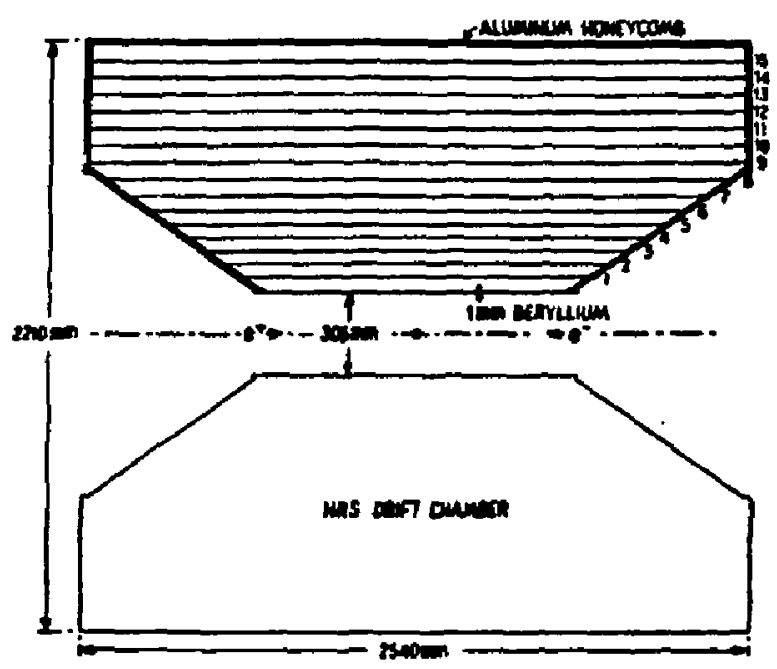

a)

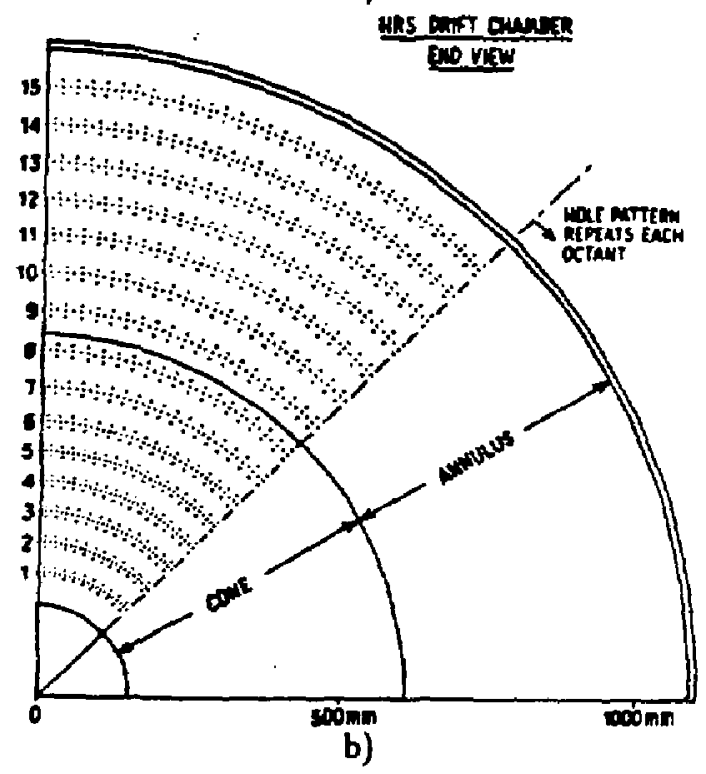

Figure 3.2. The BRS central drift chamber; a) side view; b) end view. 


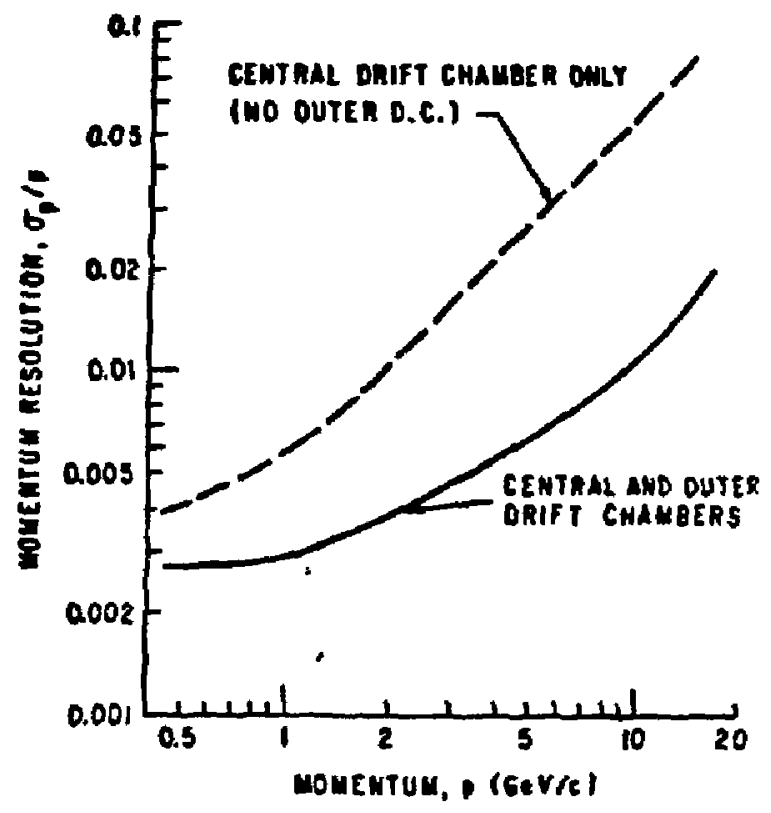

Figure 3.3. Momentum resolution of the HRS

of lead-scintillator sandwiches. These surrounded the outer drift chamber system, just inside the magnet coil, and formed a cylindrical shell coaxial with the beam line. Two sections, between which a layer of fourteen proportional wire chambers was placed, composed each module. Scintillation light from both the three radiation lengths thick front section and the eight radiation lengths thick back section was collected at the ends by two-inch diameter phototubes which provided energy information on the traversing particles. There were thus four phototubes per module, 160 in all. Processing the signals from these phototubes also provided time-of-tight information. The front section of each module consisted of two radiator plates of two and one radiation lengths, and two layers of scintillators. The outer section of 
the module had eight radiator plates, one radiation lengths thick, and eight layers of scintillators. All scintillators were $305 \mathrm{~cm}$ long, and were oriented axially. Segmentation along the azimuthal direction was provided by a total of 560 proportional wire cells. These cells were read out at both ends and provided $\mathrm{z}$ information by means of current division. Further details about the barrel shower counters are given in Ref. 24 .

The end cap shower counter system was composed of four C-shaped units mounted on the magnet return yoke at each end of the solenoid. Each unit contained 1.5 radiation lengths of lead, 76 proportional wire chambers oriented vertically, and 10 pie-shaped lead-scintillator sandwiches, arrayed in that order. These latter sandwiches consisted of eight layers of one radiation length thick lead sheets and $9.5 \mathrm{~mm}$ thick scintillators. They were viewed at their outer edge by one two-inch djameter phototube each. All proportional wire chambers used the HRS gas.

\subsection{Trigger}

The HRS trigger consisted of a two-level system similar to that of the Mark II. The primary trigger used the wire hit information from the central drift chambers to produce a charged trigger, and the phototube pulse height information from the shower counters to produce a neutral trigger. Within $1.5 \mu \mathrm{s}$ after a collision, the primary trigger decided whether an event should proceed to the secondary trigger or not.

If any of the following criteria was satisfied, the event was accepted by the primary trigger : 


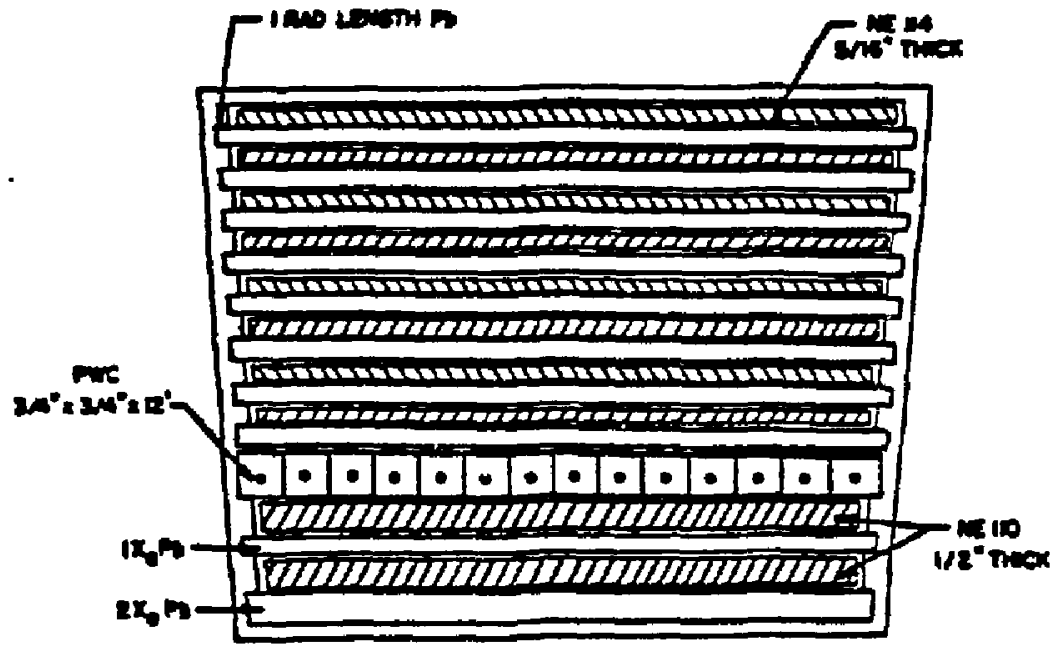

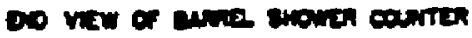

a)

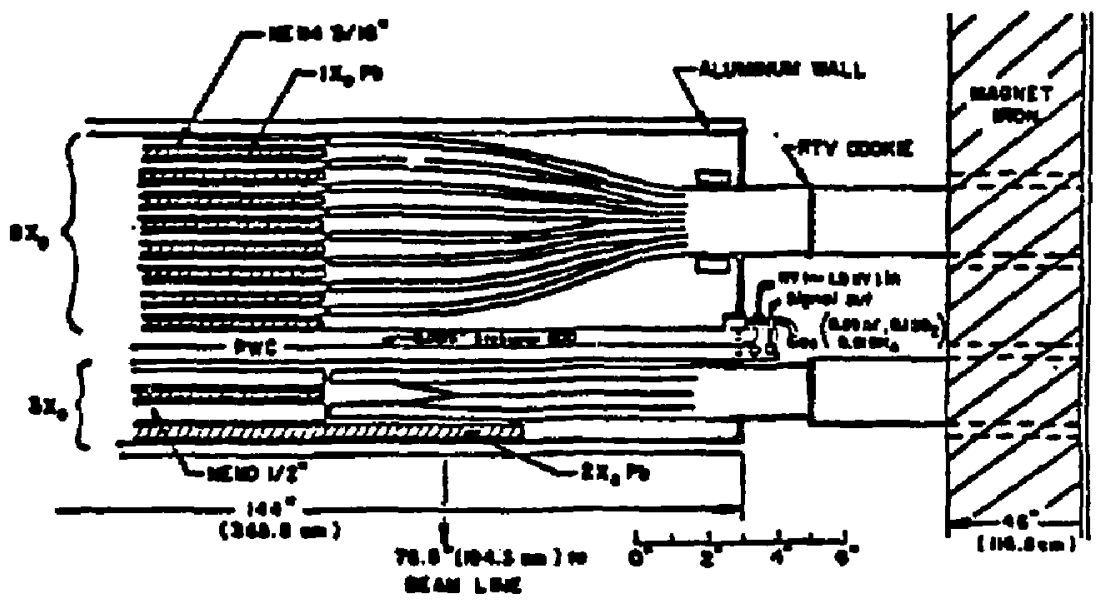

b)

Figure 3.4. HAS barrel shower counter module; a) end view; b) side view. 
- NT1: total energy deposited in the end cap and barrel shower counters $>4.8 \mathrm{GeV}$

- NT2: total energy deposited in the end cap and barrel shower counters $>2.4 \mathrm{GeV}$

- CT1: $\geq 12$ layers of the central drift chamber bit

- CT2: $\geq 7$ of the inner 8 layers of the central drift chamber plus a hit in the corresponding quadrant in the end cap shower counter

- SP5: back to back hits in opposite end cap modules

- SP6: a phototube signal in the shower counters within an 80 ns gate SP5 and SP6 were not used after the installation of the vertex chamber.

If the event was rejected by the primary trigger, the detector would get ready for the next collision. Otherwise the secondary trigger was activated. A 24-module track finding system, the curvature processor, was then used to search for drift chamber hits forming track segments, and to determine their approximate momenta.

The secoudary trigger, after completing the track finding within $39 \mu \mathrm{s}$, accepted the event provided any of the following requirements was satisfied :

- NT1

- NT2 plus at least one track found

- 2 tracks found in the vertex chamber

- 3 to 6 tracks found without the requirement of the vertex chamber.

Accepted events were written onto tapes within several ms, producing a typical dead time of about $6 \%$ for the detector. 


\section{CHAPTER 4}

\section{MONTE CARLO GENERATOR OF FOUR-LEPTON PROCESSES}

The classical linear equations of electrodynamics do not allow electromagnetic waves to be scattered, because the superposition principle requires that the 'rays traverse one another without hindrance'.(25) Quantum Electrodynamics (QED), however, predicts that electromagnetic waves interact nonlinearly through the creation and absorption of virtual fermion pairs. (26-28)

It was Euler ${ }^{(27)}$ who first calculated elastic photon-photon scattering, while Landau and Lifshitz ${ }^{(29)}$ computed the cross section for the production of $e^{+} e^{-}$pairs from collisions of charged particles fifty-five years ago. It was only recently that cross sections for four-lepton final states were calculated by Vermaseren ${ }^{(30)}$ and by Berends, Daverveldt and Kleiss. ${ }^{(4-7)}$ Vermaseren wrote a Monte Carlo integration program v'inich only included diagrams of the type of the $e^{+} e^{-} \mu^{+} \mu^{-}$final states shown in figs. 4.1-4.4. The four-electron final state actually requires a total of thirty-six diagrams !

The full leading-order calculation, taking all the diagrams into account, has been doae by Berends, Daverveldt and Kleiss. The comparison of data with theory in this thesis are based on the Monte Carlo programs by Berends et al., which are 
especially designed to generate four-lepton final states where all four leptons are emitted at large angles. In these programs, Berends et al. have taken into account both photon and $Z^{0}$ exchanges to order $\alpha^{4}$. At c.m. energies of $29 \mathrm{GeV}$, the $Z^{0}$ contribution to the cross sections is very small because of the presence of the large $Z^{0}$ mass in the progagators.

\subsection{Theoretical description of four-lepton processes}

Quantum electrodynamics is a field theory where the interacting part of the Lagrangian has a small coupling constant. This particularly fortunate feature of the theory is the one that enables us to calculate the cross sections for various processes, and compare them to experiment, in contrast with QCD. The reason is that we can use perturbation theory. In the context of quantum field theory, perturbation theory is best expressed in terms of Feynman diagrams, the sum of which gives the transition amplitudes for the various processes. The sums of these Feynman djagrams can be expected to converge only if the coupling constant is small enough. Even in the case of QED, where the series is asymptotic, we can use such perturbation techniques due to the smallness of the coupling constant.

In this and subsequent sections we concentrate on four-lepton final states where only photon exchanges are considered. The object of this thesis is the comparison of data with the calculation of the cross section for processes of the type $e^{+} e^{-} \rightarrow$ $l^{+} l^{-} L^{+} L^{-}$, where $l, L$ are electrons or muons. This calculation involves a large number of diagrams. Furthermore, the computation of each amplitude involves complicated combinations of Dirac matrices. The complexity of these calculations 
necessitates the use of numerical algorithms. These algorithms have been presented in the series of papers by Berends, Daverveldt and Kleiss that were mentioned above. The differential cross section for these processes can be evaluated using the corresponding amplitudes since

$$
d \sigma=\frac{\alpha^{4}}{128 \pi^{4} E_{b}^{2}}|M|^{2} \delta^{4}\left(p_{+}+p_{-}-q_{+}-q_{-}-k_{+}-k_{-}\right) \frac{d^{3} \vec{q}_{+} d^{3} \vec{q}_{-} d^{3} \vec{k}_{+} d^{3} \vec{k}_{-}}{q_{+}{ }^{0} q_{-}{ }^{0} k_{+}{ }^{0} k_{-}{ }^{\circ}} .
$$

The amplitude $M$ of the process is equal to the sum of the amplitudes of all the relevant diagrams, where each amplitude can be evaluated using the standard Feynman rules. Here $p_{+}$and $p_{-}$are the incoming momenta, and $q_{+}, q_{-}, k_{+}, k_{-}$are the outgoing momenta. These transition amplitudes have to be calculated numerically before squaring and summing over the spins. Such numerical calculations necessitate the systematic classification of the 36 diagrams describing the process $e^{+} e^{-} \rightarrow e^{+} e^{-} e^{+} e^{-}$, or of the 12 diagrams describing $e^{+} e^{-} \rightarrow e^{+} e^{-} \mu^{+} \mu^{-}$, or of the 12 diagrams describing $e^{+} e^{-} \rightarrow \mu^{+} \mu^{-} \mu^{+} \mu^{-}$. This classification is made necessary because of the extreme variations of the differential cross section. Indeed, in the $e^{+} e^{-} e^{+} e^{-}$case there are 657 different poles of the differential cross section in a phase-spacc with seven dimensions! As each peak must be describable by a set of integration variables, and as there is no single set of integration variables that can do the job for all the diagrams, Berends et al. ${ }^{(4-3)}$ divided the 36 diagrams of the $e^{+} e^{-} e^{+} e^{-}$case into 4 groups.

Group (I) is the 'bremsstrahlung' group, shown in fig. 4.1 for $e^{+} e^{-} e^{+} e^{-}$final states, where one of the photons has a four-momentum squared $Q^{2}>0$, and the other $Q^{2}<0$. They are most important in the reactions examined in this thesis. 

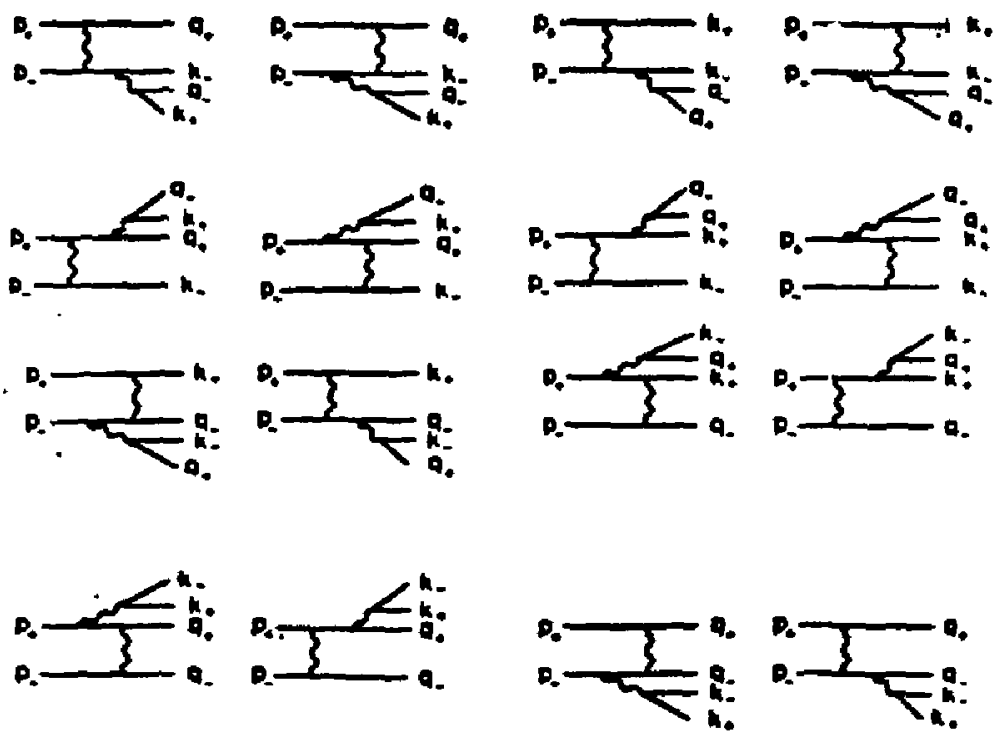

Figure 4.1. The brematrahlung group of diagrarns for $e^{+} e^{-} e^{+} e^{-}$. The diagrama in the

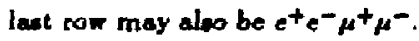

Groups (II) and (III) are the 'annikilation' and 'conversion' groups, respectively, and are shown in fig. 4.2 and fig. 4.3 for $e^{+} e^{-} e^{+} e^{-}$final states. Both photons satisfy $Q^{2}>0$. They can be neglected as soon as one of the electrons is emitted at small angles. However, for the large-angle reactions studied in this thesis they represent a significant effect.

Group (IV) is the 'multiperipheral' group, shown in fig. 4.4 for $e^{+} e^{-} e^{+} e^{-}$final states. Both photons satisfy $Q^{2}<0$. This group of diagrams becomes dominant as soon as one of the electrons is emitted at small angles. Of the four classes, the multiperipheral group contributes the least to the four-lepton final states in this study. 

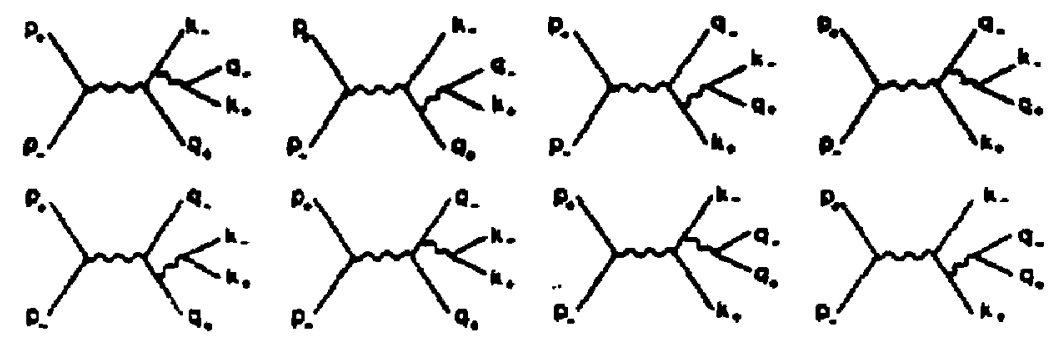

Figure 4.2. The annibilation group of diagrams for $e^{+} e^{-} e^{+} e^{-}$. The diagrams in the last row may also be $\mathrm{e}^{+} e^{-} \mu^{+} \mu^{-}$.
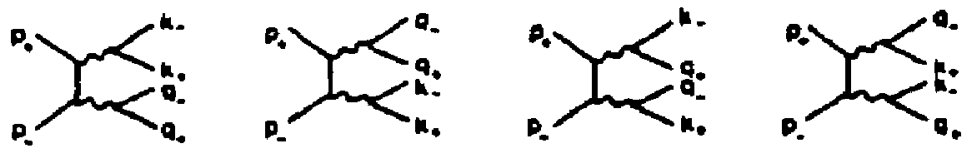

Figure 4.3. The conversion group of diagrams for $e^{+} e^{-} e^{+} e^{-}$. The first two diagrams may also be $e^{+} e^{-} \mu^{+} \mu^{-}$.

The number of diagrams that contribute in each of the final states, where only photon exchanges are accounted for, is shown in table 4.1 . When, in addition, the $\mathrm{Z}^{0}$ exchanges are taken into account, the number of contributing diagrams increases by a factor of four. 

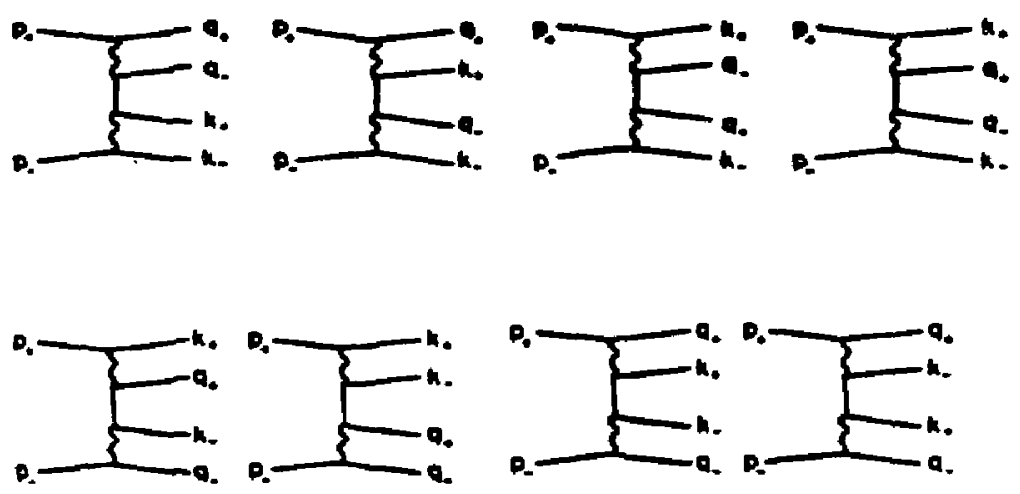

Figure 4.4. The muitiperipheral group of disgrams for $e^{+} e^{-} e^{+} e^{-}$. The lant two diagrams may also be $e^{+} e^{-} \mu^{+} \mu^{-}$.

Table 4.1. Number of contributing diagrams in each of the find atates.

\begin{tabular}{|c|c|c|c|c|c|}
\hline & Group (I) & Group (II) & Group (III) & Group (IV) & total \\
\hline$e^{+} e^{-} e^{+} e^{-}$ & 16 & 8 & 4 & 8 & 36 \\
\hline$e^{+} e^{-} \mu^{+} \mu^{-}$ & 4 & 4 & 2 & 2 & 12 \\
\hline$\mu^{+} \mu^{-} \mu^{+} \mu^{-}$ & 0 & 8 & 4 & 0 & 12 \\
\hline
\end{tabular}




\subsection{Monte Carlo technique for event generation}

Theoretical predictions and experimental results are usually compared at the cross section level. In the past, the relative simplicity of the detectors and the low order of the examined processes made possible theoretical prediclions consisting of just a number, such as the value of the cross section. Modern detectors, however, are composed of complicated subsystems with diverse responses. Furthermore, when going to higher orders, the cross section formulae become complex functions of many variables and the calculations are not straightforward. The replacement of a single predicted number by an event generator is thus vital for two reasons. Firstly, it is necessary to apply different selection criteria on the various subsystems of a particular detector, in order to make reliable measurements. Secoridly, it is essential to be able to simulate any experimental setup in order to allow for cross checks of the theory.

The presence of a very large number of poles in the differential cross section of four-lepton final states calls for a special Monte Carlo techgique. Berends et al. used 'importance sampling'. This method is unique for removing singularities in the expression for a differential cross section, a task achieved through the use of a sampling function. This fanction must be analytically integrable and must moreover exhibit the same peaking structure as the exact expression $d \sigma$. At this point, the seemingly artificial djvision of the total number of diagrams into 4 groups is justified, since every group has its own characteristic peaking behaviour. For each group (bremsstrahlung, annihilation, conversion, or multiperipheral), a separate subgenerator is designed. The exact differential cross section $d \sigma_{i}$ of a particular group $i$ is 
calculated.

Within a group there are sets of diagrams which form gauge invariant combinations and are denoted as subgroups. For each subgroup $j$, a suitable approximation $d \tilde{\sigma}_{j}$ describing the same peaking behaviour as the exact differential cross section, as well as a set of appropriate integration variables, are specified. Interferences between subgroups $j$ within a group $i$ are also calculated. The approximate cross section $\hat{a}_{i}$ can be thus calculated for each of the four groups.

An event is generated according to a subgenerator $i$ which is selected at random, and an approximate $d \tilde{\sigma}_{i}$ is calculated. A weight which equals the ratio of the exact over the approximate differential cross section, $W_{i}=\frac{d o_{j}}{d \sigma_{i}}$, corresponding to the particular subgenerator $i$ used, is initially assigned to the event.

At this stage, the decision about keeping or rejecting an event is based on the following algorithm : if $R \times W_{\max }<W_{i}$, where $R$ is a random number between zero and one and $W_{\max }=\max _{i=1,4}\left\{W_{\max }\right\}$, the event is accepted as an unweighted event. The cross section calculated up to this point is simply given by $\sum_{i=1,4} d \sigma_{i}$. The choice of $W_{\max }$ is dictated by efficiency requirements. Berends et al. have set all of them equal, but the user is free to give them arbitrary values. In our work we have kept their assignments. If $R \times W_{\max }>W_{i}$, the event is rejected and the process for the generation of a new event is repeated.

Next, interferences between the four groups are accounted for. The ratio

$$
I=\frac{\sum_{i=1,4} d \sigma_{i}}{d \sigma}=\frac{\sum_{i=1,4}\left|M_{i}\right|^{2}}{\left|\sum_{i=1,4} M_{i}\right|^{2}}
$$

is formed, where $M_{i}$ is the complete matrix element corresponding to the subgener- 
ator $i$. We use the symbol $I_{\max }$ for a predetermined maximum value that the ratio $I$ is allowed to take. If $r \times l_{\max }<J$, where $r$ is a random number between zero and one, then the calculation of the total exact cross section may proceed.

The event $n$ is assigned a new weight $W_{n}=\max \left(I, I_{\max }\right)$. Finally, the average of the weights $\langle W\rangle_{i}$ for all those events $n$ that are generated according to the subgenerator $i$ is calculated. The multiplication of $\widetilde{\sigma}$, the total approximate cross section given by $\sum_{i=1,4} \tilde{\sigma}_{i}$, by the average of the weights for all the generated events gives the total exact cross section $\sigma$, which bas the simple form

$$
\sigma=\sum_{i=1, ., 4} \frac{N_{i}}{N}(W)_{i} \tilde{\sigma}
$$

Here $i$ runs over the four subgenerators, while $N$ is the total number of generated events and $N_{i}$ is the number of events generated according to the subgenerator $i$.

In principle, the Monte Carlo event generator described above can simulate any experimental set-up. In the version we used, the scattering angles of the beam particles were chosen as integration variables, thus ensuring that not too many events were thrown away after the imposition of appropriate cuts.

In the next chapter we present a list of the parameters used for the event generation and the kinematical cuts applied after the event generation, as well as event statistics for the three examined processes $e^{+} e^{-} e^{+} e^{-}, e^{+} e^{-} \mu^{+} \mu^{-}$and $\mu^{+} \mu^{-} \mu^{+} \mu^{-}$. Comparisons of the theoretical predictions to the experimental data from two PEP experiments, Mark II and HRS, are presented in chapters 7 and 8, respectively. 


\section{CHAPTER 5}

\section{MONTE CARLO SIGNAL GENERATION AND ANALYSIS}

A precise comparison of a theoretical cross section with the data requires a program to simulate the detector response. It is therefore necessary that the generated particles, the momenta of which are distributed according to the theoretical cross section, be passed through a detector simulation program, so that the limitations of the detector can be taken into account. In this chapter we outline a study of Monte Carlo generated signal events for the purpose of choosing appropriate selection criteria. Results of event statistics and comparisons to Mark II and HRS data are presented in chapters 7 and 8 , respectively.

\subsection{Event generation of four-lepton flnal states}

Particle momenta for all three processes $e^{+} e^{-} e^{+} e^{-}, e^{+} e^{-} \mu^{+} \mu^{-}$and $\mu^{+} \mu^{-} \mu^{+} \mu^{-}$ were generated by using the Berends, Daverveldt and Kleiss ${ }^{(4)}$ event generator designed for four-lepton processed, when all four leptons are emitted at large angles, as discussed earlier in chapter 4. All Feynman diagrams contributing to lowest order were taken into account. All possible virtual photon and all possible $Z^{0}$ exchanges were included to order $\alpha^{4}$. The kinematic range of the generated particles extended 
a few standard deviations beyond the final acceptance criteria. The kinematical requirements on the generated events were applied in two steps. The first step demanded the scattering angle of the final-state particles to be within the angular interval $20^{\circ} \leq \theta \leq 160^{\circ}$ with respect to the electron beam direction. The second step imposed the following two conditions :

- All particles were required to have a momentum $P$ of at least $0.1 \mathrm{GeV} / \mathrm{c}$.

- All opposite-charge particle pair combinations were required to have an invariant mass $M_{\text {pair }}$ of at least $0.5 \mathrm{GeV} / \mathrm{c}^{2}$.

The event statistics and integrated luminosities of the MC generated events for each of the processes $e^{+} e^{-} e^{+} e^{-}, e^{+} e^{-} \mu^{+} \mu^{-}$and $\mu^{+} \mu^{-} \mu^{+} \mu^{-}$are summarized in table 5.1. The distributions presented in this section have been normalized to $205 \mathrm{pb}^{-1}$, the Mark II detector's integrated luminosity.

Table 5.1. Surnmary of MC event statistics and integrated luminosities for Mark II.

\begin{tabular}{|c|c|c|c|}
\hline & \begin{tabular}{c|c} 
Events \\
$\left(p b^{-1}\right)$
\end{tabular} & $\begin{array}{c}\text { Events satisfying } \\
\text { satisfying } \\
20^{\circ} \leq \theta \leq 160^{\circ}\end{array}$ & $\begin{array}{c}\mathbf{P} \geq 0.1 \mathrm{GeV} / \mathrm{c} \\
\mathrm{M}_{\text {poir }} \geq 0.5 \mathrm{GeV} / \mathrm{c}^{2}\end{array}$ \\
\hline$e^{+} e^{-} e^{+} e^{-}$ & 6005 & 5284 & 2686 \\
\hline$e^{+} e^{-} \mu^{+} \mu^{-}$ & 3682 & 4160 & 1853 \\
\hline$\mu^{+} \mu^{-} \mu^{+} \mu^{-}$ & 28343 & 1171 & 688 \\
\hline
\end{tabular}




\section{$5.1 .1 e^{+} e^{-} e^{+} e^{-}$}

It is interesting to examine the kinematical distributions of Monte Carlo generated $e^{+} e^{-} e^{+} e^{-}$final states, before any detector simulation is done. In this and subsequent subsections we shall use the term 'electrons' to denote both $e^{+}$and $e^{-}$, and the term 'muons' to refer to both $\mu^{+}$and $\mu^{-}$. The momenturn distribution of the final-state electrons is displayed in fig. 5.1 (a), while fig. 5.1 (b) shows the angular distribution. The distributions peak at low and high momenta, and at angles corresponding to the beam direction.

We now present the relative angular position of the four tracks in correlation to the magnitude of their momenta. Fig. 5.2 (a) shows the distribution of the angle $\theta(1,2)$ between those two tracks that have zero net charge and the highest momenta in the event. This is to be compared with the distribution of the angle $\theta(3,4)$ between the other two tracks in the event, shown in fig. $5.2(\mathrm{~b})$. We see that the two most energetic electrons in the event are almost back to back, as expected frorn momentum conservation. The two least energetic electrons are generally close to each other in angle. This is an indication that bremsstrahlung is an important process. The conversion and annihilation diagrams can also give similar results, depending on the value of the four-momentum squared $Q^{2}$ of the photons. 

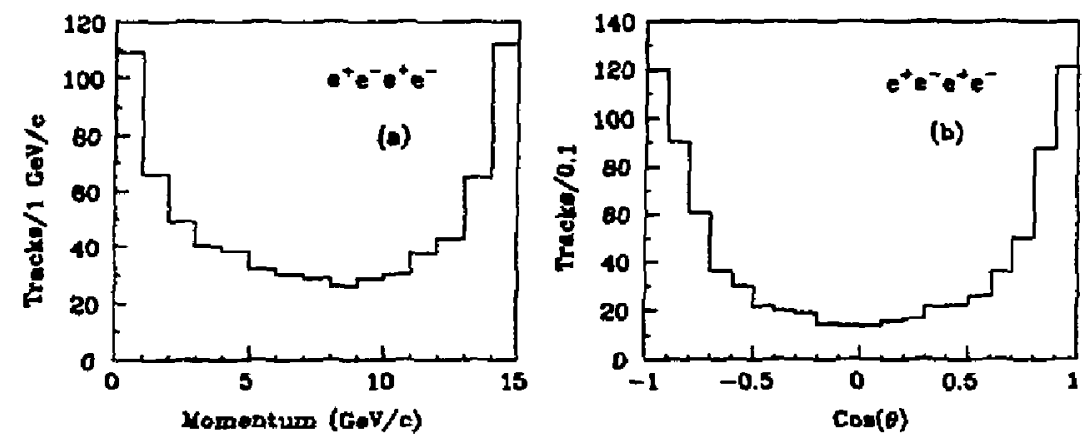

Figure 5.1. Distribution of electrons $\left(e^{ \pm}\right)$in $e^{+} e^{-} e^{+} e^{-}$MC events in momentum (a) and $\cos \theta$ (b).
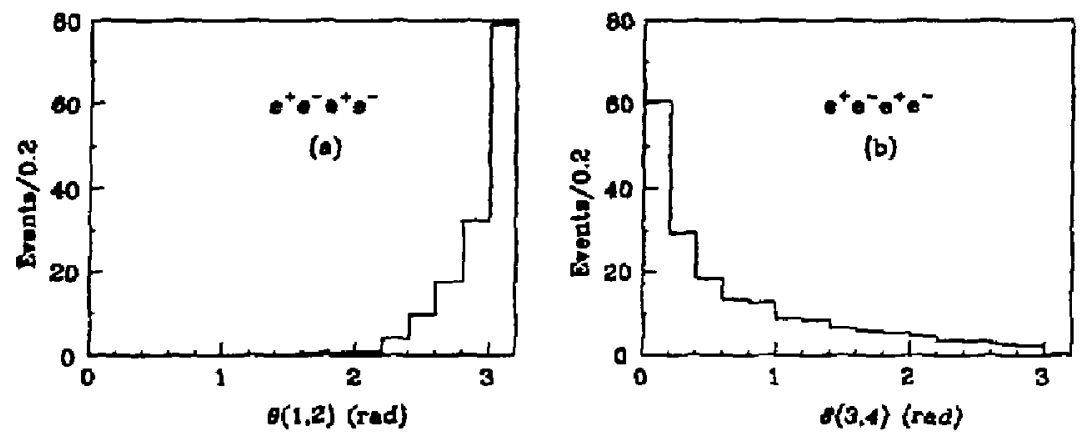

Figure 5.2. Angulas distribution of electrons $\left(e^{ \pm}\right)$in $e^{+} e^{-} e^{+} e^{-M C}$ events : (a) $\theta(1,2)$ and (b) $\theta(3,4)$ (see text). 
The distributions of invariant masses of the four $e^{+} e^{-}$pairs in each event are shown in fig. 5.3 (a) (d) in increasing mass order. Fig. 5.3 (a) shows the invariant. mass distribution of the $e^{+} e^{-}$pair with the smallest mass among the four pairs, whereas fig. 5.3 (d) shows the distribution of $e^{+} e^{-}$pairs with the largest invariant mass. Momentum conservation requires that a pair with large invariant mass appear (fig. 5.3 (d)) whenever a pair with small invariant mass appears (fig. 5.3 (a)).

When all four electrons are emitted in the angular interval $20^{\circ} \leq \theta \leq 160^{\circ}$ with respect to the electron beam direction, the total $e^{+} e^{-} e^{+} e^{-}$exact cross section is $(0.88 \pm 0.01) \mathrm{pb}$ before momentum and pair mass cuts. More than about $85 \%$ of the total cross section comes from bremsstrahlung type events.
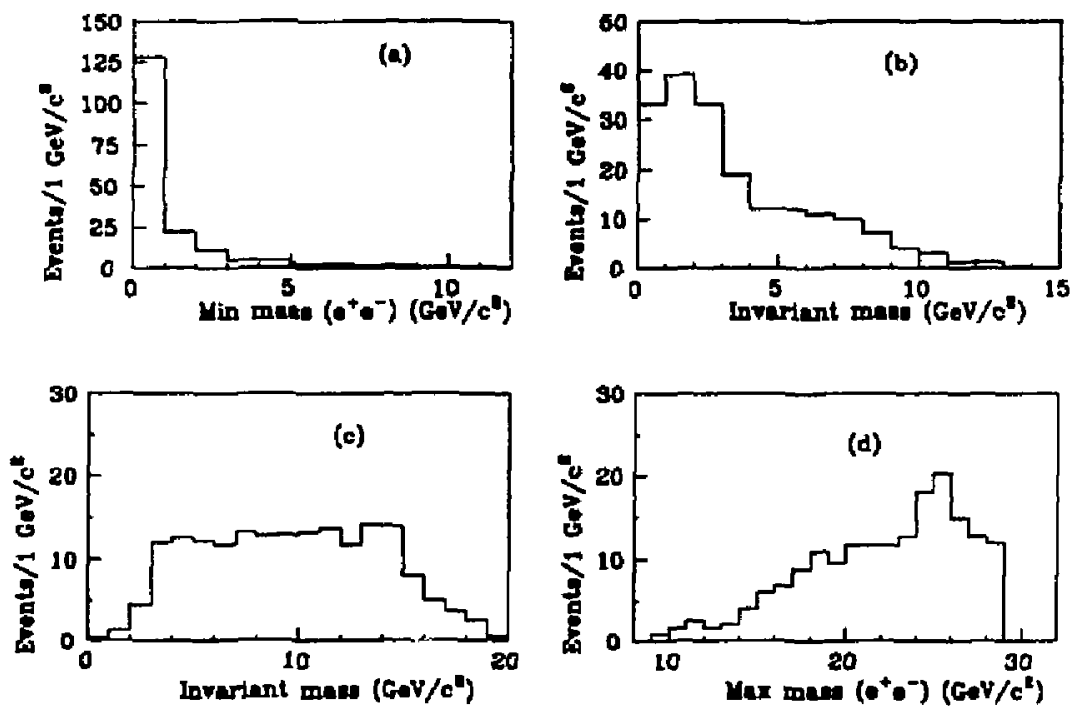

Figure 5.3. Invariant mas distribution in $e^{+} e^{-} e^{+} e^{-}$events for all $e^{+} e^{-}$pairs in increasing mass order (a)-(d). 


\section{$5.1 .2 e^{+} e^{-} \mu^{+} \mu^{-}$}

The momentum and angular distributions before detector simulation of all four final-state particles in $e^{+} e^{-} \mu^{+} \mu^{-}$are shown in fig. 5.4. These distributions are similar to those seen in $e^{+} e^{-} e^{+} e^{-}$processes. We also present the distributions of the opening angles of the $e^{+} e^{-}$pair and of the $\mu^{+} \mu^{-}$pair in fig. 5.5 (a)-(b).
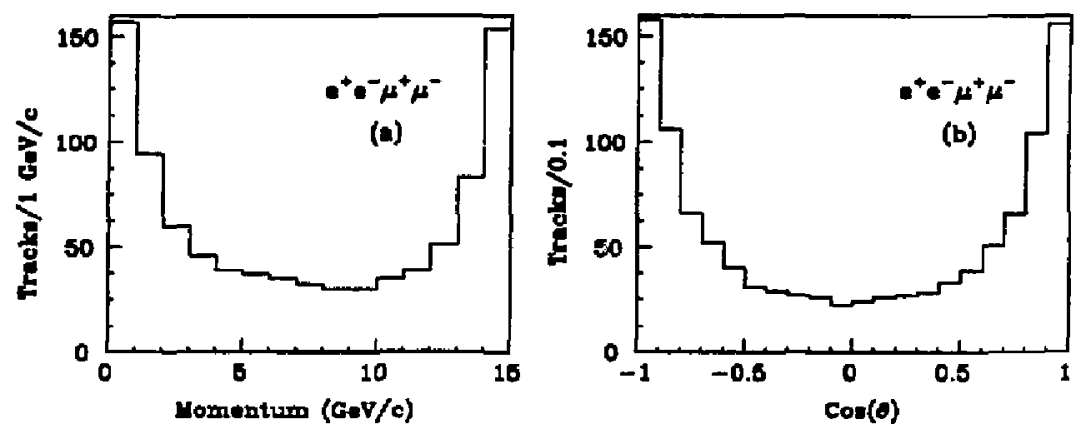

Figure 5.4. Distribution of tracks $\left(e^{ \pm}, \mu^{ \pm}\right)$in $e^{+} e^{-} \mu^{+} \mu^{-}$MC events in momentum (a) and $\cos \theta$ (b).

The invariant mass distributions of the $e^{+} e^{-}$and the $\mu^{+} \mu^{-}$pairs are shown in fig. 5.6 (a) and (b), respectively. The masses of the electrons and muons have been set to zero in the calculations of invariant masses. This approximation has a negligible impact on the analysis.

Finally, we compare the minimum invariant mass distribution of two leptons in both cases : $e^{+} e^{-} e^{+} e^{-}$events and $e^{+} e^{-} \mu^{+} \mu^{-}$events. The lowest invariant mass of all four possible combinations $e^{+} e^{-}$in $e^{+} e^{-} e^{+} e^{-}$events is shown in fig. 5.7 (a), whereas fig. 5.7 (b) presents the lower of the invariant masses of the $e^{+} e^{-}$or $\mu^{+} \mu^{-}$ 

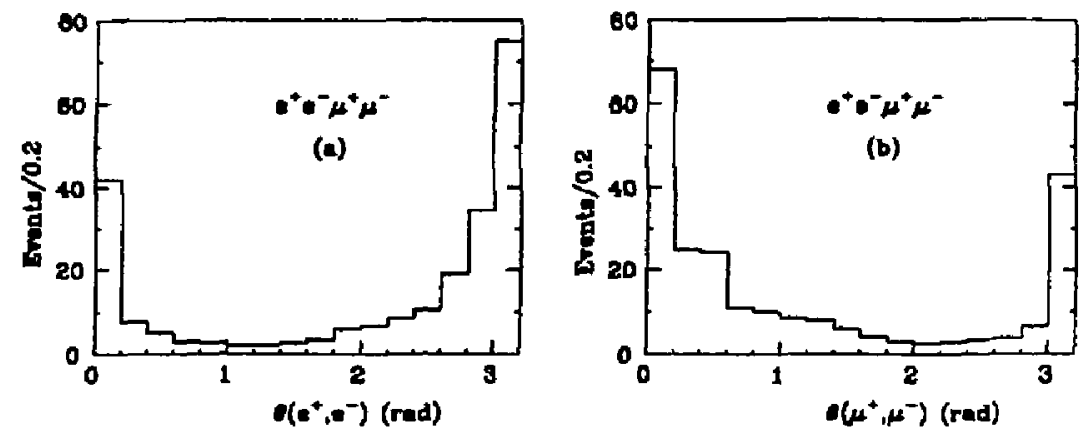

Figure 5.5. Angular distribution of tracks $\left(e^{ \pm}, \mu^{ \pm}\right)$in $e^{+} e^{-} \mu^{+} \mu^{-}$MC events : (a) $\theta\left(e^{+}, e^{-}\right)$and (b) $\theta\left(\mu^{+}, \mu^{-}\right)(\sec t e x t)$.
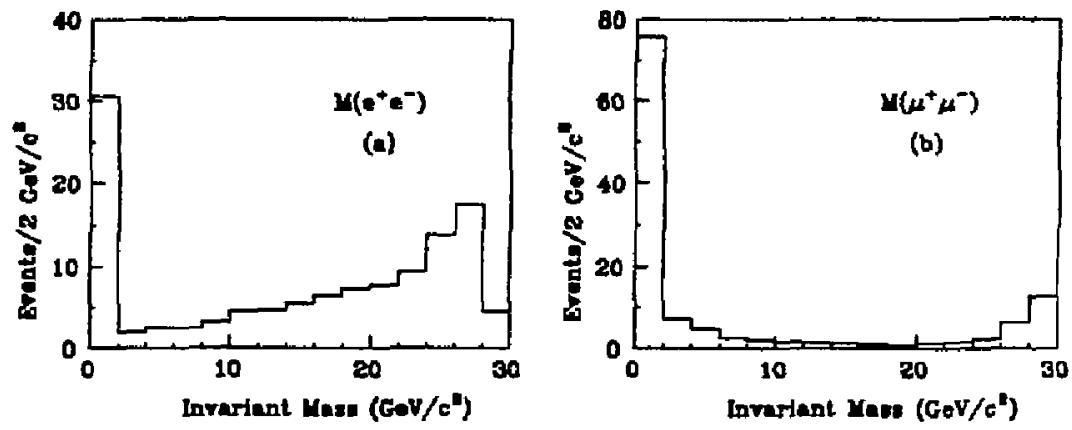

Figure 5.6. Invariant mass distributions of the $e^{+} e^{-}$pair (a) and of the $\mu^{+} \mu^{-}$pair (b) in $e^{+} e^{-} \mu^{+} \mu^{-}$events.

pairs in $e^{+} e^{-} \mu^{+} \mu^{-}$events. The distributions are very similar.

When all four final-state particles are emitted in the angular interval $20^{\circ} \leq \theta \leq 160^{\circ}$ with respect to the electron beam direction, the total exact cross section of generation of the $e^{+} e^{-} \mu^{+} \mu^{-}$final state is (1.14 \pm 0.01$)$ pb before momentum and pair mass cuts. In this final state, the bremsstrahlung contribution is 

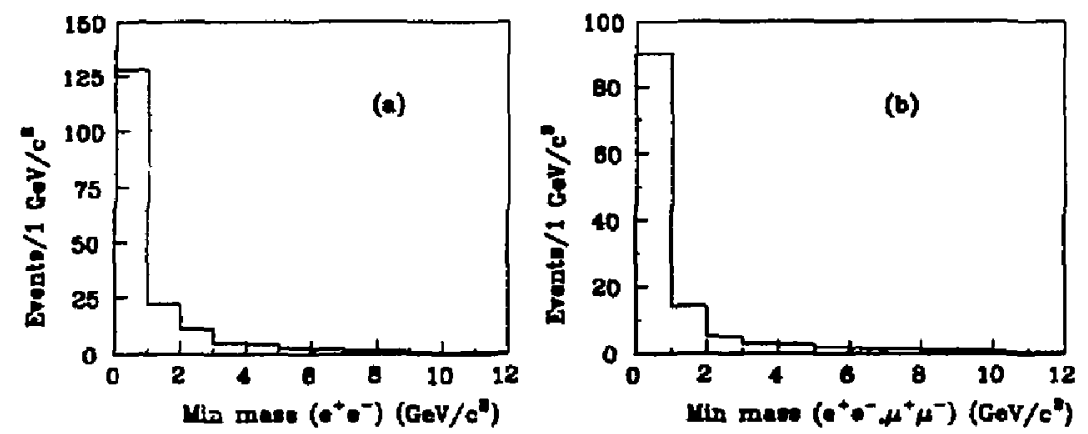

Figure 5.7. Lowest invariant mass distributions in $e^{+} e^{-} e^{+} e^{-}$events (a) and $e^{+} e^{-} \mu^{+} \mu^{-}$ events (b).

about $65 \%$ of the total cross section, but now the contribution from annihilation and conversion type of events is quite substantial, about $15 \%$ each.

It is interesting to note that the process $e^{+} e^{-} \mu^{+} \mu^{-}$, described by only 12 Feynmaj diagrams, has a cross section larger than that of $e^{+} e^{-} e^{+} e^{-}$, which is described by 36 Feynman diagrams. The reason is that for the $e^{+} e^{-} e^{+} e^{-}$set of diagrams, there are destructive interferences which contribute to a smaller cross section. To understand this point more clearly, we reran the $M C$ generator for the $e^{+} e^{-} \mu^{+} \mu^{-}$ process after seplacing the muon mass with that of the electron. This way, we calculated an $e^{+} e^{-} e^{+} e^{-}$cross section which corresponds only to the 12 diagrams of $e^{+} e^{-} \mu^{+} \mu^{-}$. We obtained a cross section of $(1.23 \pm 0.01) \mathrm{pb}$ in contrast to the cross section of $(0.88 \pm 0.01) \mathrm{pb}$ that was obtained when all $36 e^{+} e^{-} e^{+} e^{-}$diagrams were taken into account. This demonstrates the important role of the destructive interferences among these diagrams. Furthermore, this artificial 12-diagram cross section is slightly bigger than the $e^{+} e^{-} \mu^{+} \mu^{-}$cross section of $(1.14 \pm 0.01) \mathrm{pb}$. This 
result is not surprising, since it is easier for these processes to occur when the mass of the two leptons that come from the same photon is small.

\section{$5.1 .3 \mu^{+} \mu^{-} \mu^{+} \mu^{-}$}

Again, in this process, the angular and momentum distributions are similar to those observed for $e^{+} e^{-} e^{+} e^{-}$and $e^{+} e^{-} \mu^{+} \mu^{-}$final states.

When all four final-state muons are emitted in the angular interval $20^{\circ} \leq \theta \leq 160^{\circ}$ with respect to the electron beam direction, the total $\mu^{+} \mu^{-} \mu^{+} \mu^{-}$ cross section of generation is $(0.042 \pm 0.001)$ pb. The two-photon conversion contribution is about $70 \%$ of the total cross section. The rest of the cross section is made up from the annihilation cross section, and the interference between the two-photon conversior and the annihilation diagrams.

\subsection{Monte Carlo results}

\subsubsection{Mark II analysis of Monte Carlo signal events}

In this section, we show the characteristics of the MC generated signal events after they bave passed through a detector simulation program. Comparisons between Mark 11 data and MC predictions are shown in chapter 7. The generated events were passed through a full detector simulation, which included the effects of photon conversions, multiple Coulomb scattering, electromagnetic interactions in the calorimeters, dead wires and cell inefficiencies in the drift chamber, tube inefficiencies and hadron punchthrough in the muon system. The simulated events were passed through the same analysis code used for the real data analysis, and comparisons were made between theory and experiment (see chapter 7). All the 
distributions presented in this section have been normalized to $205 \mathrm{pb}^{-1}$, the Mark II detector's integrated luminosity.

The effect of the detector simulation on the track multiplicity distribution in the case of $e^{+} e^{-} e^{+} e^{-}, e^{+} e^{-} \mu^{+} \mu^{-}$and $\mu^{+} \mu^{-} \mu^{+} \mu^{-}$events is shown in fig. 5.11 (a), fig. 5.12 (a) and fig. 5.13 (a), respectively. The kinematic range of the generated events is larger than the detector coverage, resulting in a significant loss of tracks after simulation.
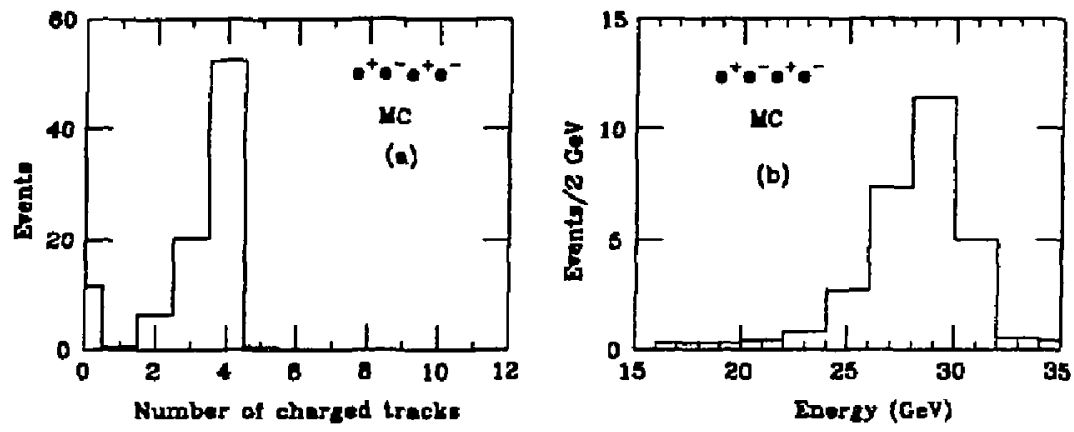

Figure 5.8. Track multiplicity (a) and energy (b) in Mark II $e^{+} e^{-} e^{+} e^{-}$MC events (see text). Events scaled to $205 \mathrm{pb}^{-1}$ integrated luminosity.

From now on, whenever we refer to the energy of an eveni we mean the total scalar momentum of the charged tracks times $c, \sum_{i=1,4} c\left|\vec{P}_{i}\right|$, except when stated otherwise. The energy distributions of events with 4 charged tracks with zero net charge, are shown in fig. 5.8 (b), 5.9 (b) and 5.10 (b), for $e^{+} e^{-} e^{+} e^{-}, e^{+} e^{-} \mu^{+} \mu^{-}$ and $\mu^{+} \mu^{-} \mu^{+} \mu^{-}$events, respectively. 

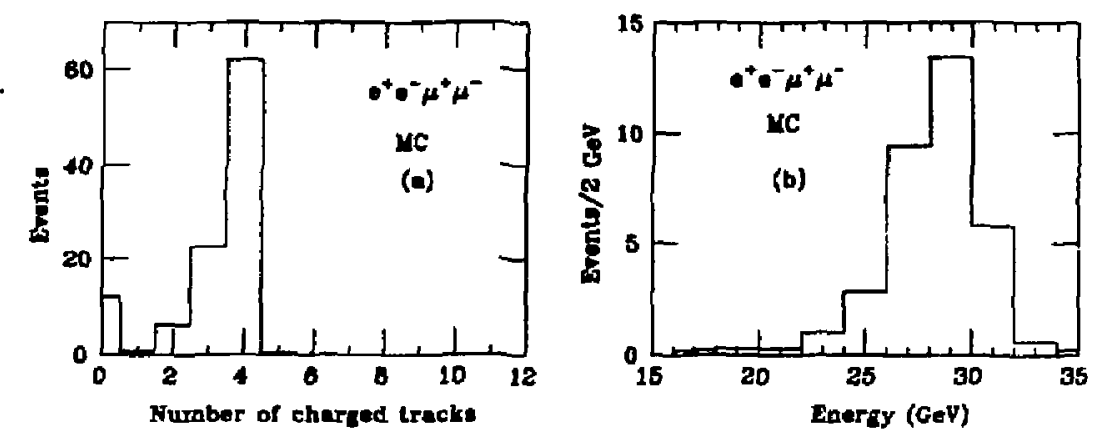

Figure 5.9. Track multiplicity (a) and energy (b) in Mark Il $e^{+} e^{-} \mu^{+} \mu^{-}$MC events (see text). Events scaled to $205 \mathrm{pb}^{-1}$ integrated luminosity.

The spread of the distributions around $29 \mathrm{GeV}$ has several sources. They include the effect of the drift chamber resolution and inefficiency, which alter the values of the generated momenta, as well as interactions, such as bremsstrahlung, which degrade the momenta of the scattered particles.
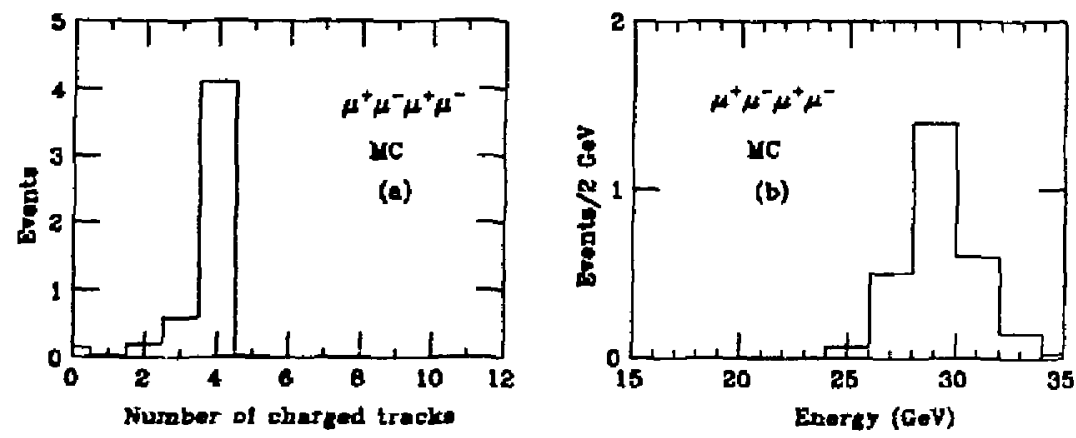

Figure 5.10. Track multiplicity (a) and energy (t) in Mark II $\mu^{+} \mu^{-} \mu^{+} \mu^{-}$MC events (sec text). Events scaled to $205 \mathrm{pb}^{-1}$ integrated luminosity. 


\subsubsection{HRS analysis of Monte Carlo signal events}

- The HRS detector simulation gives results that are qualitatively similar to those observed in the Mark II. Again, the generated events were passed through a full detector simulation. A subset* of the MC generated events, previously presented in table 5.1, was used in HRS. We summarize the MC integrated luminosities and event statistics for the three processes $e^{+} e^{-} e^{+} e^{-}, e^{+} e^{-} \mu^{+} \mu^{-}$and $\mu^{+} \mu^{-} \mu^{+} \mu^{-}$used in HRS in table 5.2 . The simulated events were passed through the same analysis code used for the real data analysis, and comparisons were made between theory and experiment. These comparisons will be discussed in chapter 8 . All the distributions presented in this section bave been normalized to $291 \mathrm{pb}^{-1}$, the HRS detector's integrated luminosity.

Table 5.2. Summary of MC event statistics and inlegrated luminosities for BRS.

\begin{tabular}{|c|c|c|c|}
\hline & $\begin{array}{c}\int L d t \\
\left(p b^{-1}\right)\end{array}$ & $\begin{array}{c}\text { Events } \\
\text { satisfying } \\
20^{\circ} \leq \theta \leq 160^{\circ}\end{array}$ & $\begin{array}{c}\text { Events satisfying } \\
P \geq 0.1 \mathrm{GeV} / \mathrm{c} \\
\mathrm{M}_{\text {pair }} \geq 0.5 \mathrm{GeV} / \mathrm{c}^{2}\end{array}$ \\
\hline$e^{+} e^{-} e^{+} e^{-}$ & 1598 & 1406 & 700 \\
\hline$e^{+} e^{-} \mu^{+} \mu^{-}$ & 1590 & 1796 & 800 \\
\hline$\mu^{+} \mu^{-} \mu^{+} \mu^{-}$ & 2059 & 85 & 50 \\
\hline
\end{tabular}

* The reason for only using a subset is twofold : a) the MC integrated luminomities used are still large compared to the HRS luminosity of $29 \mathrm{j} p b^{-1}$; b) Detector simulation is a very computer intensive procedure. 
The effect of the detector simulation on the track multiplicity distribution in each of the final states $e^{+} e^{-} e^{+} e^{-}, e^{+} e^{-} \mu^{+} \mu^{-}$and $\mu^{+} \mu^{-} \mu^{+} \mu^{-}$is shown in figure 5.11 (a), figure $5.12(\mathrm{a})$ and figure $5.13(\mathrm{a})$, respectively.

The energy distributions of events with 4 charged tracks with zero net charge are shown in fig. $5.11(\mathrm{~b}), 5.12(\mathrm{~b})$ and $5.13(\mathrm{~b})$, for $e^{+} e^{-} e^{+} e^{-}, e^{+} e^{-} \mu^{+} \mu^{-}$and $\mu^{+} \mu^{-} \mu^{+} \mu^{-}$events, respectively. These distributions are narrower than in the Mark II because of the better momentum resolution of HRS.
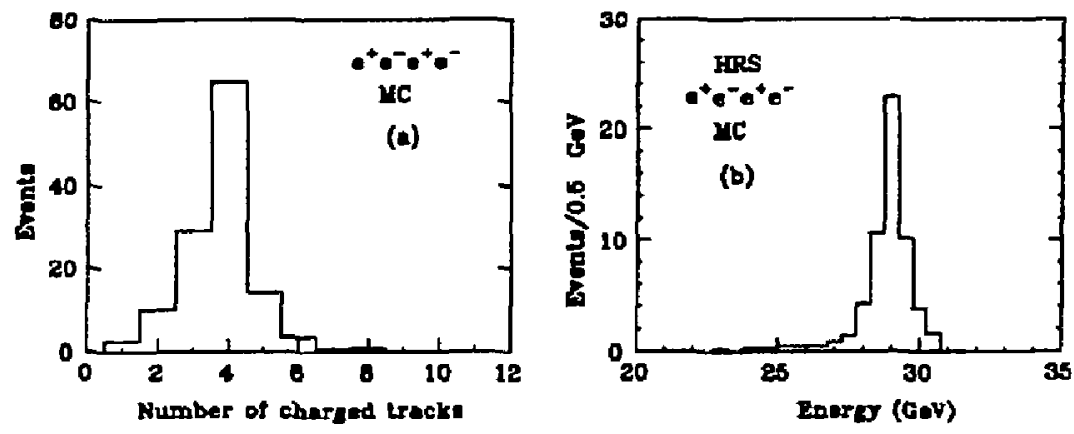

Figure 5.11. Track multiplicity (a) and energy (b) in HHS $e^{+} e^{-} e^{+} e^{-}$MC events (see Lext). Events scaled to $291 \mathrm{pb}^{-1}$ integrated luminosity. 

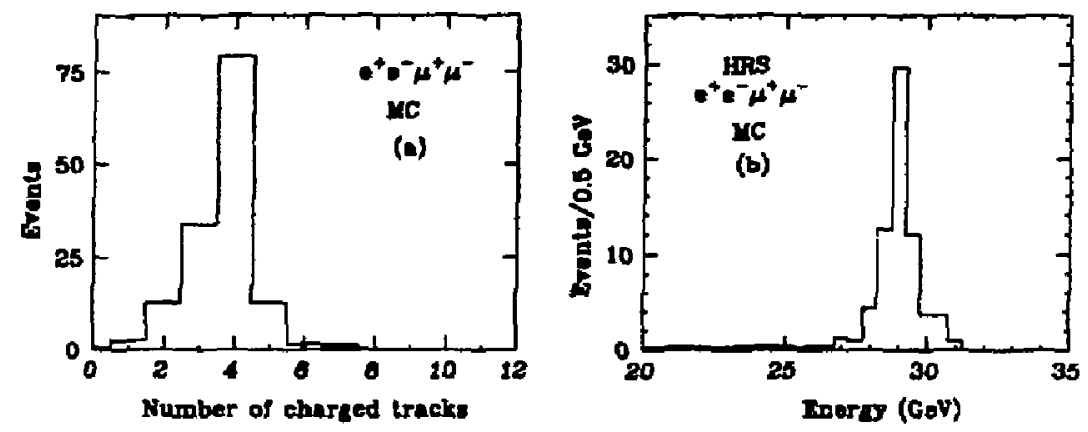

Figure 5.12. Track multiplicity (a) and energy (b) in HRS $e^{+} e^{-} \mu^{+} \mu^{-}$MC events (see text). Events scaled to $291 \mathrm{pb}^{-1}$ integrated luminosity.
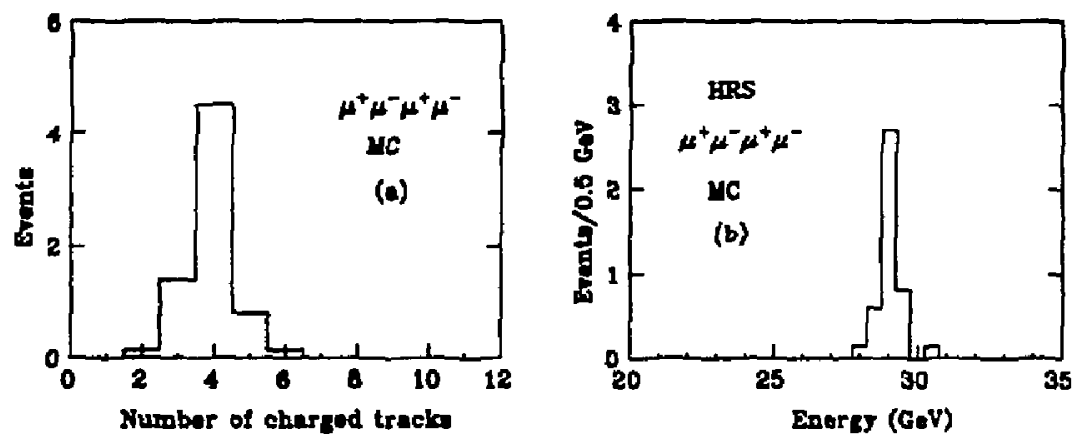

Figure 5.13. Track multiplicity (a) and energy (b) in HRS $\mu^{+} \mu^{-} \mu^{+} \mu^{-}$MC events (see text). Evente aculed to $291 \mathrm{pb}^{-1}$ integrated luminocity. 


\section{CHAPTER 6}

\section{MONTE CARLO BACKGROUND GENERATION AND ANALYSIS}

Two characteristic features of the four-lepton final states studied in this thesis $e^{+} e^{-} e^{+} e^{-}, e^{+} e^{-} \mu^{+} \mu^{-}, \mu^{+} \mu^{-} \mu^{+} \mu^{-}$, are :

- the events are composed of four charged tracks (electrons or muons) at large angles.

- the energy (total scalar momentum) distribution peaks at $29 \mathrm{GeV}$.

It is therefore useful to examine the multiplicity and energy distributions in processes which can fake the signal.

Reactions which can a priori contribute as background to the examined fourlepton processes are the following :

$$
\begin{aligned}
& e^{+} e^{-} \rightarrow e^{+} e^{-} T^{+} T^{-} \\
& e^{+} e^{-} \rightarrow e^{+} e^{-} h^{+} h^{-} \\
& e^{+} e^{-} \rightarrow \tau^{+} \tau^{-} \\
& e^{+} e^{-} \rightarrow q \bar{q} \rightarrow \text { hadrons } \\
& e^{+} e^{-} \rightarrow e^{+} e^{-} \gamma \gamma
\end{aligned}
$$


The most important source of background is $e^{+} e^{-} \rightarrow e^{+} e^{-} \tau^{+} \tau^{-}$, since both $\tau^{\prime}$ s may decay into a charged lepton (an electron or a muon) plus neutrinos. Events from this reaction were simulated using the Monte Carlo programs of Berends et al. (1-7) The kinematic cuts used for the generation of these $e^{+} e^{-} \tau^{+} \tau^{-} M C$ events were exactly the same as the ones used for the generation of $e^{+} e^{-} e^{+} e^{-}, e^{+} e^{-} \mu^{+} \mu^{-}$and $\mu^{+} \mu^{-} \mu^{+} \mu^{-} \mathrm{MC}$ events. We simply recall them here :

- The scattering angle of the final-state particles was required to be within the angular interval $20^{\circ} \leq \theta \leq 160^{\circ}$ with respect to the electron beam direction.

- All particles were required to have a momentum of at least $0.1 \mathrm{GeV} / \mathrm{c}$.

- All opposite-charge particle pair combinations were required to have an invariant mass of at least $0.5 \mathrm{GeV} / \mathrm{c}^{2}$.

The generated events corresponded to an integrated luminosity of $10055 \mathrm{pb}^{-1}$.

The background from $e^{+} e^{-} \rightarrow e^{+} e^{-} h^{+} h^{-}$, where $h$ is a hadron, was determined from simulated events, ${ }^{(31)}$ using the same Monte Carlo programs of Berends et al. for the generation of $e^{+} e^{-} \rightarrow e^{+} e^{-} q \bar{q}$ events. The kinematic cuts used for the generation of these $e^{+} e^{-} q \bar{q} \mathrm{MC}$ events were the following :

- The scattering angle of the final-state positron was required to be within the angular interval $40^{\circ} \leq \theta \leq 140^{\circ}$ with respect to the electron beam direction.

- The scattering angle of the final-state electron was required to be within the angular interval $0^{\circ} \leq \theta<40^{\circ}$ with respect to the electron beam direction.

These angular requirements are not unduly restrictive given the selection criteria on four-lepton final states discussed in chapter 7. 
- The final-state quarks $(q, \bar{q})$ could scatter at any angle with respect to the electron beam.

The Lund Monte Carlo code ${ }^{(32)}$ was used to fragment the quarks into hadrons. The events corresponded to an integrated luminosity of $5156 \mathrm{pb}^{-1}$.

The contribution of $e^{+} e^{-} \rightarrow{T^{+}}^{+}$as background comes when one $\tau$ decays into an electron or muon plus neutrinos and the other $\tau$ decays into charged and neutral pions plus a neutrino, where the pions simulate electrons or muons. The generated events ${ }^{(33)}$ corresponded to an integrated luminosity of $512 \mathrm{pb}^{-1}$.

The process $e^{+} e^{-} \rightarrow q \bar{q} \rightarrow$ hadrons may contribute either through the decay of a hadron to an electron or muon, or through the misidentification of a hadron as an electron or muon. The Lund Monte Carlo program with Luind and Peterson ${ }^{(34)}$ fragmentation methods was used ${ }^{(35)}$ to generate events corresponding to an integrated luminosity of $627 \mathrm{pb}^{-1}$.

Higher-order radiative Bhabha events, $e^{+} e^{-} \rightarrow e^{+} e^{-} \gamma \gamma$, can fake $e^{+} e^{-} e^{+} e^{-}$ events if the radiated photons are converted in the beam pipe, producing electrons. Due to the smallness of its cross section, this process is expected to contribute minimally to the background, at the high $Q^{2}$ regions examined in this study. A Monte Carlo gezerator ${ }^{(36)}$ was used to reproduce events corresponding to an integrated luminosity of $655 \mathrm{pb}^{-1}$.

For illustration, we present the behavjour of the MC generated background processes in the Mark II detector. The track multiplicity and energy (total scalar momentum) distributions of the simulated events for each of the background pro- 
cesses mentioned earlier, before any analysis cuts are applied, are shown in figures 6.1 : 6.5. In each of these figures, fig. (a) shows the number of tracks per event after detector simulation and fig. (b) presents the energy (total scalar momentum) distribution of events with four tracks only. Considering these distributions, as well as the corresponding ones for $e^{+} e^{-} e^{+} e^{-}, e^{+} e^{-} \mu^{+} \mu^{-}, \mu^{+} \mu^{-} \mu^{+} \mu^{-}$events (fig. 5.8 (b) 5.10 (b)), it is clear that the requirement of having only four charged tracks with an energy (total scalar momentum) above $20 \mathrm{GeV}$ will not only reject a large part of the background, but will also keep most of the signal. Other selection criteria, designed to reject specific types of background processes, have also been used. At this point, we would like to emphasize that these additional cuts, such as the zero-charge pair mass cut, greatly suppress the backgrounds remaining after the track multiplicity and energy cuts.

The background processes mentioned earlier, show similar general behaviour in the HRS detector. We comment now on the MC generated background event samples used in the HRS analysis.

The Monte Carlo generated $e^{+} e^{-} \tau^{+} \tau^{-}$events used in the HRS analysis are a subset of the sample used in Mark II. They correspond to a luminosity of $2530 \mathrm{pb}^{-1}$, which is substantially larger than the $291 p b^{-1}$ of HRS.

To study the contribution of $e^{+} e^{-} \rightarrow e^{+} e^{-h^{+} h^{-}}$we generated a new set of $e^{+} e^{-} q \bar{q} \mathrm{MC}$ events since the four-momenta files of the $e^{+} e^{-} q \bar{q} \mathrm{MC}$ events from Mark I] were not available. 

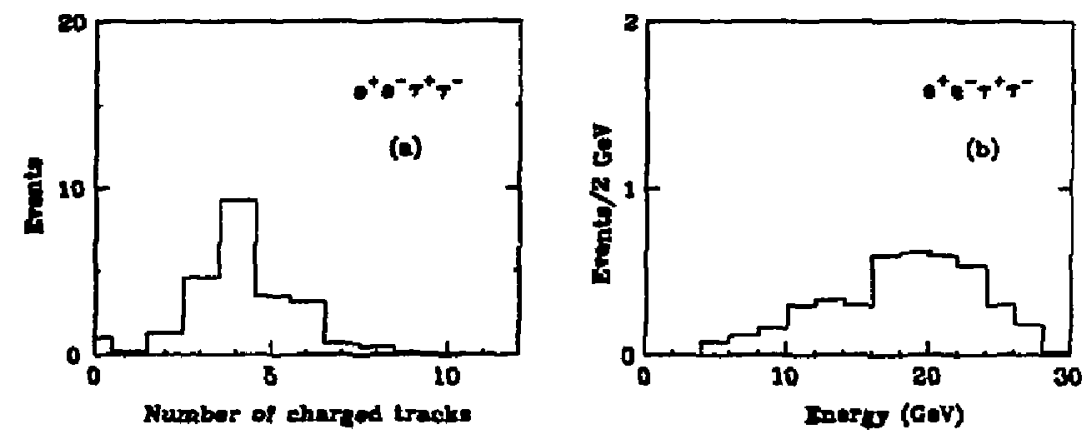

Figure 6.1. Track multiplicity diatribution (a) and energy distribution (b) ib $e^{+} e^{-T^{+}} \boldsymbol{r}^{-}$ MC events. Events scaled to $205 \mathrm{pb}^{-1}$ integtated Juminosity.
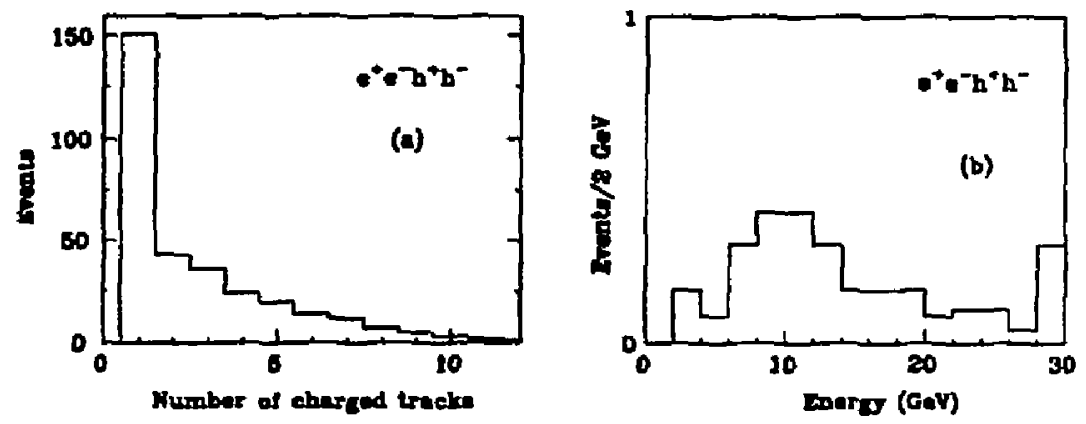

Figure 6.2. Truct multiplicity distribution (a) and energy distribution (b) in $e^{+} e^{-h^{+} h^{-}}$ MC everts. Events acaled to $205 \mathrm{pb}^{-1}$ integrated luminonity. 

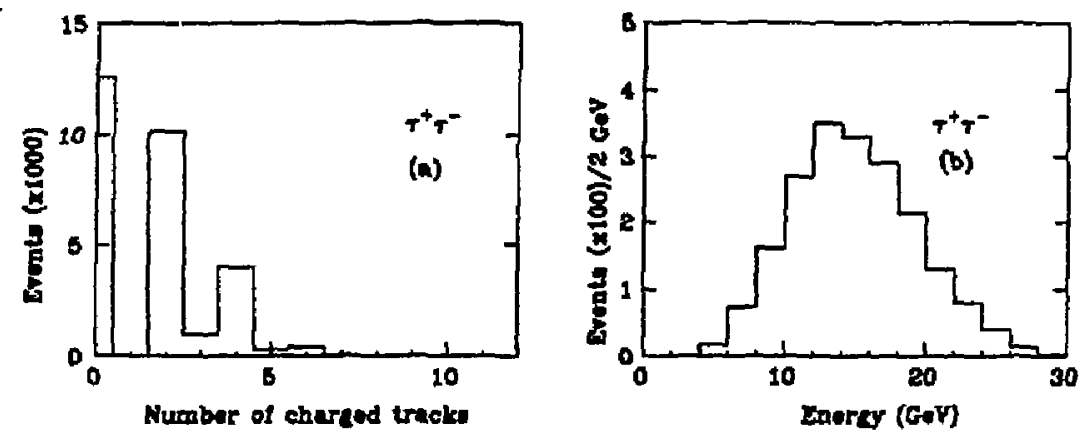

Figure 6.3. Track multiplicity distribution (a) and energy diatribution (b) in $\boldsymbol{\tau}^{+} \boldsymbol{\tau}^{-} \mathbf{M C}$ events. Events acaled to $205 \mathrm{pb}^{-1}$ integrated luminonity.
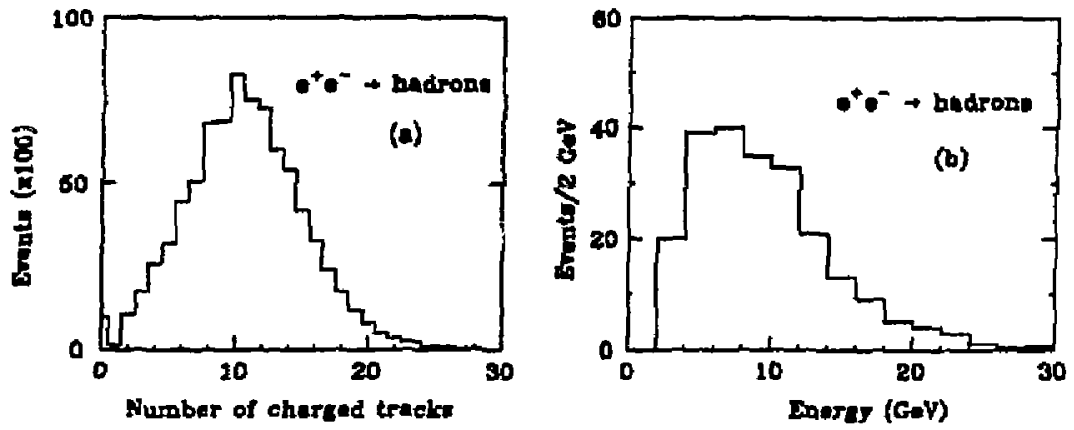

Figure 6.4. Track multiplieity distribution (a) and energy distribution (b) in MC hadronic events. Events scaled to $205 \mathrm{pd}^{-2}$ integraled luminosity. 

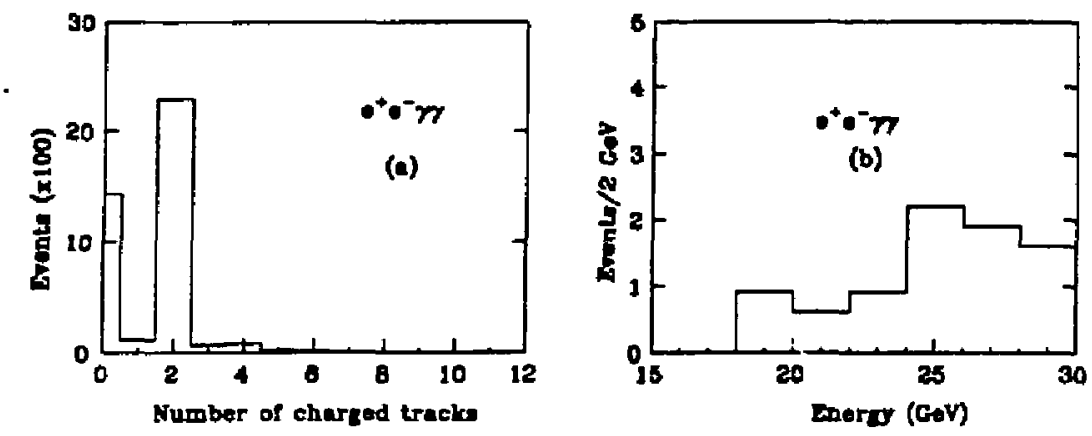

Figure 6.5. Track multiplicity distribution (a) and energy distribution (b) in $e^{+} t^{-} \gamma$ MC events. Events scaled to $205 \mathrm{pb}^{-1}$ integrated luminosity.

The kinematic cuts used for the generation of the $e^{+} e^{-} q \bar{q} \mathrm{MC}$ events for HRS were identical to the ones used for the gencration of $e^{+} e^{-} e^{+} e^{-}, e^{+} e^{-} \mu^{+} \mu^{-}$, $\mu^{+} \mu^{-} \mu^{+} \mu^{-}$ard $e^{+} e^{-} \tau^{+} \tau^{-}$final states. The Lund Monte Carlo code was used to fragment the quarks into hadrons. The generated events corresponded to an integrated luminosity of $933 \mathrm{pb}^{-1}$. The contribution of $e^{+} e^{-} \rightarrow \tau^{+} \tau^{-}$was studied by using $\tau^{+} \tau^{-} M C$ generated events, ${ }^{(37)}$ corresponding to an integrated luminosity of $294 p^{-1}$. Finally, the sample of events of the type $e^{+} e^{-} \rightarrow q \bar{q} \rightarrow$ hadrons corresponded to the integrated luminosity of $185 \mathrm{pb}^{-1}$.

In the next two chapters we shall present the complete list of the selection criteria for four-lepton final states, in the Mark II and HRS experiments. 


\section{CHAPTER 7}

\section{MARK II DATA ANALYSIS}

In this chapter, the experimental data from the Mark II detector are compared with the QED calculations from chapters 4,5 and 6 . The first section presents the event selection and the analysis of high $Q^{2}$ data events. A comparison of these candidate four-lepton events with the MC data and background processes follows. Finally, we briefly discuss the detection efficiencies for the three processes $e^{+} e^{-} e^{+} e^{-}$, $e^{+} e^{-} \mu^{+} \mu^{-}, \mu^{+} \mu^{-} \mu^{+} \mu^{-}$. For the sake of brevity, we shall denote electrons and positrons by the collective term 'electrons', and similarly for muons.

\subsection{Event selection and analysis}

The four-lepton event selection proceeded in two stages. The original data sample $\left(205 \mathrm{pb}^{-1}\right)$ was reduced to 6532 events by a first program filter. The filter selected events with loose enough criteria so as to be efficient for four-body QED final states. The selection criteria used were the following :

All events must have at least 4 'good' charged tracks. In events with only 4 'good' charged tracks, the net total charge should be zero. A 'good' track satisfies the following criteria : 
1. momentum $P>0.1 \mathrm{GeV} / \mathrm{c}$

2. pass close to the interaction point $(2 \leq 0.1 \mathrm{~m}, \mathrm{r} \leq 0.05 \mathrm{~m})$

3. bit at least a total of 5 layers in the drift chamber

4. have a $\chi^{2}$ per degree of freedom $\leq 20$.

The 6532 events which survived the above cuts were passed through the second and final analysis filter. The second filter applied stricter acceptance criteria, designed to select four-lepton final states while eliminating background events.

The event selection and event identification requirements were crystallized after a study of the background processes was cosinpleted. We found that, for the high $Q^{2}$ (large pair mass) final states that interest us, barkground problems are minimal. Lepton identification and pair mass cuts proved to be powerful tools for eliminating backgrounds.

We present two analyses, (A) and (B), which differ in the requirements placed on the invariant mass of any zero-charge pair of tracks. Both cases were examined in view of our goal of testing QED at small distances (or high $Q^{2}$ ).

In case (A), each event was required to satisfy the following criteria :

1. Four good charged tracks with zero net total charge. A good track has a momentum of at least $0.5 \mathrm{GeV} / \mathrm{c}$, passes sufficiently close to the interaction point $(s \leq 0.05 \mathrm{~m}, 2 \leq 0.1 \mathrm{~m})$, through at least 6 drift chamber and 3 vertex chamber layers, and has a $\chi^{2}$ per degree of freedom of at most 10 .

2. At least two tracks should be in the barrel of the detector, $|\cos \theta|<\cos 45^{\circ}$. This condition was imposed because the Mark II trigger required at least two 
tracks in this angular region in the absence of a significant calorimeter signal. The other tracks could be at any other large angle above $28^{\circ}$ with respect to the beam direction.

3. The energy of an event, which was defined as the scalar sum of the charged particle momenta times $c, \sum_{i=1,4} c\left|P_{i}\right|$, should be at least 20 and not more than $40 \mathrm{GeV}$. Four-lepton final state processes may have a $\sum_{i=1,4} c\left|P_{i}\right|$ different from $29 \mathrm{GeV}$, the c.m. energy, due to initial or final-state radiation. Collinear initial-state radiation is not visible in the detector, but affects the observed cross section. Final-state radiation is in principle visible in the detector. In appendix A we present the method used to estimate the effect of collinear initial-state radiation on the measured cross sections, and the results obtained. The energy distribution of the data before this cut is applied is shown in fig. 7.1. Note that the tail above $29 \mathrm{GeV}$ arises from imprecise momentum measurements of tracks, due to resolution effects.

4. All pairs of tracks with zero net charge must have an invarjant mass of at least $1 \mathrm{GeV} / \mathrm{c}^{2}$. This cut belps reduce the background coming from hadronic resonance production, as well as the background from single-photon conversion to an $e^{4} e^{-}$pair. The effect of this cut on the data sample is shown in fig. 7.2 , where the invariant mass distribution of the data is presented in the form it had before this cut was applied. The invariant masses shown range from 0 to $2 \mathrm{GeV} / \mathrm{c}^{2}$. From a physical point of view, it also ensures that this analysis is a test of QED at small distances (or high $Q^{2}$ ). 


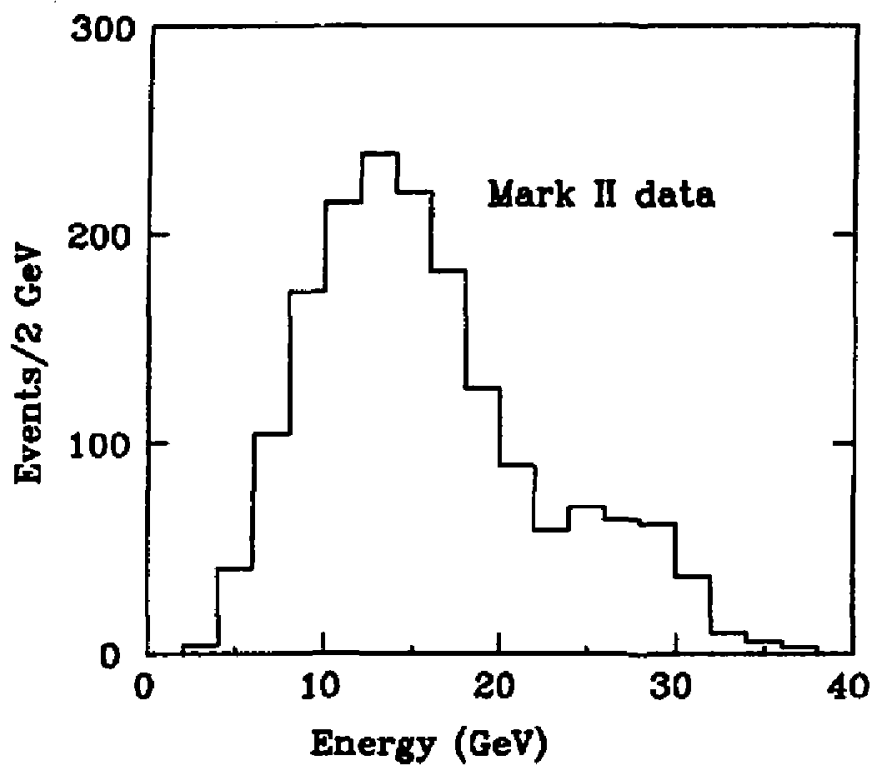

Figure 7.1. Mark II data energy diatribution before cut (3) is applied

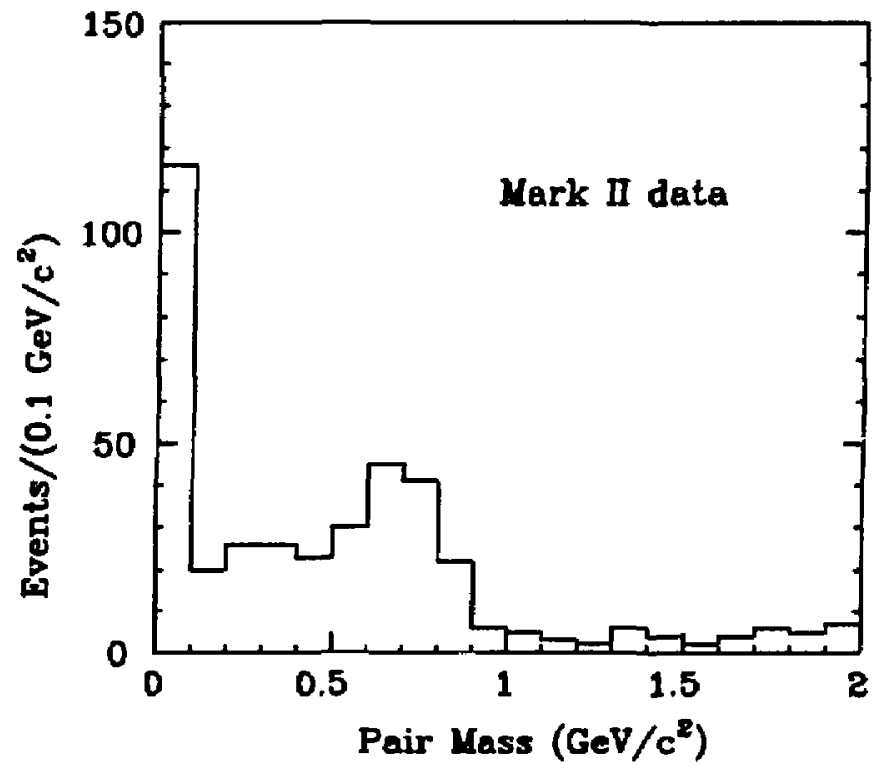

Figure 7.2. Mark II dala invarianl mas distribution before cut (4) is applied 
In case (A), the effect of each cut on the original data sample is given in table 7.1 .

Table 7.1. Effect of kinematical cuto on Mark II data in case (A).

\begin{tabular}{|l|c|c|c|c|}
\hline & Cut 1 & Cut 2 & Cut 3 & Cut 4 \\
\hline \# of Events & 1726 & 1699 & 395 & 38 \\
\hline
\end{tabular}

Case (B) was basically a check of the stability of our results under kinematical cuts less severe than the pair mass cut of $1 \mathrm{GeV} / \mathrm{c}^{2}$. The cuts used in case (B) were identical to those of case (A), except for cut 4 , which in case (B) required that all pairs of tracks have an invariant mass of at least $0.6 \mathrm{GeV} / \mathrm{c}^{2}$. An additional kinematical cut, used in case (B) to reduce background coming from $\tau$ decays, did not affect at all the results of case (A). This additional cut demanded that the invariant mass of any three tracks be at least $1.6 \mathrm{GeV} / \mathrm{c}^{2}$. The effect of this cut on the data sample is shown in fig. 7.3. We present there the distribution of the smallest mass that can be obtained from combinations of any three tracks in an event, in the form it had before the application of the cut on the data. The masses shown range from 0 to $4 \mathrm{GeV} / \mathrm{c}^{2}$.

In case (B), the effect of each cut on the original data sample is given in table 7.2. In this table, cut 5 is the requirement that the effective mass of any three charged tracks be equal to at least $1.6 \mathrm{GeV} / \mathrm{c}^{2}$.

We identify electrons and muons by examining the momentum $\mathrm{P}$ measured by the drift chambers, the energy $E$ deposited in the calorimeters and the signal from the muon chambers. This energy $E$ of a particle should not be confused with the 


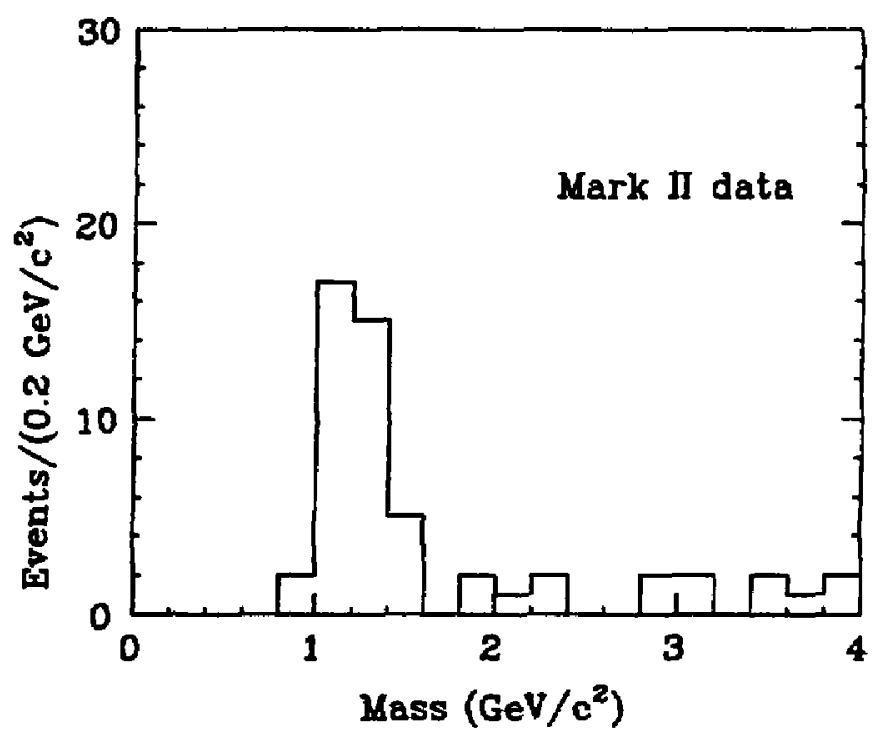

Figure 7.3. Distribution of the smallest among the masses obtsined from: any 3 tracks, as it was before the application of cut (5) on the Mark II data, in cage (B).

Table 7.2. Effect of kinematical cuts on Mark II data in case (B).

\begin{tabular}{|c|c|c|c|c|c|}
\hline & Cut 1 & Cut 2 & Cut 3 & Cut 4 & Cut 5 \\
\hline \# of Events & 1726 & 1699 & 395 & 104 & 65 \\
\hline
\end{tabular}

energy of an event, defined earlier in this section. We remind the reader about the parts of the Mark II detector, previously described in chapter 2, which were used for lepton identification. The Mark II detector bad a central drift chamber which provided us with momentum measurement of tracks emerging at angles above $28^{\circ}$. In the central region there was a liquid argon electromagnetic calorimeter (LA) that measured the energies deposited by particles with $|\cos \theta|<0.7$ with respect to the electron beam direction. The energies of particles with $0.76 \leq|\cos \theta| \leq 0.85$ were measured by two end cap shower counters (EC) located symmetrically on either side 
of the interaction point. In addition to the calorimeter and shower counters, there was a dedicated muon system covering about $55 \%$ of the solid angle.

In general, the ratio $\mathrm{E} / \mathrm{P}$ gives a reliable tool for identifying electrons and muons. In a perfect detector with excellent resolution and without cracks, E/P must equal one for electrons, whereas for muons, which are non-showering particles, E/P must be very small. In real detectors though, tracks with $\mathrm{E} / \mathrm{P}>0.5$ are usually identified as electrons, while tracks with $\mathrm{E} / \mathrm{P}<0.5$ are identified as muons. Additional requirements on the shower shapes of the particles traversing an electromagnetic calorimeter differentiate electrons from hadrons with $\mathrm{E} / \mathrm{P}>\mathbf{0 . 5}$. In the Mark II detector, the presence of a system of hadron absorber/proportional wire chamber allows a fairly strict muon identification by eliminating hadrons which could be passed off as muons.

Having all the above comments in mind, we imposed the following identification requirements :

There must be at least 3 identified leptons in an event, of 2 identified leptons of the same charge sign. An jdentified lepton must have a momentum of at least $1 \mathrm{GeV} / \mathrm{c}$ in order to minimize hadronic misidentification. The presence of low backgrounds allowed us to use rather loose identification criteria for leptons. The identification criteria for leptons are the following:

1. An electron candidate in the LA calorimeter must satisfy the shower development criteria that are characteristic of an electromagnetic shower. Also the energy $\mathbf{E}$ measured in the LA calorimeter and the momentum $\mathrm{P}$ measured 
with the drift chamber must be compatible. ${ }^{(3 a)}$ This requirement is essentially equivalent to $\mathrm{E} / \mathrm{P}>0.6$. In the $\mathrm{EC}$ shower counter, a candidate electron must have a momentum of at least $3 \mathrm{GeV} / \mathrm{c}$ and, $\mathrm{E} / \mathrm{P}>0.5$ (see appendix $\mathrm{B}$ ).

2. A muon candidate is required to leave hits in the layers of the muon system, in accordance with its expected range and track extrapolation error. A real muon would have to scatter in position or momentum by more than three standard deviations to avoid being detected, when we use this criterion. ${ }^{(38)}$

When only 3 leptons are identified in an event, the fourth is assumed to conserve lepton flavor. In events with 2 identified leptons of the same sign, the identities of the two other tracks are fixed by the assumption of lepton flavor conservation.

The E/P distribution of the data events which passed all kinematical requirements is shown in fig. 7.4.

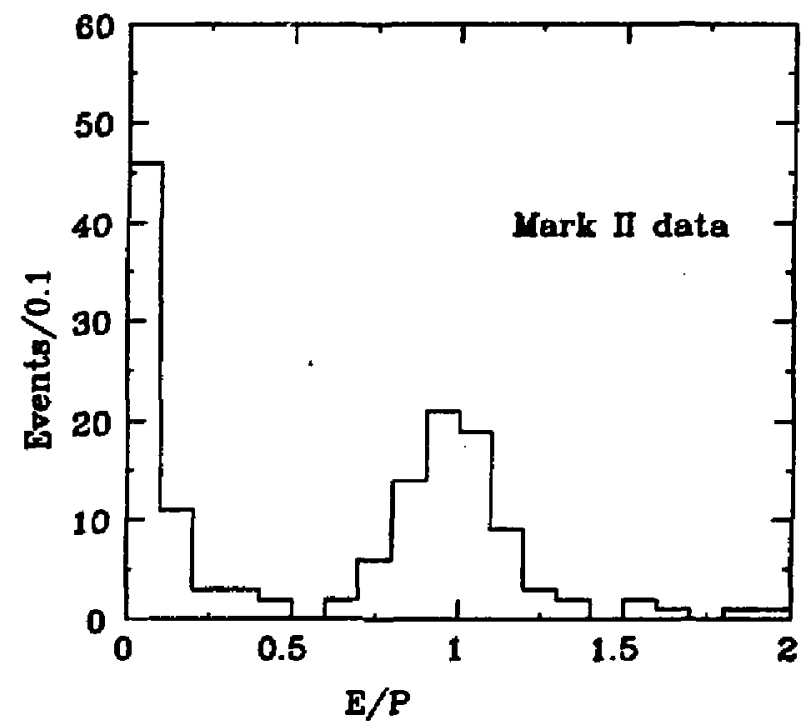

Figure 7.4. E/P diatribution of the Mark II four-lepton candidate events. 
Of the 38 events which passed all kinematical cuts of case (A), 21 events were identified. Ten were identified as $e^{+} e^{-} e^{+} e^{-}$, ten as $e^{+} e^{-} \mu^{+} \mu^{-}$and one as $\mu^{+} \dot{\mu}^{-} \mu^{+} \mu^{-}$. Of the $10 e^{+} e^{-} e^{+} e^{-}$events, 4 had all four leptons identified as electrons, 5 had three, and 1 event had two leptons of the same charge sign identified as electrons. Of the $10 e^{+} e^{-} \mu^{+} \mu^{-}$events, 4 had all four leptons identified, 5 had three, and 1 event had two leptons of the same charge sign identified. The $\mu^{+} \mu^{-} \mu^{+} \mu^{-}$ event had three leptons jdentified as muons. In case a lepton is identified by the assumption of lepton number conservation, we make sure that the event identification is appropriate, by looking at the event display and available tracking information. In other words, we check that the assignment inferred from the assumption of lepton number conservation is consistent with that made by our identification algorithm for those tracks that are not explicitly identified as leptons. Therè were no such discrepancies in the Mark II data.

Events which could not be identified as containing four leptons had tracks which :

i) were outside the calorimeters' fiducial volume, so that their energy deposition could not be tested; or

ii) were in the ralorimeters' fiducial volume but failed the lepton identification criteria; or

iij) missed the muon system, thus not giving any information about candidate muon tracks.

The list of the 38 events which passed all kinematical requirements in case 
(A), some of their properties and their identification assignments are given in appendix $\mathbf{C}$.

Of the 65 events which passed all kinematical cuts of case (B), 28 events were identified : 14 as $e^{+} e^{-} e^{+} e^{-}, 13$ as $e^{+} e^{-} \mu^{+} \mu^{-}$and 1 as $\mu^{+} \mu^{-} \mu^{+} \mu^{-}$. Of the 14 $e^{+} e^{-} e^{+} e^{-}$events, 5 had all fout leptons identified as electrons, 8 had three, and 1 event had two leptons of the same charge sign identified as electrons. Of the 13 $e^{+} e^{-} \mu^{+} \mu^{-}$events, 4 had all four leptons identified, 8 had three, and 1 event had two leptons of the same charge sign identified. The $\mu^{+} \mu^{-} \mu^{+} \mu^{-}$event had three leptons identified as muons. Thirty-seven events did not satisfy the identification criteria. In this case, the greater proportion of events failing the lepton identification requirements is consistent with an increase in four-prong events from sources other than four-lepton production when the pair mass cut is relaxed to $0.6 \mathrm{GeV} / \mathrm{c}^{2}$.

\subsection{Monte Carlo event selection and comparison with data}

The QED predictions were obtained by analyzing the Monte Carlo generated and detector simulated signal and background events, presented in chapters 5 and 6 , with the same programs used for the analysis of the real data events. As mentioned in chapter 5, all generation limits in angle, energy and invariant mass extended a few standard deviations beyond the final acceptance criteria.

We now present the results of Monte Carlo event selection and background rejection in both cases (A) and (B). Table 7.3 for case (A), and table 7.4 for case (B), list the effect of the analysis requirements imposed on the Monte Carlo generated signal and background processes. For each process, the number of events surviving 
each cut are listed under the corresponding column, while the initial number of events is given in the second column. The luminosity of the Monte Carlo event samples greatly exceeded the actual Mark II luminosity of $205 \mathrm{pb}^{-1}$, ws can be seen in tables 7.3 and 7.4 .

The number of expected signal events from Monte Carlo, when scaled to a luminosity of $205 \mathrm{pb}^{-1}$, was $10.9 \pm 0.7,9.6 \pm 0.8$ and $0.76 \pm 0.07$ for $e^{+} e^{-} e^{+} e^{-}, e^{+} e^{-} \mu^{+} \mu^{-}$ and $\mu^{+} \mu^{-} \mu^{+} \mu^{-}$respectively, while 10,10 and 1 events were observed in case (A). In case (B), the expected number of signal events was $14.0 \pm 0.8,12.0 \pm 0.9$ and $1.03 \pm 0.09$ for $e^{+} e^{-} e^{+} e^{-}, e^{+} e^{-} \mu^{+} \mu^{-}$and $\mu^{+} \mu^{-} \mu^{+} \mu^{-}$respectively, while 14,13 and 1 events were observed. The errors attached to the predicted values are statistical only.

Table 7.3. Effect of analysis cuts on Monte Carlo aignal and background events in case (A). The integrated luminosity corresponding to each procen is given in $p^{-1}$.

\begin{tabular}{|c|c|c|c|c|c|c|c|}
\hline case $(A)$ & $\int L d t$ & \# Events & Cut 1 & Cut 2 & Cut 3 & Cut 4 & ID \\
\hline$e^{+} e^{-} \rightarrow e^{+} e^{-} e^{+} e^{-}$ & 6005 & 2686 & 890 & 743 & 726 & 460 & 360 \\
\hline$e^{+} e^{-} \rightarrow e^{+} e^{-} \mu^{+} \mu^{-}$ & 3682 & 1853 & 638 & 517 & 506 & 319 & 194 \\
\hline$e^{+} e^{-} \rightarrow \mu^{+} \mu^{-} \mu^{+} \mu^{-}$ & 28343 & 688 & 378 & 338 & 337 & 205 & 118 \\
\hline$e^{+} e^{-} \rightarrow e^{+} e^{-} \tau^{+} \tau^{-}$ & 10055 & 1196 & 204 & 177 & 73 & 50 & 14 \\
\hline$e^{+} e^{-} \rightarrow e^{+} e^{-} h^{+} h^{-}$ & 5156 & 7940 & 68 & 44 & 11 & 6 & 0 \\
\hline$e^{+} e^{-} \rightarrow e^{+} e^{-} \gamma \gamma$ & 655 & 12736 & 31 & 29 & 26 & 0 & 0 \\
\hline$e^{+} e^{-} \rightarrow q \bar{q} \rightarrow h a d r o n s$ & 627 & 250875 & 697 & 573 & 22 & 5 & 0 \\
\hline$e^{+} e^{-} \rightarrow \tau^{+} \tau^{-}$ & 512 & 70885 & 5000 & 4544 & 605 & 3 & 0 \\
\hline
\end{tabular}


Table 7.4. Effect of analysis cuts on Monte Carlo aigal and background events in case (B). The integrated luminosity corresponding to each process is given in $p^{-1}$.

\begin{tabular}{|c|c|c|c|c|c|c|c|c|}
\hline case (B) & $\int L d t$ & \# Events & Cut 1 & Cut 2 & Cut 3 & Cut 4 & Cut 5 & ID \\
\hline$e^{+} e^{-} \rightarrow e^{+} e^{-} e^{+} e^{-}$ & 6005 & 2686 & 890 & 743 & 726 & 646 & 638 & 463 \\
\hline$e^{+} e^{-} \rightarrow e^{+} e^{-} \mu^{+} \mu^{-}$ & 3682 & 1853 & 638 & 517 & 506 & 442 & 442 & 243 \\
\hline$e^{+} e^{-} \rightarrow \mu^{+} \mu^{-} \mu^{+} \mu^{-}$ & 28343 & 688 & 378 & 338 & 337 & 284 & 281 & 160 \\
\hline$e^{+} e^{-} \rightarrow e^{+} e^{-} \tau^{+} \tau^{-}$ & 10055 & 1196 & 204 & 177 & 73 & 64 & 64 & 15 \\
\hline$e^{+} e^{-} \rightarrow e^{+} e^{-} h^{+} h^{-}$ & 5156 & 7940 & 68 & 44 & 11 & 10 & 10 & 0 \\
\hline$e^{+} e^{-} \rightarrow e^{+} e^{-} \gamma \gamma$ & 655 & 12736 & 31 & 29 & 26 & 0 & 0 & 0 \\
\hline$e^{+} e^{-} \rightarrow q \bar{q} \rightarrow$ hadrons & 627 & 250875 & 697 & 573 & 22 & 9 & 9 & 0 \\
\hline$e^{+} e^{-} \rightarrow \tau^{+} \tau^{-}$ & 512 & 70885 & 5000 & 4544 & 605 & 197 & 9 & 0 \\
\hline
\end{tabular}

Among the observed identified events we searched for photons with an energy greater than $1 \mathrm{GeV}$, and with an angle greater than $10^{\circ}$ from the nearest track. There was only one $e^{+} e^{-} \mu^{+} \mu^{-}$event with such a photon. It had $1.5 \mathrm{GeV}$ of energy and made an angle of $14.6^{\circ}$ with the nearest track. This event cannot be compared separately with theory, since there is no complete QED calculation ${ }^{(30)}$ for the radjative corrections to order $\alpha^{4}$.

The emission of a real or virtual photon from the electron or positron before they annihilate can affect the observed cross section. We therefore attempted to estimate the correction to the four-lepton cross section for initial-state radiation. In our estimate we made use of the factorization of the infrared contributions, and of the strong peaking of the photon cross section in directions parallel to the motion of the charged particles. We used the probability function of Kuraev and Fadin (40) 
to describe the emission of photons. We generated four-electron events, which we :-qulated and passed through the same analysis programs as the real data, at various center-of-mass energies below $29 \mathrm{GeV}$. All appropriate kinematical transformations between the center of mass and the laboratory frames were taken into account. We found that the ratio of the cross section of $e^{+} e^{-} \rightarrow e^{+} e^{-} e^{+} e^{-}$, corrected for initial state radiation, over the observed $e^{+} e^{-} \rightarrow e^{+} e^{-} e^{+} e^{-}$cross section is $0.98 \pm 0.08$, where the error is due to limited MC statistics (see appendix A).

The predicted numbers of signal and background events that would pass all cuts at an integrated luminosity of $205 \mathrm{pb}^{-1}$ are shown in table 7.5 and table 7.6 for cases (A) and (B), respectively. The 95\% CL upper limits are given in background processes where zero events passed the identification cuts.

The number of data events is presented without background subtraction. The expected numbers of signal and background events have been corrected track by track for lepton identification efficiency of $0.97 \pm 0.02$ for electrons and muons, (41) and for initial-state radiation. In particular, the number of identified events with $n$ tracks identified as leptons is multiplied by $0.97^{n}$. So, for events witi 4,3 and 2 identified leptons, the lepton identification correction factor is $0.88,0.91$ and 0.94, respectively. The breakdown of Monte Carlo events in categories with 4, 3 and 2 identified leptons is shown in table 7.7 and table 7.8 for cases (A) and (B), respectively. The following numerical example shows bow these factors are used to calculate the final numbers of expected events shown in tables 7.5 and 7.6. In order to obtain the total number of expected $e^{+} e^{-} e^{+} e^{-}$events for case (A), we first 
multiply the numbers 125,208 and 27 corresponding to the categories $l l l,\|l x\| x$, (see table 7.7 ), with the appropriate factors $0.88,0.91$, and 0.94 , respectively. We then multiply by 0.98 to correct for initial state radiation, and we scale down the result from $6005 b^{-1}$ to $205 p b^{-1}$, obtaining the final answer shown in table 7.5 :

$$
10.9=\frac{205}{8005} \times 0.98 \times(125 \times 0.88+208 \times 0.91+27 \times 0.94)
$$

The errors attached to the predicted values (signal and background) are statis. tical, whereas the errors in the total number of expected events are statistical (first error) and systematic (second error). The systematic error consists of the error of the relative particle jdentification efficiencies in the data and the Monte Carlo simulations ( $2 \%)$, and the systematic uncertainty in the integrated luminosity of the data ( $1.5 \%)$, added in quadrature.

We tested the sensitivity of our results to the identification cuts used. We define $\mathbf{R}_{\text {tight }}\left(\mathbf{R}_{\text {loose }}\right)$ as the ratio of the number of observed data from all three processes $\left(e^{+} e^{-} e^{+} e^{-}, e^{+} e^{-} \mu^{+} \mu^{-}, \mu^{+} \mu^{-} \mu^{+} \mu^{-}\right)$over the number of corresponding predicted events, when tight(loose) identification cuts and the kinematical cuts of case (B) have been used. We define as loose identification cuts the ones used in the analyses presented previously, whereas tight identification cuts are defined as follows:

- An electron candidate in the LA calorimeter is tested with more restrictive criteria. This time, the electron identification requirement is essentially equivalent to $E / P>0.8$. Energy information from the $E C$ shower counter is not used. 
Table 7.5. Number of observed (no background subtraction) and expected signal and background events for $e^{+} e^{-} e^{+} e^{-}, \quad e^{+} e^{-} \mu^{+} \mu^{-}$and $\mu^{+} \mu^{-} \mu^{+} \mu^{-}$in case (A) (pair masses $\geq 1 \mathrm{GeV} / \mathrm{c}^{2}$ ). Shown upper limits are at $95 \% \mathrm{CL}$.

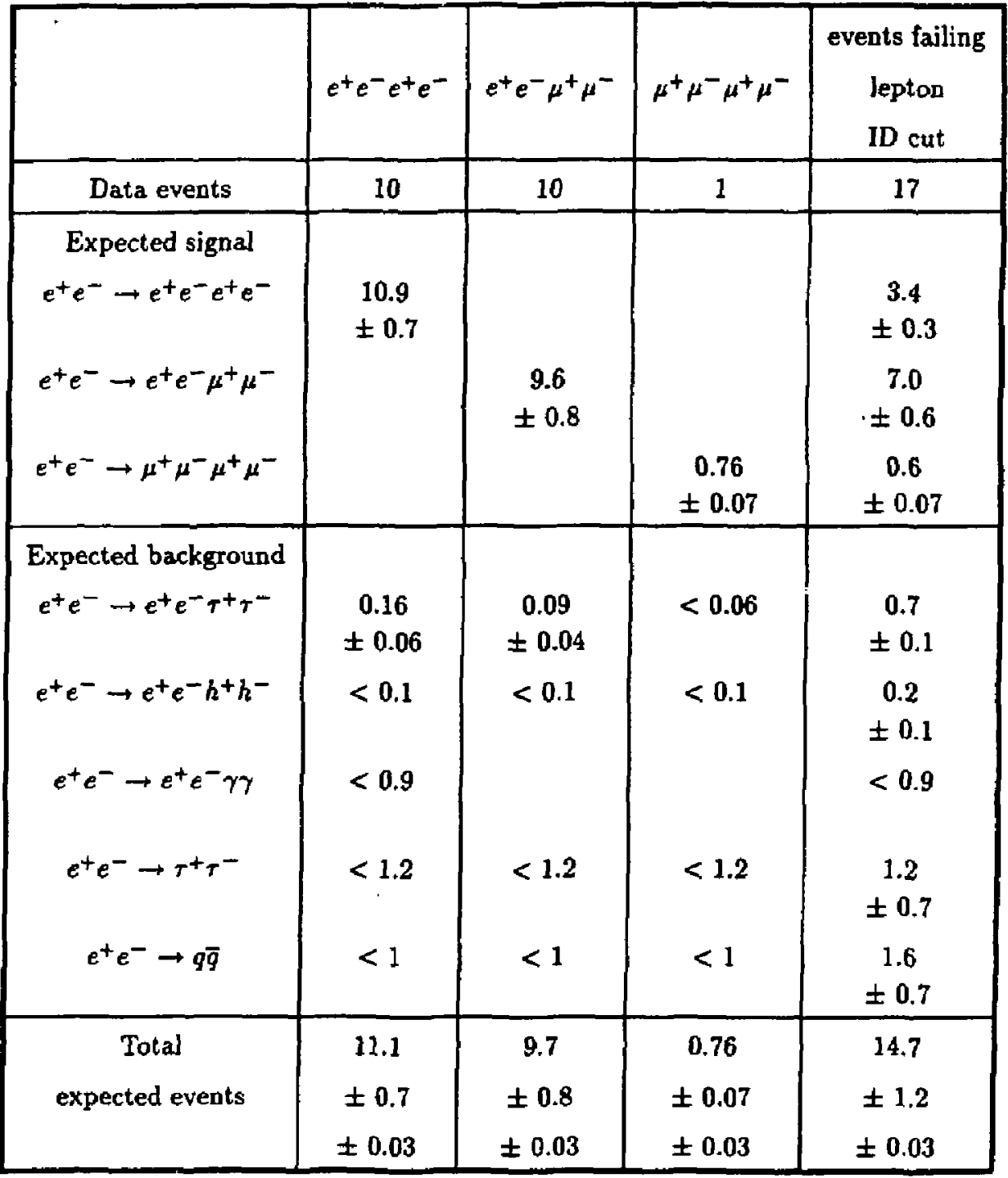


Table 7.6. Number of observed (no background oubtraction) and expected nignal and background events for $e^{+} e^{-} e^{+} e^{-}, e^{+} e^{-} \mu^{+} \mu^{-}$and $\mu^{+} \mu^{-} \mu^{+} \mu^{-}$in case (B) (pair masses $\geq 0.6 \mathrm{GeV} / \mathrm{c}^{2}$ ). Shown upper limits are at $95 \% \mathrm{CL}$.

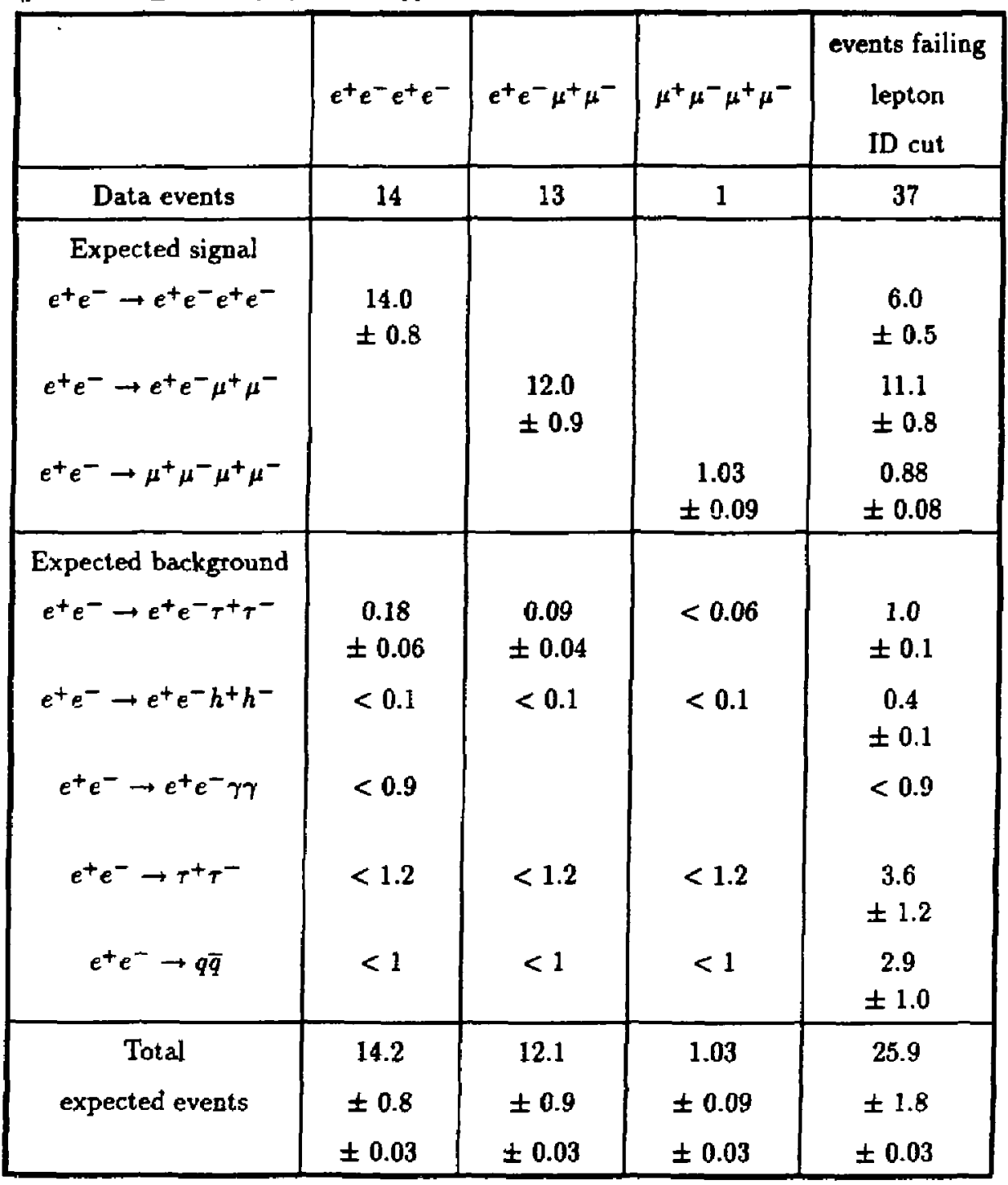


- A muon candidate is required to leave hits in either the first three or all four layers of the muon system, consistent with its expected range and track exsrapolation error.

We observed a total of 28 events from the three processes when loose identifcation cuts were used, and found $R_{\text {loose }}=1.04 \pm 0.20$. When tight identification cuts were used, we observed a total of 18 events from the three processes and found $R_{\text {tight }}=0.96 \pm 0.23$. Their difference is $R_{\text {loose }}-R_{\text {tight }}=0.08 \pm 0.14$, where the errors are statistical only. Therefore, within errors, the results of the analysis do not depend on the detajls of the particle identification criteria.

Table 7.7. Breakdown of Monte Carlo events in categories with 4, 3 and 2 identified leptons for case ( $A$ ); I denotes an electron and/or muon.

\begin{tabular}{|c|c|c|c|c|c|}
\hline case (A) & $\begin{array}{r}\int L d t \\
\left(p b^{-1}\right)\end{array}$ & $\begin{array}{l}\text { \# Events } \\
\text { identified }\end{array}$ & $l l l l$ & $\| l l x$ & $l l x x$ \\
\hline$e^{+} e^{-} \rightarrow e^{+} e^{-} e^{+} e^{-}$ & 6005 & 360 & 125 & 208 & 27 \\
\hline$e^{+} e^{-} \rightarrow e^{+} e^{-} \mu^{+} \mu^{-}$ & 3682 & 194 & 56 & $\begin{array}{l}86 \text { еенx } \\
27 \mu \mu e x\end{array}$ & $25 e \mu x x$ \\
\hline$e^{+} e^{-} \rightarrow \mu^{+} \mu^{-} \mu^{+} \mu^{-}$ & 28343 & 118 & 25 & 67 & 26 \\
\hline$e^{+} e^{-} \rightarrow e^{+} e^{-} \tau^{+} \tau^{-}$ & 10055 & 14 & - & $\begin{array}{l}6 \text { eeex } \\
5 \text { eенx }\end{array}$ & $\begin{array}{c}3 \text { eex } x \\
-\end{array}$ \\
\hline
\end{tabular}

Various distributions are shown for the final samples, and compared with QED calculations for case (A). These distributions include all four tracks of an identified event, even when only three tracks, or two tracks of the simme charge sign a been 
Table 7.8. Brealdown of Monte Carlo events in categories with 4, 3 and 2 identified leptons for case (B); I denotes an electron and/or muon.

\begin{tabular}{|c|c|c|c|c|c|}
\hline case (B) & $\begin{array}{r}\int L d t \\
\left(p b^{-1}\right)\end{array}$ & $\begin{array}{l}\text { \# Events } \\
\text { identified }\end{array}$ & $m I I$ & $m l l x$ & $\| x x$ \\
\hline$e^{+} e^{-} \rightarrow e^{+} e^{-} e^{+} e^{-}$ & 6005 & 463 & 155 & 261 & 47 \\
\hline$e^{+} e^{-} \rightarrow e^{+} e^{-} \mu^{+} \mu^{-}$ & 3682 & 243 & 67 & $\begin{array}{l}106 е e \mu x \\
35 \mu \mu e x\end{array}$ & 35 enxx \\
\hline$e^{+} e^{-} \rightarrow \mu^{+} \mu^{-} \mu^{+} \mu^{-}$ & 28343 & 160 & 46 & 82 & 32 \\
\hline$e^{+} e^{-} \rightarrow e^{+} e^{-} \tau^{+} \tau^{-}$ & 10055 & 15 & - & $\begin{array}{l}5 \text { eeex } \\
4 \text { ee } \mu x\end{array}$ & 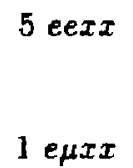 \\
\hline
\end{tabular}

individually identified. In such cases, the assumption of lepton number conservation determines the identity of the remaining tracks in the event. The energy and angular distribution of electrons $\left(e^{ \pm}\right)$in $e^{+} e^{-} e^{+} e^{-}$and $e^{+} e^{-} \mu^{+} \mu^{-}$events are shown in fig. 7.5 and fig. 7.6, whereas fig. 7.7 shows the momentum and angular distribution of muons $\left(\mu^{ \pm}\right)$in $e^{+} e^{-} \mu^{+} \mu^{-}$events. In fig. 7.8 we present the minimum mass distribution of two leptons. Here, we took the lowest invariant mass of all four possible combinations $\left(e^{+} e^{-}\right)$in $e^{+} e^{-} e^{+} e^{-}$events, and the lower invariant mass of the $e^{+} e^{-}$or $\mu^{+} \mu^{-}$combinations in $e^{+} e^{-} \mu^{+} \mu^{-}$events. The energy (total scalar momentum) distributions in (a) $e^{+} e^{-} e^{+} e^{-}$and (b) $e^{+} e^{-} \mu^{+} \mu^{-}$events are shown in fig. 7.9. The distributions of the invariant masses of the four $e^{+} e^{-}$pairs in $e^{+} e^{-} e^{+} e^{-}$events are shown in fig. 7.10 (a)-(d) in increasing mass order. Fig. 7.10 (a) shows the invariant mass distribution of the $e^{+} e^{-}$pair that has the smallest mass 
among the four pairs, whereas fig. 7.10 (d) shows the distribution of $e^{+} e^{-}$paits with the largest invariant mass in an event. The invariant mass distributions of the $e^{+} e^{-}$and the $\mu^{+} \mu^{-}$pairs in $e^{+} e^{-} \mu^{+} \mu^{-}$events are shown in fig. 7.11 (a) and (b), respectively. They all agree well with the QED predictions.
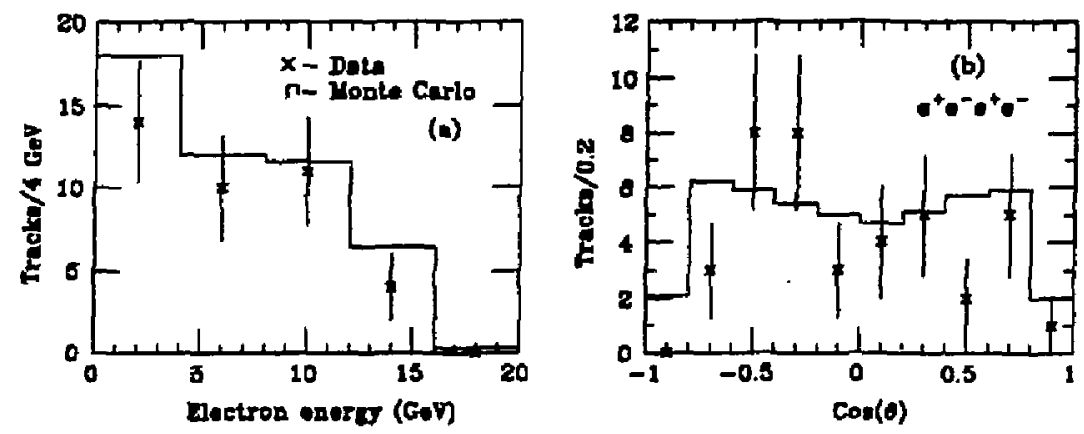

Figure 7.5. Distribution of electrons $\left(e^{ \pm}\right)$in $e^{+} e^{-} e^{+} e^{-}$events : (a) electron energy; (b) $\cos \theta$. The histogram is the QED prediction to $\alpha^{4}$ (Mark 11 data).

Finally, fig. 7.12 , fig. 7.13 and tig. 7.14 show 3 characteristic event pictures for each of the 3 processes $e^{+} e^{-} e^{+} e^{-}, e^{+} e^{-} \mu^{+} \mu^{-}$and $\mu^{+} \mu^{-} \mu^{+} \mu^{-}$. 

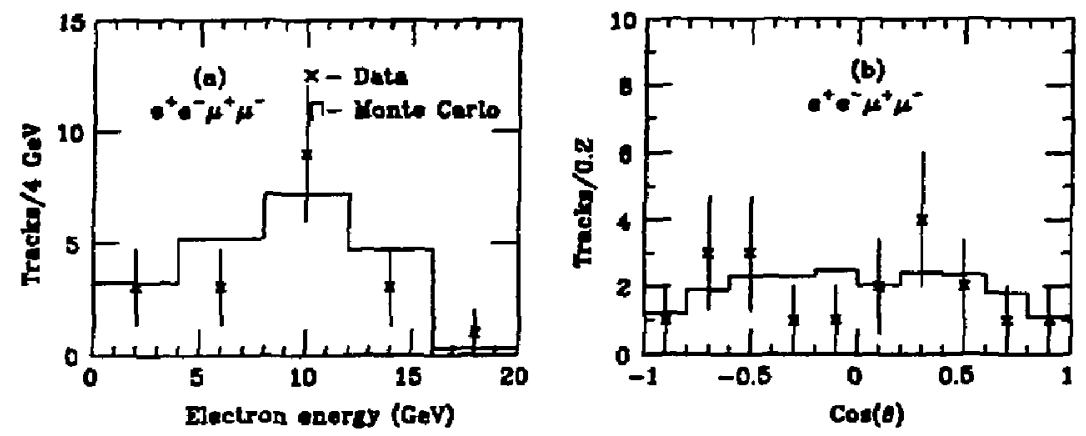

Figure 7.6. Distribution of electrons $\left(e^{ \pm}\right)$in $e^{+} e^{-} \mu^{+} \mu^{-}$events : (a) electron energy; (b) $\cos \theta$. The bistogram is the QED prediction to $\alpha^{4}$ (Mark II data).
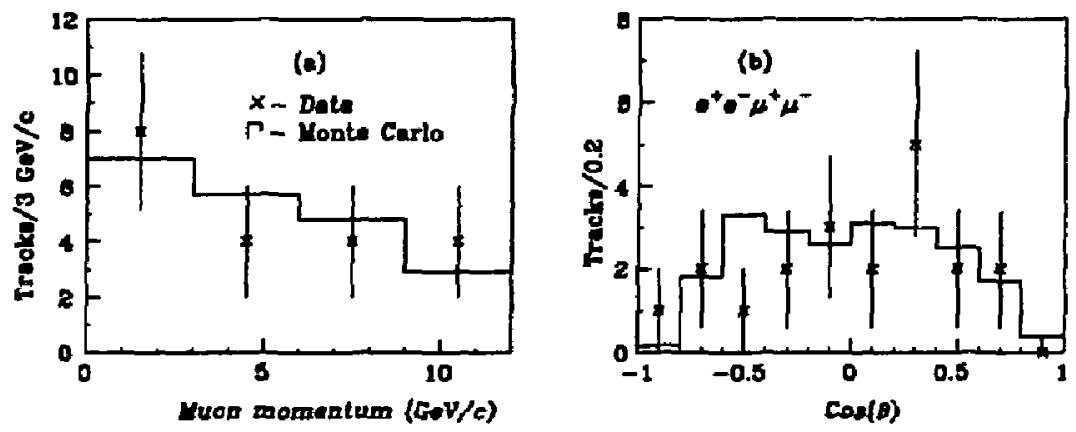

Figure 7.7. Ditribution of muon $\left(\mu^{ \pm}\right)$in $e^{+} e^{-} \mu^{+} \mu^{-}$events: (a) muon momentum; (b) $\cos (\theta)$. The histogram is the QED prediction to order $\alpha^{4}$ (Mark II data). 

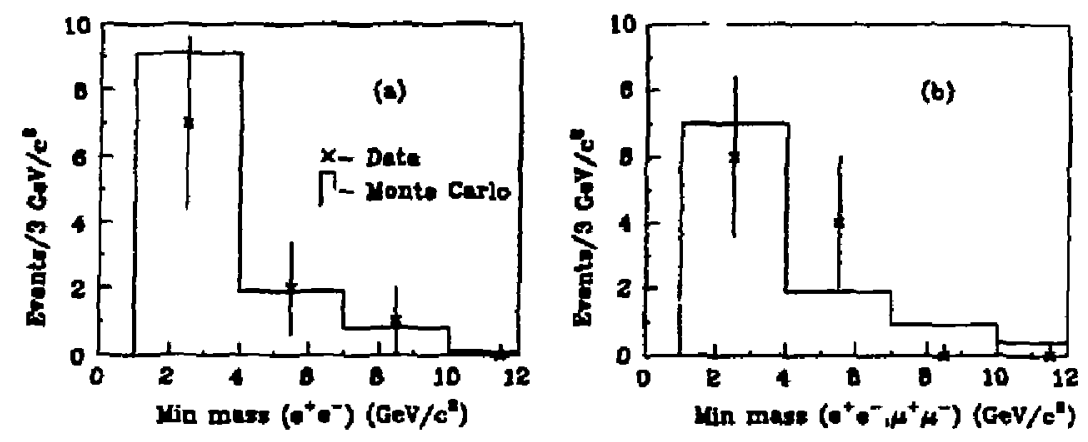

Figure 7.8. Lowest invariant mas distribution in (a) $e^{+} e^{-} e^{+} e^{-}$events; (b) $e^{+} e^{-} \mu^{+} \mu^{-}$ events. The histogram is the QED prediction to order $\alpha^{4}$ (Mark II date).
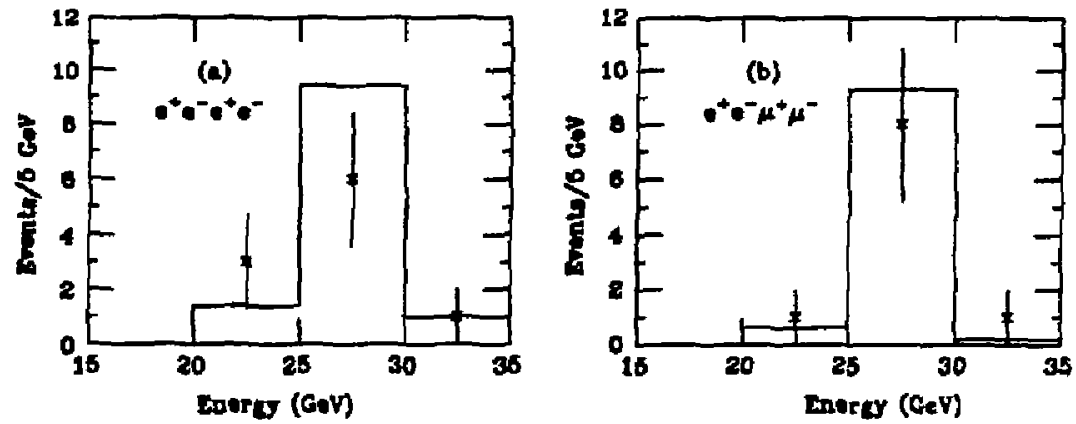

Figure 7.9. Energy distribution in (a) $e^{+} e^{-} e^{+} e^{-}$events; (b) $e^{+} e^{-} \mu^{+} \mu^{-}$events. The histogram is the QED prediction to order $a^{4}$ (Mark II data). 

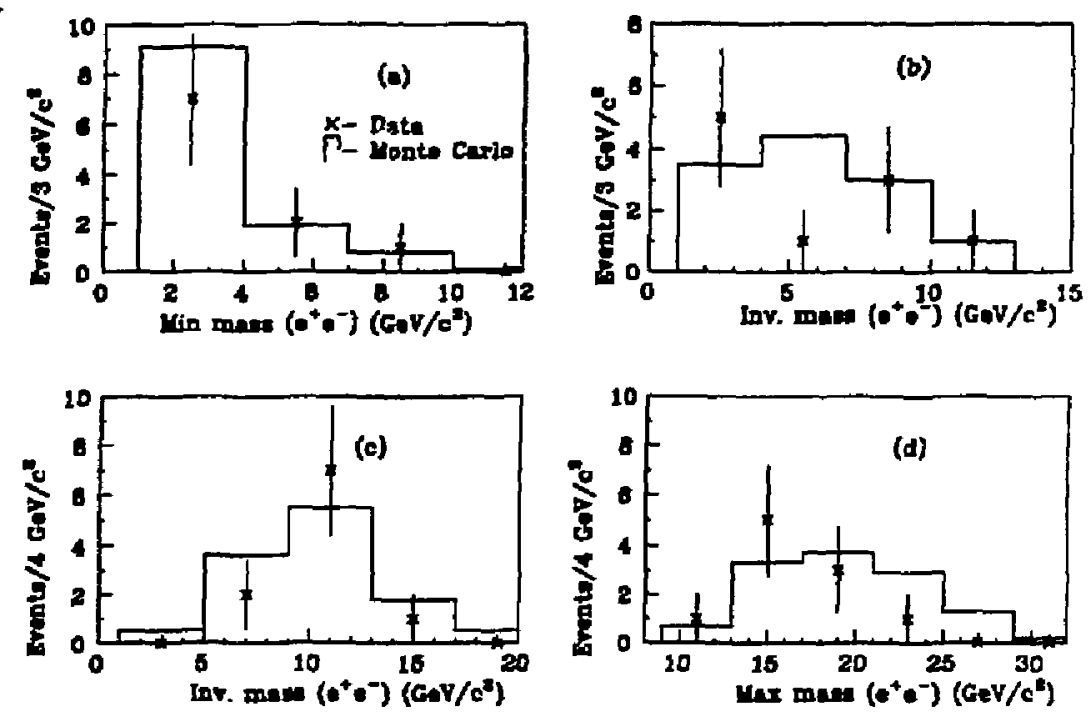

Figure 7.10. Invariant man distribution in $e^{+} e^{-} e^{+} e^{-}$events for all $e^{+} e^{-}$pairs in increasing tmas order (a)-(d) (Mark 11 data). 

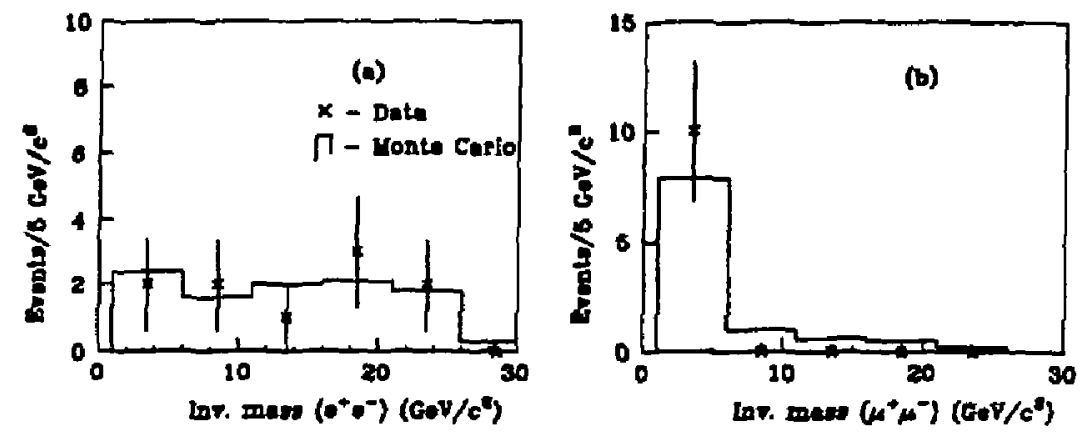

Figure 7.21. Invariant mas divtributions of the $e^{+} e^{-}$pair (a) and of the $\mu^{+} \mu^{-}$pair (b) in $e^{+} e^{-} \mu^{+} \mu^{-}$events (Mark 11 date).

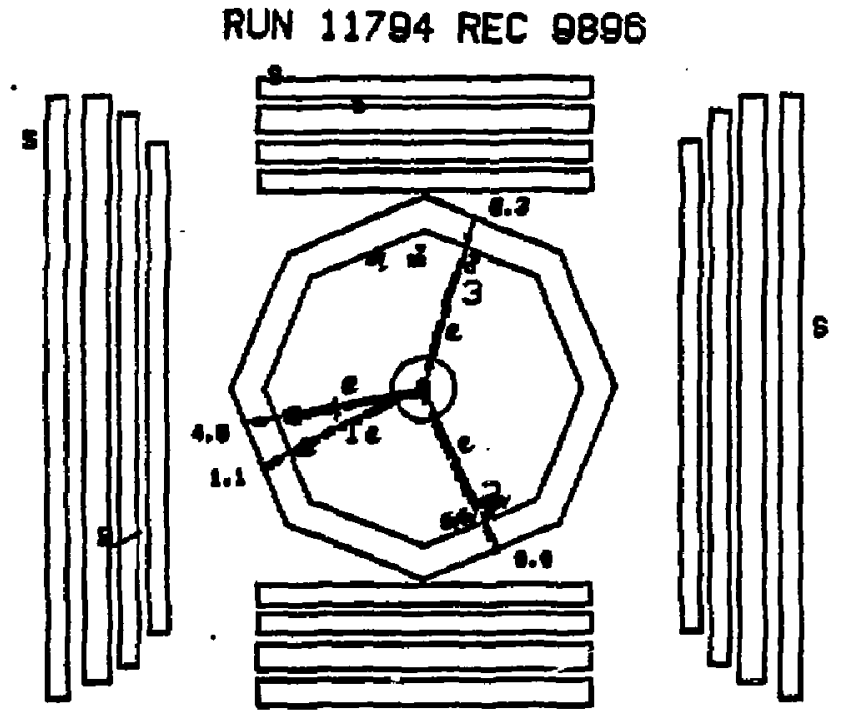

Figure 7.12. Characteriatic et $e^{-} e^{+} e^{-}$event pieture, observed in the Mark II data. 
RUN 12937 REC 3801
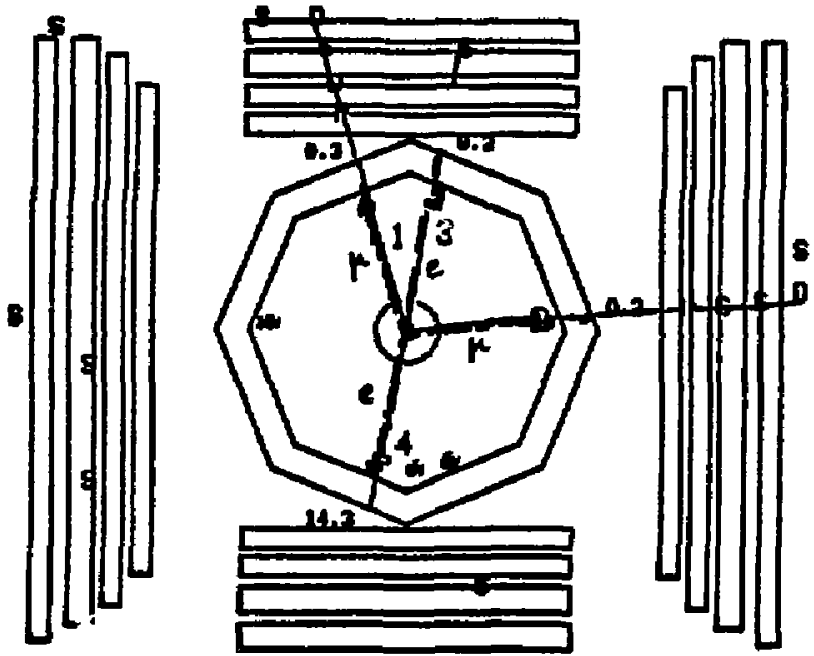

Figure 7.13. Characteriatic $e^{+} e^{-} \mu^{+} \mu^{-}$event picture, oberved in the Mark II data.

\section{RUN 10043 REC 3104}
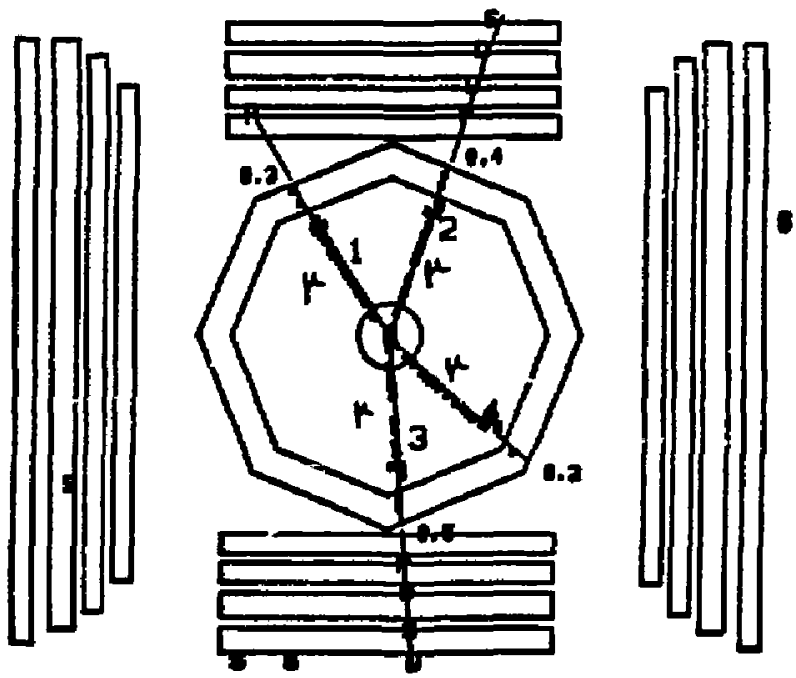

Figure 7.14. Picture of $\mu^{+} \mu^{-} \mu^{+} \mu^{-}$event obecrved in the Mark II date. 


\section{T.3 Detection efficiencies}

We applied kinematical cuts before detector simulation on the $M C$ generated four-momenta files, for each of the three processes $e^{+} e^{-} e^{+} e^{-}, e^{+} e^{-} \mu^{+} \mu^{-}$and $\mu^{+} \mu^{-} \mu^{+} \mu^{-}$. These cuts were identical to those used in section 7.1 for cases $(\dot{A})$ and (B). In detail, each event should satisfy the following criteria :

1. there should be 4 charged leptons with zero net total charge, (true by default)

2. each lepton should have a momentum $P \geq 0.5 \mathrm{GeV} / \mathrm{c}$ and $|\cos \theta| \leq 0.88$

3. at least two leptons should have $|\cos \theta| \leq \cos 45^{\circ}$

4. the sum of the scalar momenta of all four leptons should be $\geq 20 \mathrm{GeV} / \mathrm{c}$, but $\leq 40 \mathrm{GeV} / \mathrm{c}$

5. all opposite-charge pairs of leptons should have an invariant mass $\geq 1 \mathrm{GeV} / \mathrm{c}^{2}$

Table 7.9 lists the numbers of expected signal events, that were obtained by applying the above requirements, both before and after detector simulation, scaled down to the Mark II luminosity $L$ of $205 \mathrm{pb}^{-1}$. Note that $\mathrm{N}_{\text {after }}$ refers to the final results obtained after event identification, shown already in table 7.5 for $e^{+} e^{-} e^{+} e^{-}$, $e^{+} e^{-} \mu^{+} \mu^{-}$and $\mu^{+} \mu^{-} \mu^{+} \mu^{-}$. The errors attached are due to limited Monte Carlo statistics. The numbers corresponding to the MC generation luminosities, $600^{\circ}$ $p b^{-1}$ of $e^{+} e^{-} e^{+} e^{-}, 3682 p b^{-1}$ of $e^{+} e^{-} \mu^{+} \mu^{-}$, and $28343 p b^{-3}$ of $\mu^{+} \mu^{-} \mu^{+} \mu^{-}$, are given in parentheses. The detection efficiencies, determined from Monte Carlo, $\epsilon=\frac{N_{\text {after }}}{\text { Nbefore }_{\text {b }}}$, for $e^{+} e^{-} e^{+} e^{-}, e^{+} e^{-} \mu^{+} \mu^{-}$and $\mu^{+} \mu^{-} \mu^{+} \mu^{-}$, are $0.66 \pm 0.02$, $0.52 \pm 0.03$ and $0.54 \pm 0.03$, respectively.

In the same table, we present the cross sections $\sigma_{M C}$ (in $f b, 1 f b=10^{-s} \mathrm{pb}$ ) corresponding to the number of expected signal events for each of the 3 processes. The 
cross section $\sigma_{M C}$ is detived from the formula $\sigma_{M C}=\frac{N_{\text {before }}}{\mathrm{L}_{M C}}$, where $L_{M C}$ is the generation luminosity of the process, and $\mathrm{N}_{b e f o r e}$ is given in parentheses in table 7.9 .

Table 7.9. Summary of Mark II croes sections $\sigma_{M C}$ and Monte Carlo event statistics for case (A) (see text).

\begin{tabular}{|c|c|c|c|}
\hline case (A) & $\begin{array}{c}\sigma_{M C} \\
(\mathrm{fb})\end{array}$ & $\begin{array}{c}\mathrm{N}_{\text {before }} \\
(\mathrm{MC})\end{array}$ & $\begin{array}{c}\mathrm{N}_{\text {affer }} \\
(\mathrm{MC})\end{array}$ \\
\hline$e^{+} e^{-} e^{+} e^{-}$ & 91 & $18.6 \pm 0.8(545)$ & $10.9 \pm 0.7 \quad(360)$ \\
\hline$e^{+} e^{-} \mu^{+} \mu^{-}$ & 101 & $20.8 \pm 1.1(373)$ & $9.6 \pm 0.8 \quad(194)$ \\
\hline$\mu^{+} \mu^{-} \mu^{+} \mu^{-}$ & 8 & $1.6 \pm 0.1(219)$ & $0.76 \pm 0.07(118)$ \\
\hline
\end{tabular}

Table 7.10 has similar information, but in this case the requirement on the invariagt mass of opposite-charge pairs of leptons is $0.6 \mathrm{GeV} / \mathrm{c}^{2}$. An additional requirement demands that the mass of any three leptons be at least $1.6 \mathrm{GeV} / \mathrm{c}^{2}$. In this case, the detection efficiencies that are expected from Monte Carlo, for $e^{+} e^{-} e^{+} e^{-}, e^{+} e^{-} \mu^{+} \mu^{-}$and $\mu^{+} \mu^{-} \mu^{+} \mu^{-}$, are $0.62 \pm 0.02,0.48 \pm 0.02$ and $0.53 \pm 0.03$, respectively.

Table 7.10. Summary of Mark II croes sections $\sigma_{M C}$ and Monte Cario event statistics for cave (B) (see text).

\begin{tabular}{|c|c|c|c|}
\hline case (B) & $\begin{array}{c}\sigma_{M C} \\
(\mathrm{fb})\end{array}$ & $\begin{array}{c}N_{\text {before }} \\
(\mathrm{MC})\end{array}$ & $\begin{array}{c}\mathrm{N}_{\text {after }} \\
(\mathrm{MC})\end{array}$ \\
\hline$e^{+} e^{-} e^{+} e^{-}$ & 125 & $25.6 \pm 0.9(750)$ & $14.0 \pm 0.8 \quad(463)$ \\
\hline$e^{+} e^{-} \mu^{+} \mu^{-}$ & 137 & $28.2 \pm 1.2(306)$ & $12.0 \pm 0.9 \quad(243)$ \\
\hline$\mu^{+} \mu^{-} \mu^{+} \mu^{-}$ & 11 & $2.2 \pm 0.1 \quad(302)$ & $1.03 \pm 0.09(160)$ \\
\hline
\end{tabular}




\section{CHAPTER 8}

\section{HRS DATA ANALYSIS}

In this chapter, the experimental data from the HRS detector are compared with the QED calculations from chapters 4,5 and 6 . The first section presents the event selection of high $Q^{2}$ four-lepton candidate events. Comparisons between data and Monte Carlo expectations are presented next. Finally, we present a brief discussion of the detection efficiencies for all 3 signal processes, $e^{+} e^{-} e^{+} e^{-}, e^{+} e^{-} \mu^{+} \mu^{-}$, $\mu^{+} \mu^{-} \mu^{+} \mu^{-}$. The general scheme of this analysis is similar to the one previously presented for the Mark II detector.

\subsection{Selection criteria}

In the region of phase space that interests us (large angles, $\theta>25^{\circ}$, high $Q^{2}$ ), the presence of low backgrounds and the distinct signature of the four-lepton events allow us to use a combination of hard kinematical and rather loose identification criteria.

The analysis proceeded in two stages. First, an initial program filter ${ }^{(42)}$ selected events with 4 good charged tracks and zero net total charge.

From the original HRS sample of $(290.7 \pm 12.7) p b^{-x} 23203$ events survived. 
These events passed through a second and final analysis filter. This time, each event was required to satisfy the following criteria :

1. Four good charged tracks with zero net total charge. Each track was required to have a momentum of at least $0.6 \mathrm{GeV} / c,|\cos \theta| \leq 0.9$, and to pass close to the interaction point $(r \leq 0.05 \mathrm{~m}, z \leq 0.1 \mathrm{~m})$, through at least 6 drift chamber (DC) layers. All tracks, depending on their angle with respect to the beam axis, were required to have a corresponding minimum number of DC hits (NDC). Specifically, we asked that tracks satisfy the following conditions :

- for $0.866<|\cos \theta| \leq 0.9, N D C \geq 6$

- for $0.766<|\cos \theta| \leq 0.866, \mathrm{NDC} \geq 7$

- for $|\cos \theta| \leq 0.766, \mathrm{NDC} \geq 9$

2. The energy of an event, defined as the scalar sum of the momenta of the charged tracks times $c, \sum_{i=1,4} c\left|P_{i}\right|$, should be at least 20 , but not more than $40 \mathrm{GeV}$. This is an appropriate cut for reducing a large part of the background shown in fig. 6.1-6.5 (b). It also allows for radiation of photons from the initial or final states. The energy distribution of the data is shown in fig. 8.1 , in the form it had before this cut was applied.

3. All opposite-charge pairs must have an invariant mass of at least $1 \mathrm{GeV} / \mathrm{c}^{2}$. This cut not only ensures that we are testing QED at small distances, but it also belps reduce background coming from hadronic resonance production and single-photon conversion. Figure 8.2 shows the invariant mass distribution of the data, in the form it had before this cut was applied. The invariant masses 
shown range from 0 to $1.5 \mathrm{GeV} / \mathrm{c}^{2}$.

4. In each event, there should be at least 3 identified leptons, or 2 identified leptons of the same charge sign. The identity of the remaining tracks in an event is determined by assuming lepton flavor conservation. In this case, we explicitly demand that the identification assignment of the tracks is consistent with that made from the assumption of lepton flavor conservation. For example, if there are two tracks identifie $e^{t} ;$ electrons and one as a muon, the event is a candidate $e^{+} e^{-} \mu^{+} \mu^{-}$as long as the fourth track's identification assignment does not contradict the muon identity. In order to be identified, lepton-candidates must have a momentum of at least $1 \mathrm{GeV} / \mathrm{c}$. The electron and muon candidates were selected on the basis of information obtained from the drift chambers and the shower counters.

The effect of each cut on the original data sample is given in table 8.1

Table 8.1. Effect of the analysis cuts on HRS data.

\begin{tabular}{|l|c|c|c|c|}
\hline & Cut 1 & Cut 2 & Cut 3 & Cut 4 \\
\hline \# of Events & 7224 & 2557 & 73 & 43 \\
\hline
\end{tabular}

Tracks were identified by examining the ratio $\mathrm{E} / \mathrm{P}$ of the energy $\mathrm{E}$ deposited in the shower counters over the momentum $P$ measured by the drift chamber. This energy $E$ of a particle should not be confused with the energy of an event, defined earlier in this section. Figure 8.3 shows the E/P distribution of tracks for all events which survived the kinematical cuts 1-3. 
For an electron, which may lose all its energy when passing through material of several radiation lengths thick, this ratio should be about one. For a minimum ionizing particle with momentum above $1 \mathrm{GeV} / \mathrm{c}$, this ratio should be at most 0.2 , taking into account that a non-showering particle typically deposits an energy of $0.2 \mathrm{GeV}$ in the shower counters. ${ }^{(24)}$

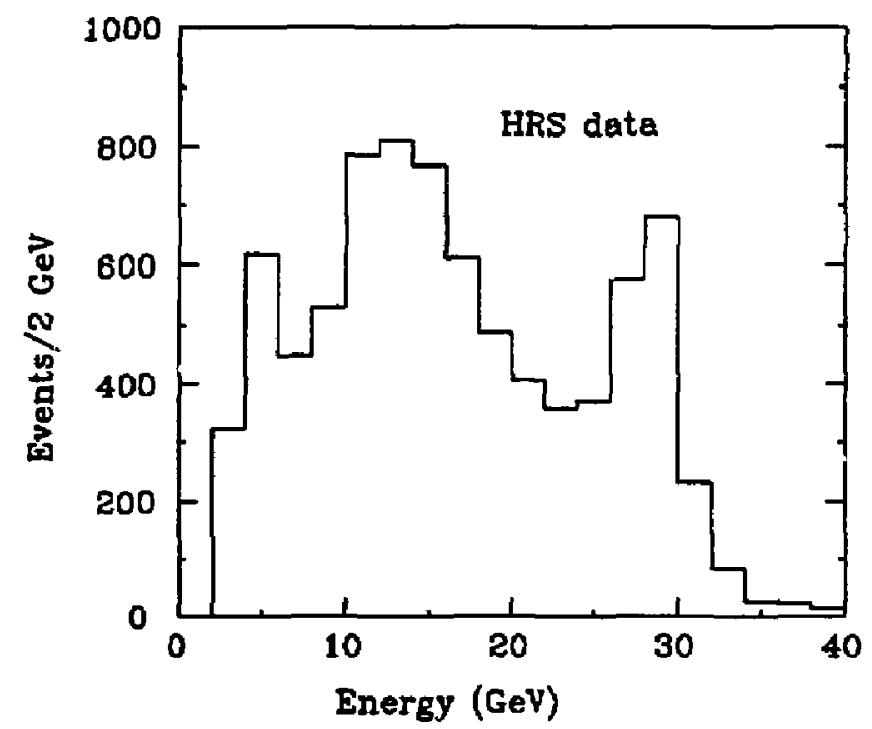

Figure 8.1. Energy distribution before cut (2) is applied on HRS data.

Tracks extrapolated into the end cap or barrel shower counters were assigned the energy (if any) deposited in the module they bit. A candidate lepton was required to have $0.55<\mathrm{E} / \mathrm{P}<1.45$ in order to be identified as an electron. So, for electrons with a momentum of at least $1 \mathrm{GeV} / \mathrm{c}$, we ask that their $\mathrm{E} / \mathrm{P}$ be $\pm 3 \sigma$ away from $\mathrm{E} / \mathrm{P}=1$. A track with $\mathrm{E} / \mathrm{P} \leq 0.55$ was identified as a muon, as long as it depoṣited less than $0.5 \mathrm{GeV}$ in the acceptance region of either shower counter. The latter condition was imposed in order to ensure a reliable measurement of the deposited 


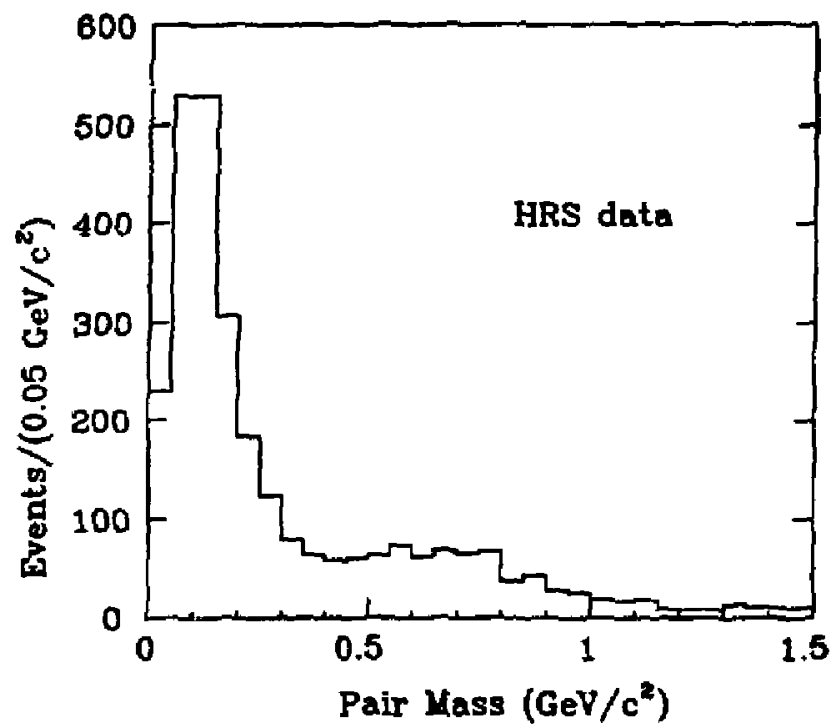

Figure 8.2. Distribution of the invariant mass of all opposite-charge pairs in HRS data, before cut (3) is applied.

energy. The barrel shower counter covers the angular region $|\cos \theta| \leq 0.6$. A track hitting a barrel module was required to be at least $0.5^{\circ}$ from the edge at the radia] distance of $2.03 \mathrm{~m}$. In addition we required that the distance in 2 between the track that was extrapolated into a barrel module and the center-of-gravity of the energy deposited in the module be not more than $0.5 \mathrm{~m}$. The end cap shower counter covers the angular region of $0.7 \leq|\cos \theta| \leq 0.9$. A track hitting an end cap module was required to be at least $0.5 \mathrm{~cm}$ away from the edge.

The energy distribution of tracks from all events surviving cut 3 is shown in fig. 8.4, while the energy deposited in the shower counters by tracks with $\mathrm{E} / \mathrm{P} \leq 0.55$ is presented in the range $0-2 \mathrm{GeV}$, in fig. 8.5 .

The list of the 73 events which passed all kinematical requirements, some of their properties, and their identification assignments are given in appendix $D$. 
88

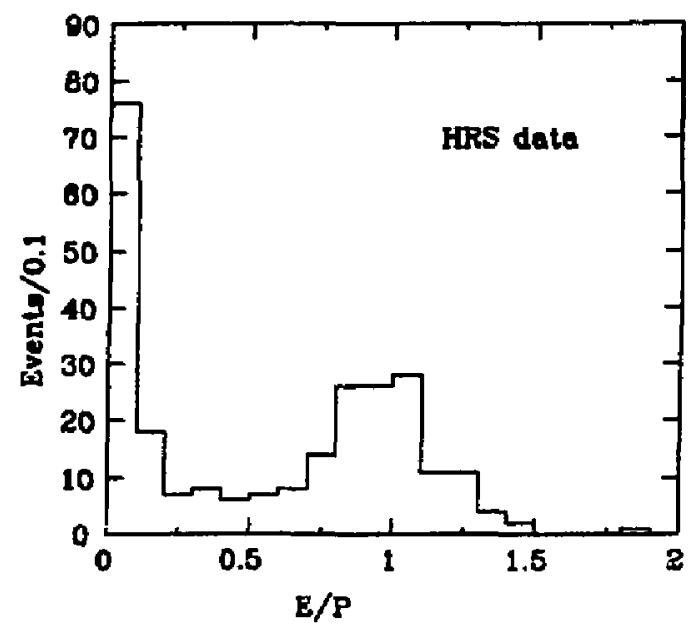

Figure 8.3. E/P dietribution of tracks from ell events observed in the BRS dale ster cut (3).

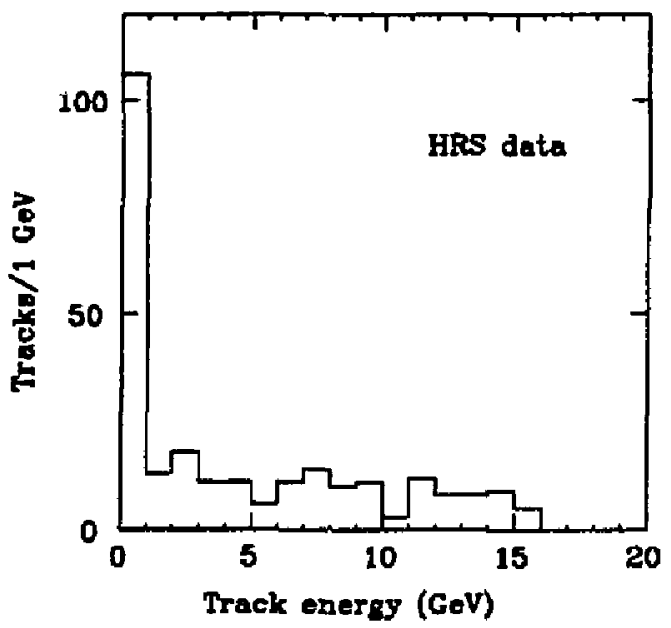

Figure 8.4. Energy diatribution of individual tracks in HRS four-lepton candidates.

Of the 73 events, 43 events were identified by our identification algorithrr. Seventeen were ideatified as $e^{+} e^{-} e^{+} e^{-}$, twenty-four as $e^{+} e^{-} \mu^{+} \mu^{-}$and two as $\mu^{+} \mu^{-} \mu^{+} \mu^{-}$. Of the $17 e^{+} e^{-} e^{+} e^{-}$events, 3 had all four leptons identified as electrons, 11 had three, and 3 events had two leptons of the same charge sign identified as electruns. Of the 


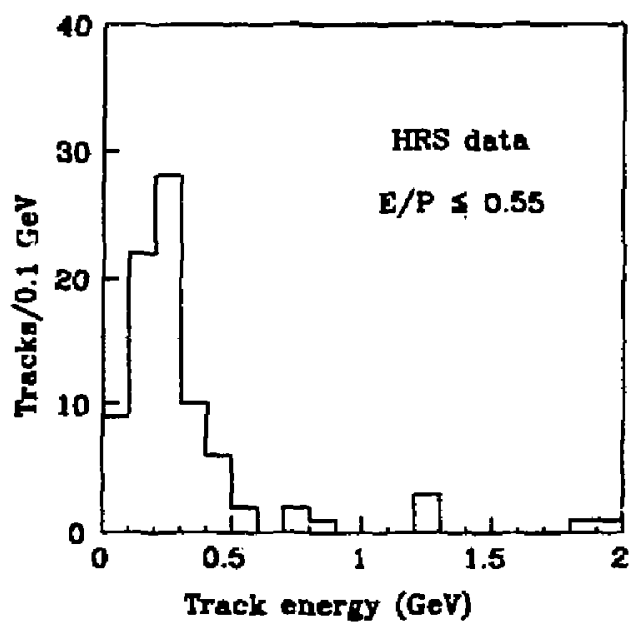

Figure 8.5. Energy distribution of individual tracks witb $E / P \leq 0.55$ in RRS four-lepton candidster.

$24 e^{+} e^{-} \mu^{+} \mu^{-}$events, 3 had all four leptons identified, 20 had three, and 1 event had two leptons of the same charge sign identified. Of the two $\mu^{+} \mu^{-} \mu^{+} \mu^{-}$candidates, one was rejected upon further examination. It was Run 3953, Record 8637, which had one track with a mismeasured momentum of $27.7 \mathrm{GeV} / \mathrm{c}$. All four tracks hit two neighboring modules in the west end cap shower counter. The event also contained a $17.3 \mathrm{GeV}$ measured energy deposit in the east end cap not correlated with any of the charged tracks. This topology is typical of annihilation processes into hadrons which are preceded by initial-state radiation of a large-angle photon. The other $\mu^{+} \mu^{-} \mu^{+} \mu^{-}$event had all four leptons identified as muons.

\subsection{Comparison with Monte Carlo}

As mentioned in earlier chapters, the Monte Carlo programg ${ }^{(4-7)}$ of Berends et al. were used to generate $e^{+} e^{-} e^{+} e^{-}, e^{+} e^{-} \mu^{+} \mu^{-}$and $\mu^{+} \mu^{-} \mu^{+} \mu^{-}$events. Not only did the kinematic cuts used for the generation of the four-lepton final states 
extend a few standard deviations beyond the final acceptance criteria (see chapters 4, 5 and 6), but also the luminosities of the Monte Carlo event samples sufficiently exceeded the actual HRS luminosity of $291 p^{-1}$. We present the effect of the analysis cuts on Monte Carlo generated signal and background events in table 8.2. For each process, the number of events surviving each cut are listed under the corresponding column, while the initial number of events is given in the second column. One other source of background, $e^{+} e^{-} \gamma \gamma$, is estimated to be negligible, not only on the basis of the Mark II analysis, but also because of the application of a $1 \mathrm{GeV} / \mathrm{c}^{2}$ invariant mass cut on any opposite-charge pair of tracks.

Table 8.2. Effect of BRS analysis cuts on MC signal and background events. The integrated luminceity corregponding to each process in given in $p^{-1}$.

\begin{tabular}{|c|c|c|c|c|c|c|}
\hline & $\int L d t$ & \# Events & Cut 1 & Cut 2 & Cut 3 & ID \\
\hline$e^{+} e^{-} \rightarrow e^{+} e^{-} e^{+} e^{-}$ & 1598 & 700 & 308 & 307 & 189 & 132 \\
\hline$e^{+} e^{-} \rightarrow e^{+} e^{-} \mu^{+} \mu^{-}$ & 1590 & 800 & 363 & 362 & 225 & 176 \\
\hline$e^{+} e^{-} \rightarrow \mu^{+} \mu^{-} \mu^{+} \mu^{-}$ & 2059 & 50 & 31 & 31 & 22 & 21 \\
\hline$e^{+} e^{-} \rightarrow e^{+} e^{-} \tau^{+} \tau^{-}$ & 2530 & 306 & 5 & 0 & 0 & 0 \\
\hline$e^{+} e^{-} \rightarrow e^{+} e^{-} h^{+} h^{-}$ & 933 & 597 & 3 & 1 & 1 & 0 \\
\hline$e^{+} e^{-} \rightarrow g \bar{q} \rightarrow$ hadrons & 185 & 74005 & 360 & 41 & 0 & 0 \\
\hline$e^{+} e^{-} \rightarrow \tau^{+} \tau^{-}$ & 294 & 39984 & 4339 & 514 & 2 & 1 \\
\hline
\end{tabular}

The number of predicted events from Monte Carlo was $23.5 \pm 2.2,31.5 \pm 2.5$ and $2.9 \pm 0.7$ for $e^{+} e^{-} e^{+} e^{-}, e^{+} e^{-} \mu^{+} \mu^{-}$and $\mu^{+} \mu^{-} \mu^{+} \mu^{-}$, respectively, while 17, 24 and 1 events were observed. The errors attached to the predicted values are statistical only. 
A summary of the HRS results for the four-lepton signal and background reactions is shown in table 8.3. The number of data events are presented without background subtraction. The numbers of expected sigaal events have been corrected for initial-state radiation (see appendix A). The errors in the total Monte Carlo events are statistical (first error) and systematic (second error). The systematic error comes from the uncertainty in the luminosity measurement $(4 \%)$.

Among the observed identified events we searched for photons with an energy greater than $1 \mathrm{GeV}$ and an angle greater than $10^{\circ}$ with the nearest track. There was one event which radiated such a photon. It was an $e^{+} e^{-} \mu^{+} \mu^{-}$candidate event with a photon of $4.6 \mathrm{GeV}$ of energy that made an angle of $31^{\circ}$ with the nearest irack. This event cannot be compared separately with theory, since there is no complete QED calculation ${ }^{(39)}$ for the radiative corrections to order $a^{4}$. It is shown in fig. 8.6.
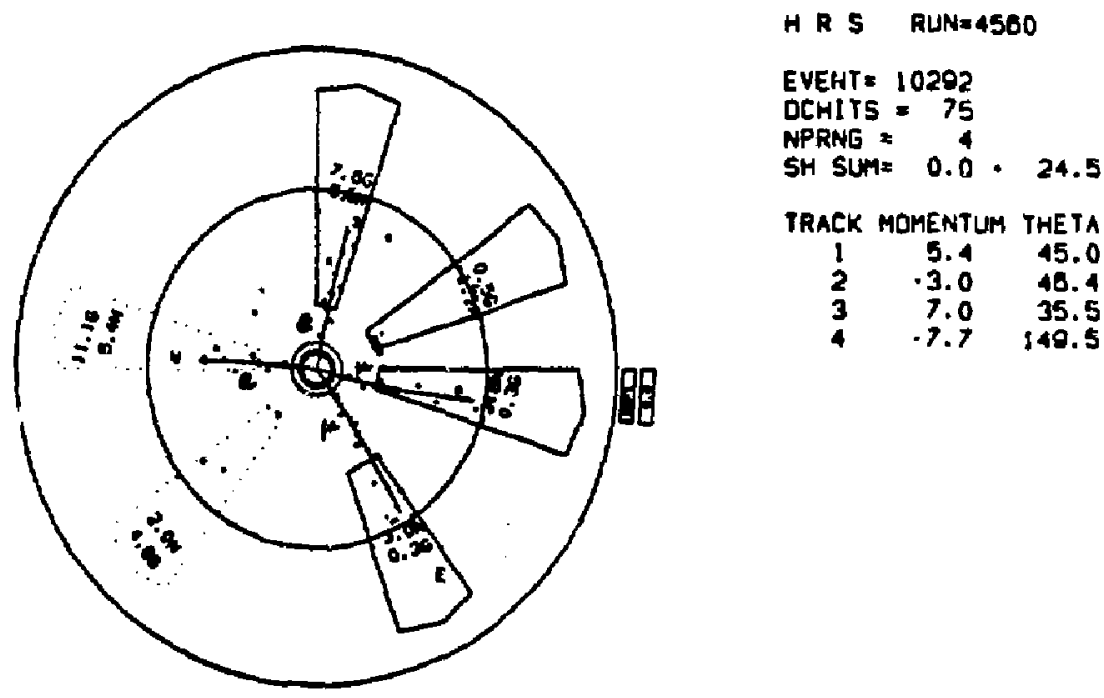

Figute 8.6. BRS $\alpha^{8} e^{+} e^{-} \mu^{+} \mu^{-}$candidale event. 
Table B.3. Number cf observed (no background uubtraction) and expected aignel and background eveats for HRS $e^{+} e^{-} e^{+} e^{-}, e^{+} e^{-} \mu^{+} \mu^{-}$and $\mu^{+} \mu^{-} \mu^{+} \mu^{-}$(pair mames $\geq 1 \mathrm{GeV} / \mathrm{c}^{2}$ ). Shown upper limits are at 90\% CL.

\begin{tabular}{|c|c|c|c|c|}
\hline & $e^{+} e^{-} e^{+} e^{-}$ & $e^{+} e^{-} \mu^{+} \mu^{-}$ & $\mu^{+} \mu^{-} \mu^{+} \mu^{-}$ & $\begin{array}{c}\text { events failing } \\
\text { lepton } \\
\text { ID cut }\end{array}$ \\
\hline Data events & 17 & 24 & 1 & 31 \\
\hline Expected signal & & & & \\
$e^{+} e^{-} \rightarrow e^{+} e^{-} e^{+} e^{-}$ & 23.5 & & & 10.4 \\
$e^{+} e^{-} \rightarrow e^{+} e^{-} \mu^{+} \mu^{-}$ & \pm 2.2 & & & \pm 1.4 \\
$e^{+} e^{-} \rightarrow \mu^{+} \mu^{-} \mu^{+} \mu^{-}$ & & 31.5 & & 8.9 \\
& & \pm 2.5 & & \pm 1.3 \\
\hline Expected background & & & 2.9 & 0.14 \\
$e^{+} e^{-} \rightarrow e^{+} e^{-} \tau^{+} \tau^{-}$ & $<0.2$ & $<0.2$ & $<0.2$ & $<0.2$ \\
$e^{+} e^{-} \rightarrow e^{+} e^{-} h^{+} h^{-}$ & $<0.7$ & $<0.7$ & $<0.7$ & 0.3 \\
& & & & \pm 0.3 \\
$e^{+} e^{-} \rightarrow \tau^{+} \tau^{-}$ & $<2.2$ & 1.0 & $<2.2$ & 1.0 \\
$e^{+} e^{-} \rightarrow q \bar{q}$ & $<3.6$ & $<3.6$ & $<3.6$ & $<3.6$ \\
\hline Total & 23.5 & 32.5 & 2.9 & 20.7 \\
\hline expected events & \pm 2.2 & \pm 2.7 & \pm 0.7 & \pm 2.2 \\
& \pm 0.04 & \pm 0.04 & \pm 0.04 & \pm 0.04 \\
\hline
\end{tabular}


The total number of Monte Carlo signal and background events, taking into account those events which failed the lepton identification criteria, is $\mathrm{N}_{M C}^{\cdot}=79.6=23.5+32.5+2.9+20.7$ (from table 8.3). The corresponding total number of data events is $N_{D}=73=17+24+1+31$. Since the expected contribution from background processes is small (about $3 \%$ ), a direct comparison of $\mathrm{N}_{M C}$ and $\mathrm{N}_{D}$ represents a sensitive test of QED. We see that there is agreement within one standard deviation between data and Monte Carlo results, before lepton identification.

After lepton identification, the agreement between the data and the Monte Carlo results is not quite as good, the data falling below expectation in all channels. Even though this is not highly significant, it was studied further by comparing the data and Monte Carle distributions for E/P and deposited energy. These distributions were used for the identification of electrons and muors. Figure 8.7 (b) shows the $\mathrm{E} / \mathrm{P}$ distribution of tracks from Monte Carlo $e^{+} e^{-} e^{+} e^{-}, e^{+} e^{-} \mu^{+} \mu^{-}$and $\mu^{+} \mu^{-} \mu^{+} \mu^{-}$events, scaled down to $291 \mathrm{pb}^{-1}$, before lepton identification. This is to be compared with the E/P distribution of data tracks shown in fig. 8.7 (a), which is identical to fig. 8.3 , and is presented here once again for the sake of comparison. Figure 8.8 (b) shows the energy distribution of tracks with $E / P \leq 0.55$ from Morte Carlo $e^{+} e^{-} e^{+} e^{-}, e^{+} e^{-} \mu^{+} \mu^{-}$and $\mu^{+} \mu^{-} \mu^{+} \mu^{-}$events, scaled down to $291 p^{-1}$, before lepton identification. For the sake of comparison again, we present fig. 8.8 (a), the corresponding distribution of data tracks, which is identical to fig. 8.5, shown earlier. These distributions are qualitatively similar but both Monte Carlo distribu- 
tions show slightly narrower peaks than those present in the data. The somewhat weaker agreement between the number of data and Monte Carlo events after lepton identification, may be caused, in part, by small imperfections in the simulation of the HRS calorimeter response to electrons and muons. However, we reiterate that the general agreement is good and that the relatively small data sample does not allow a deeper investigation of this point.
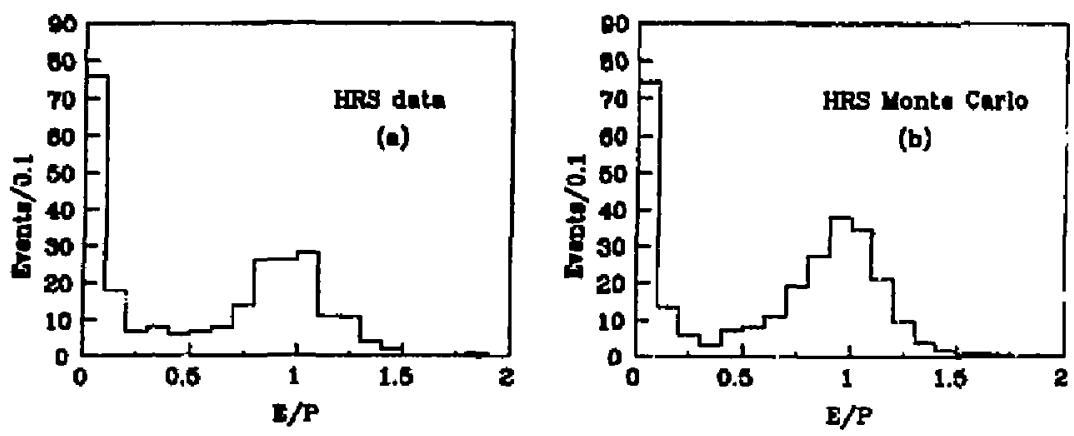

Figure 8.7. E/P distribution of tracks before lepton identification in HRS data four-lepton candidates (a) and in Monte Carlo four-lepton eventa (b).
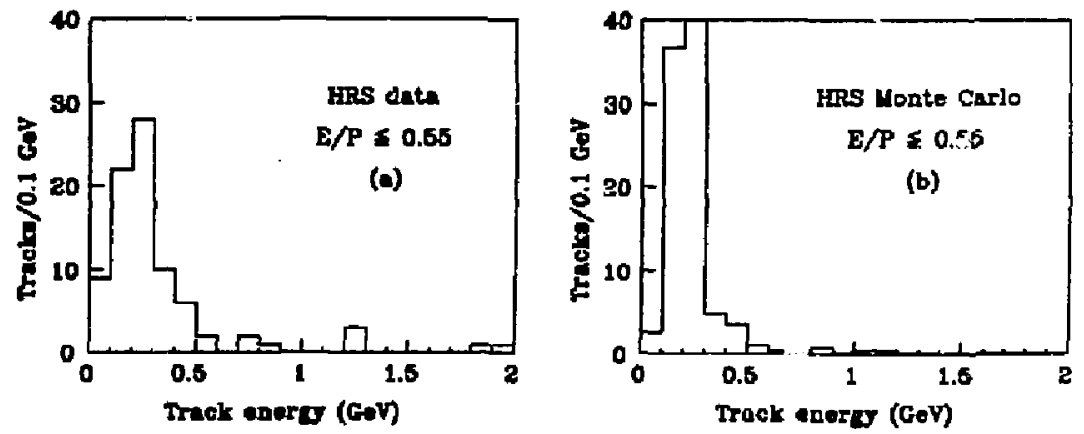

Figure 8.8. Energy diatribution of individual tracks with $E / P \leq 0.55$ in $B$ RS data four-lepton candidates (a) and in Monte Carlo four-lepton events (b). 
Various distributions are shown for the final HRS samples, and compared with QED calculations. All four tracks of an identified event are included in these distributions, even when only three or two of the same charge sign tracks have been identified. In such events, the identity of the remaining tracks is determined by the assumption of lepton number conservation. The energy and angular distributions of electrons ( $\left.e^{ \pm}\right)$in $e^{+} e^{-} e^{+} e^{-m}$ and $e^{+} e^{-} \mu^{+} \mu^{-}$events are shown in fig. 8.9 and fig. 8.10 , respectively. The momentum and angular distribution of muons $\left(\mu^{ \pm}\right)$in $e^{+} e^{--} \mu^{+} \mu^{-}$events is shown in fig. 8.11. In fig. 8.12 we present the minimum mass distribution of two leptons. We took the lowest invariant mass of all four possible combinations $\left(e^{+} e^{-}\right)$in $e^{+} e^{-} e^{+} e^{-}$events, and the lower invariant mass of the $e^{+} e^{-}$ or $\mu^{+} \mu^{-}$combinations in $e^{+} e^{-} \mu^{+} \mu^{-}$events. The energy (total scalar momentum) distributions in (a) $e^{+} e^{-} e^{+} e^{-}$and (b) $e^{+} e^{-} \mu^{+} \mu^{-}$events are shown in fig. 8.13. The distributions of the invariant masses of the four $e^{+} e^{-}$pairs in $e^{+} e^{-} e^{+} e^{-}$events are shown in fig. 8.14 (a)-(d) in increasing mass order. Fig. 8.14 (a) shows the invariant mass distribution of the $e^{+} e^{-}$pair with the smallest mass among the four pairs, whereas fig. 8.14 (d) shows the digtribution of $e^{+} e^{-}$pairs with the largest invariant mass in an event. The invariant mass distributions of the $e^{+} e^{-}$and the $\mu^{+} \mu^{-}$ pairs in $e^{+} e^{-} \mu^{+} \mu^{-}$events are shown in fig. 8.15 (a) and (b), respectively. They all agree reasonably well with the QED predictions. Finally, fig. 8.16, fig. 8.17 and fig. 8.18 show 3 characteristic event pictures for each of the 3 processes $e^{+} e^{-} e^{+} e^{-}$, $e^{+} e^{-} \mu^{+} \mu^{-}$and $\mu^{+} \mu^{-} \mu^{+} \mu^{-}$. 

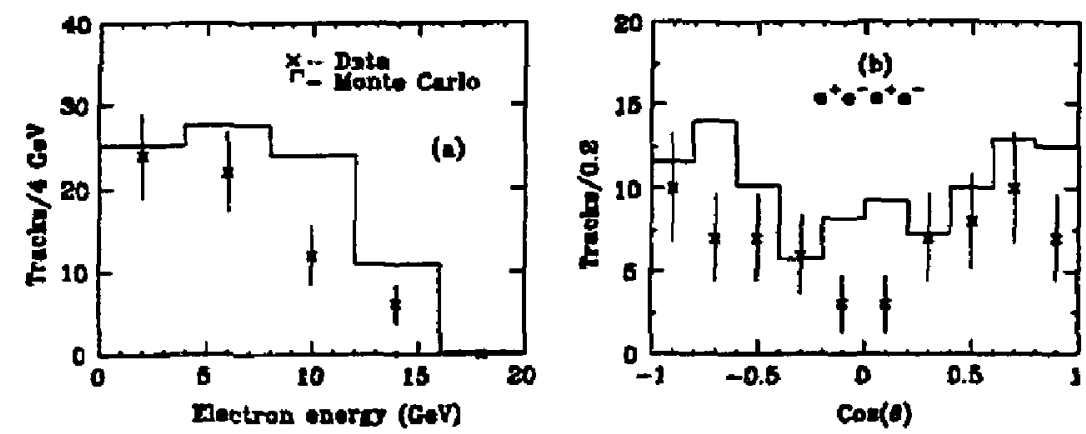

Figure B.9. Distribution of ejectrons $\left(e^{ \pm}\right)$in $e^{+} e^{-} e^{+} e^{-}$events : (b) electron energy;(b) $\cos \theta$. The bistogram is the QED prediction to $\alpha^{4}$ (HRS data).
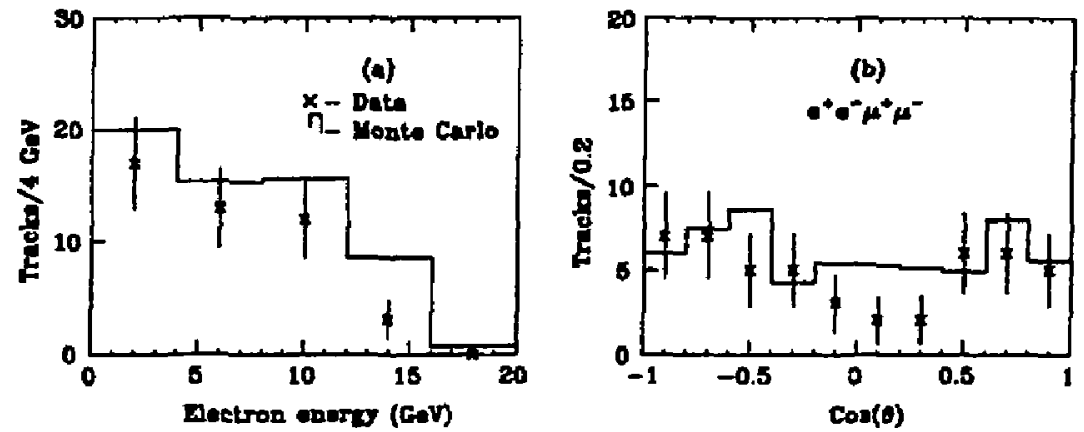

Figure 8.10. Distribution of electronn $\left(e^{ \pm}\right)$in $e^{+} e^{-} \mu^{+} \mu^{-}$events : (a) electron energy; (b) $\cos \theta$. The histogram is the QED prediction to $\alpha^{4}$ (HRS data). 

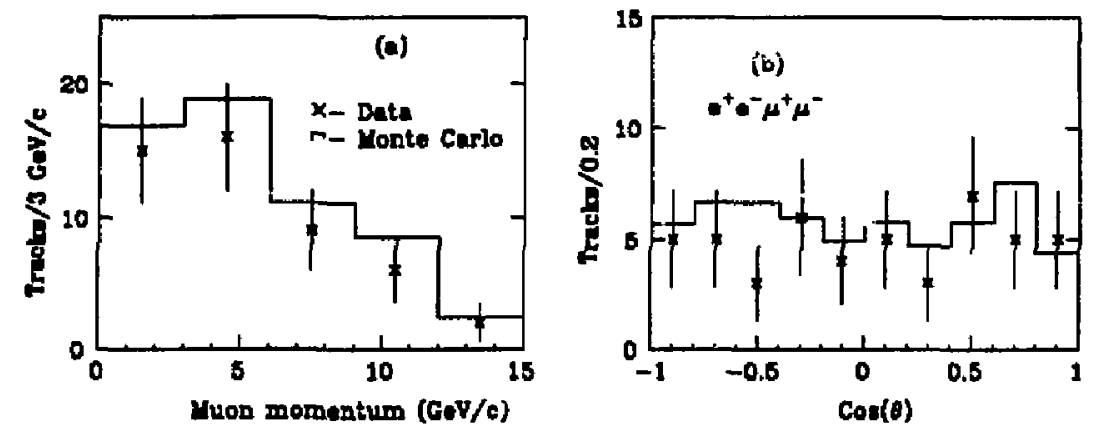

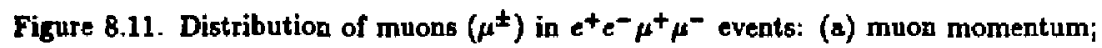
(b) $\cos (\theta)$. The histogram in the QED prediction to order $\alpha^{4}$ (HRS data).
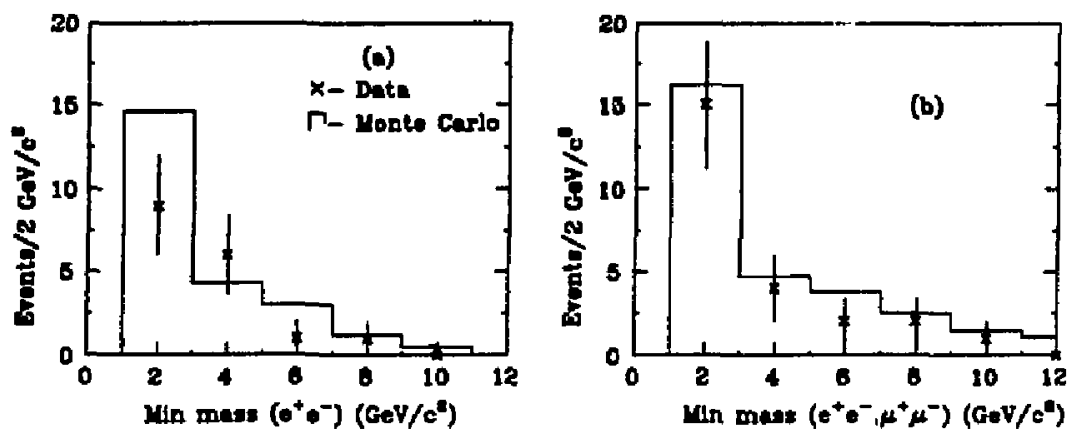

Figure 8.12. Loweat invariant mas diatribution in (a) $e^{+} e^{--} e^{+} e^{-}$events; (b) $e^{+} e^{-} \mu^{+} \mu^{-}$ events. The hintogram is the QED prediction to order $\alpha^{-1}$ (BRS data). 

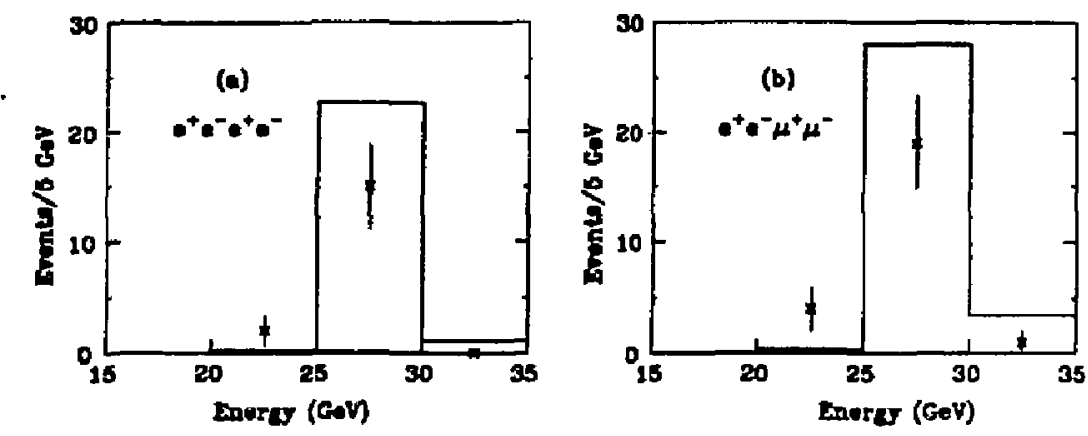

Figure 8.13. Energy distribution in (a) $e^{+} e^{-} e^{+} e^{-}$events; (b) $e^{+} e^{-} \mu^{+} \mu^{-}$events. The histogram is the QED prediction to order $\alpha^{4}$ (HRS data).
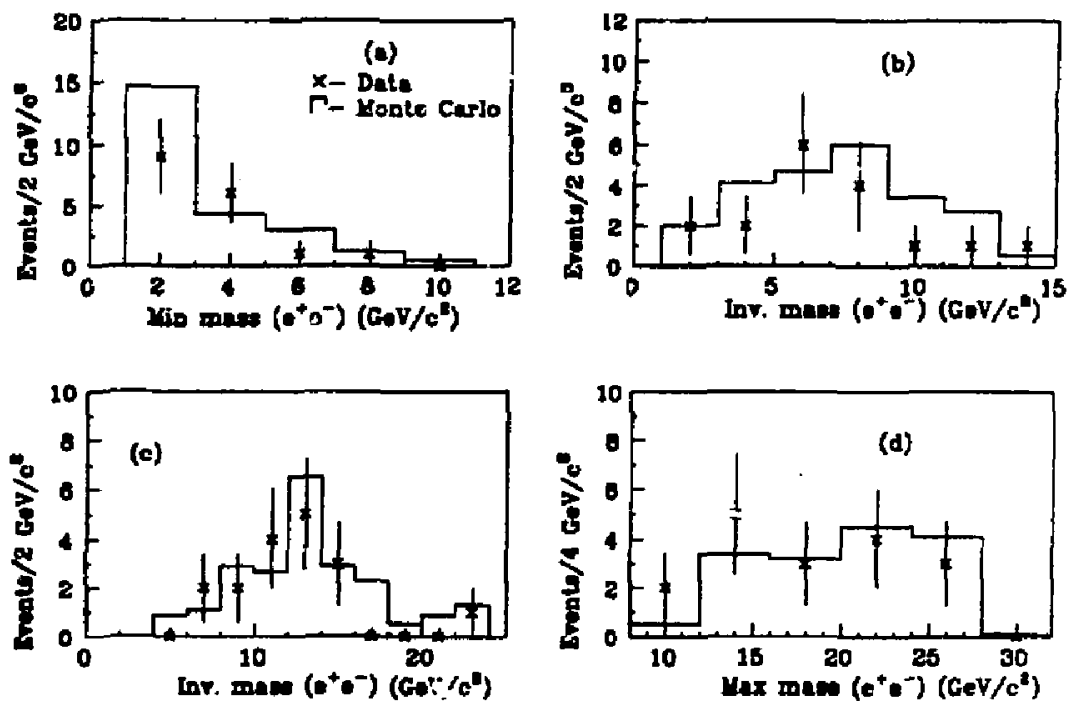

Figure 8.14. Invariant mas distribution in $e^{+} e^{-} e^{+} e^{-}$events for all $e^{+} e^{-}$pairs in in. creasing mass order (a)-(d) (BRS data). 

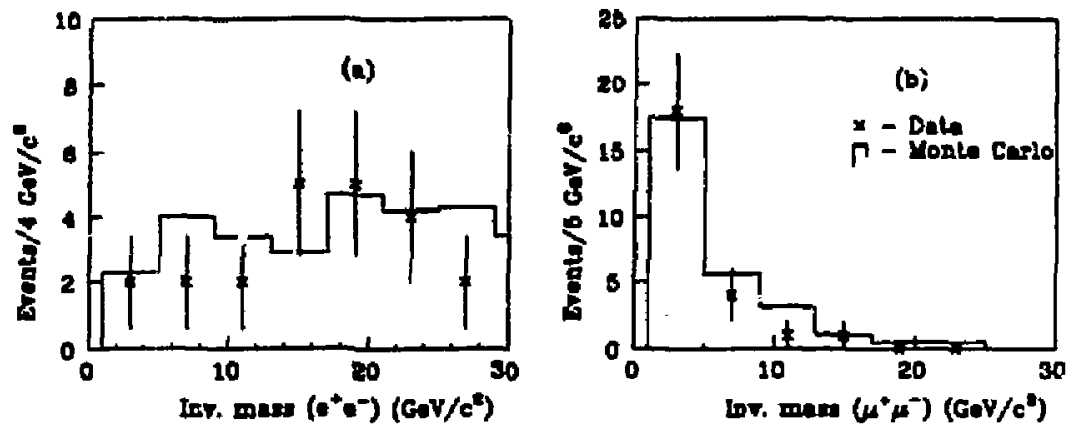

Figure 8.15. Invariant man dintributions of the $e^{+} e^{-}$pair (a) and of the $\mu^{+} \mu^{-}$pair (b) in $e^{+} e^{-} \mu^{+} \mu^{-}$events (BRS date).

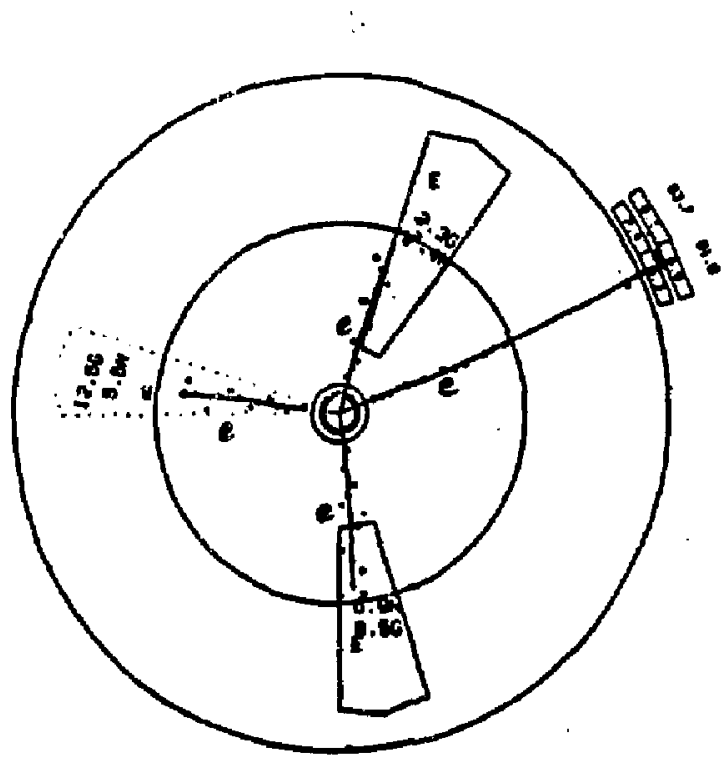

H R $S$ RUM- 1173

EVENT. 2800

DCHITS = 60

NPRNG.

SH SUM. $0.2 \cdot 23.5$

TRACK MOMENTUM THETA

$\begin{array}{rrr}1 & .5 .7 & 81.4 \\ 2 & 0.2 & 40.0 \\ 3 & 3.0 & 43.4 \\ 4 & -12.2 & 143.1\end{array}$

Figure 8.10. Charecterintic $e^{+} e^{-} e^{+} e^{-}$event picture abserved in the HRS data. 


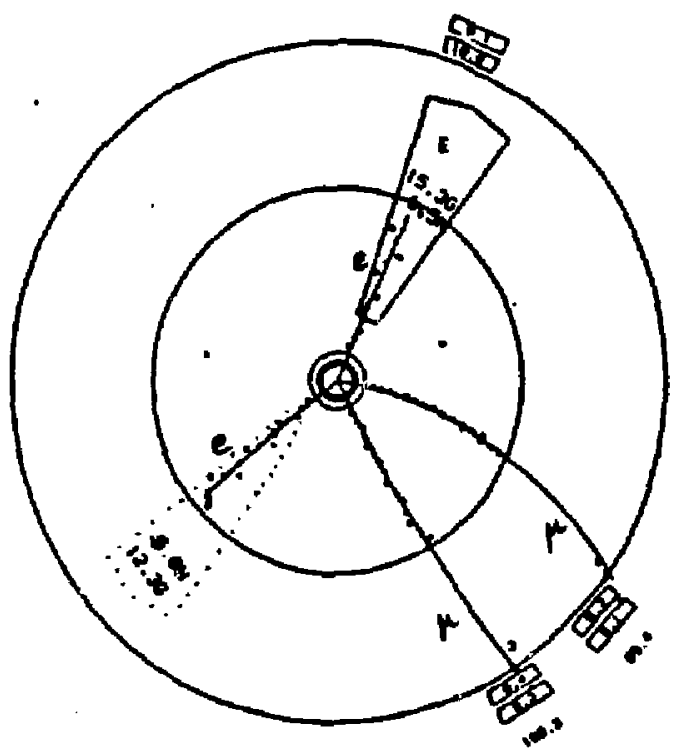

H R S RUN-4735

EVENT. '0028

DCHITS - 74

NPRNG -

SH SUH- $0.4 \cdot 27.6$

TRACK MOHENTUH THETA

$\begin{array}{rrr}1 & 12.6 & 43.1 \\ 2 & -11.5 & 137.5 \\ 3 & -3.6 & 108.5 \\ 4 & 1.1 & 80.2\end{array}$

Figure 8.17. Charseteristic $e^{+} e^{-} \mu^{+} \mu^{-}$eveat pieture obmerved in the BRS date.

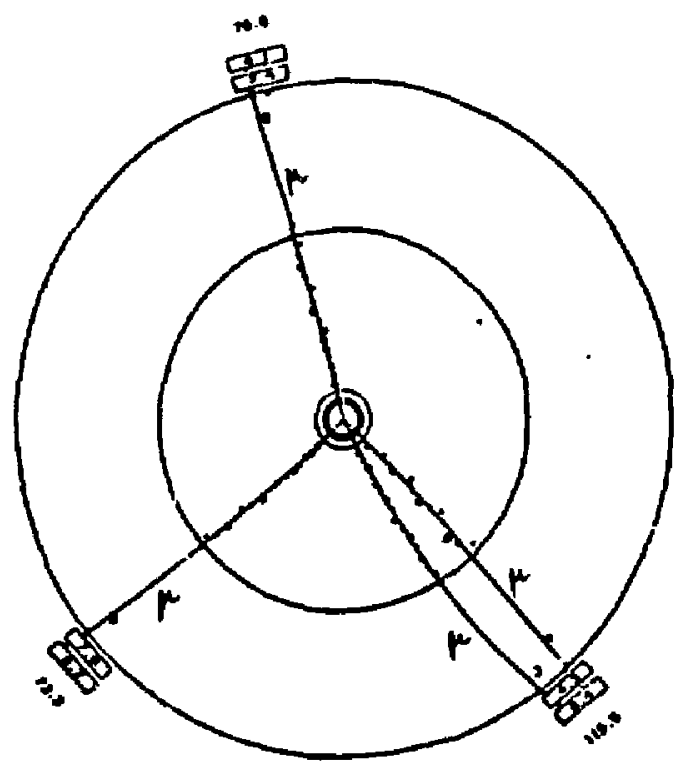

H R 5 RUN47463

EVENT - 12100

DCHITS - 109

NPRNG - 5

SH SUM. $0.8 \cdot 0.0$

TRACK MOHEHTUH THETA

$\begin{array}{rrr}2 & 5.4 & 73.5 \\ 3 & -2.3 & 103.3 \\ 4 & 0.3 & 125.8 \\ 5 & .12 .2 & 68.5\end{array}$

Figute 8.18. Picture of $\mu^{+} \mu^{-} \mu^{+} \mu^{-}$event oberved in the BRS data. 


\subsection{Detection efflciencies}

We applied kinematical cuts, before the detector simulation, on the Monte Carlo generated four-momenta files, for each of the three processes $e^{+} e^{-} e^{+} e^{-}, e^{+} e^{-} \mu^{+} \mu^{-}$ and $\mu^{+} \mu^{-} \mu^{+} \mu^{-}$. These cuts were jdentical to those used in section 8.1. In detail, each event should satisfy the following criteria :

1. there should be 4 charged leptons with zero net total charge, (true by default)

2. each lepton should have a momentum $P \geq 0.6 \mathrm{GeV} / \mathrm{c}$ and $|\cos \theta| \leq 0.9$

3. the energy $\left(\sum_{i=1,4} c\left|P_{i}\right|\right)$ of all four leptons should be $\geq 20 \mathrm{GeV}$, but $\leq 40 \mathrm{GeV}$

4. all opposite-charge pairs of leptons should have an invariant mass $\geq 1 \mathrm{GeV} / \mathrm{c}^{2}$

Table 8.4 lists the numbers of expected signal events that were obtained by applying the above requirements, both before and after detector simulation, scaled down to the HRS integrated luminosity $L$ of $291 \mathrm{pb}^{-1}$. Note that $\mathrm{N}_{\text {after refers }}$ to the final results obtained after event identification, shown already in table 8.3 for $e^{+} e^{-} e^{+} e^{-}, e^{+} e^{-} \mu^{+} \mu^{-}$and $\mu^{+} \mu^{-} \mu^{+} \mu^{-}$. The errors attached are due to limited Monte Carlo statistics. The numbers corresponding to the MC generation luminosities, $1598 p^{-1}$ of $e^{+} e^{-} e^{+} e^{-}, 1590 p b^{-1}$ of $e^{+} e^{-} \mu^{+} \mu^{-}$, and $2059 p b^{-1}$ of $\mu^{+} \mu^{-} \mu^{+} \mu^{-}$, are given in parentheses. The detection efficiencies, determined from Monte Carlo, $\epsilon=\frac{N_{\text {after }}}{N_{\text {bcforc }}}$, for $e^{+} e^{-} e^{+} e^{--}, e^{+} e^{--} \mu^{+} \mu^{-}$and $\mu^{+} \mu^{-} \mu^{+} \mu^{-}$, are $0.65 \pm 0.03,0.77 \pm 0.03$ and $0.95 \pm 0.04$, respectively.

In the same table, we present the cross sections $\sigma_{M C}$ (in $f b, 1 f b=10^{-3} p b$ ) corresponding to the number of expected signal events for each of the 3 processes. The cross section $\sigma_{M C}$ is derived from the formula $\sigma_{M C}=\frac{N_{\text {dejore }}}{L_{M C}}$, where 
$L_{M C}$ is the generation luminosity of the process, and $\mathrm{N}_{\text {before }}$ is given in parentheses in table 8.4 .

Table 8.4. Summary of BRS crom nections $\sigma_{w c}$ and Monte Carlo event statistics (Hee (ext).

\begin{tabular}{|c|c|c|c|}
\hline & $\begin{array}{c}\sigma_{M C} \\
(\mathrm{fb})\end{array}$ & $\begin{array}{c}\mathrm{N}_{\text {before }} \\
(\mathrm{MC})\end{array}$ & $\begin{array}{c}\mathrm{N}_{\text {after }} \\
(\mathrm{MC})\end{array}$ \\
\hline$e^{+} e^{-} e^{+} e^{-}$ & 126 & $36.8 \pm 2.6(202)$ & $23.5 \pm 2.2 \quad(132)$ \\
\hline$e^{+} e^{-} \mu^{+} \mu^{-}$ & 143 & $41.7 \pm 2.8(228)$ & $31.5 \pm 2.5 \quad(176)$ \\
\hline$\mu^{+} \mu^{-} \mu^{+} \mu^{-}$ & 11 & $3.1 \pm 0.7(22)$ & $2.9 \pm 0.7 \quad(21)$ \\
\hline
\end{tabular}




\section{CHAPTER 9}

\section{CONCLUSIONS}

The theoretical predictions and measurements presented in this thesis for the production of four-iepton final states $\left(e^{+} e^{-} e^{+} e^{-}, e^{-} e^{-} \mu^{+} \mu^{-}, \mu^{+} \mu^{-} \mu^{+} \mu^{-}\right)$are in good agreement. For this analysis the leptons were all required to be at large angles. The effective mass of the lepton pairs was also required to exceed $1 \mathrm{GeV} / \mathrm{c}^{2}$. For an integrated luminosity of $205 \mathrm{pb}^{-1}$ in the M-rk II experiment, we observed $10 e^{+} e^{-} e^{+} e^{-}, 10 e^{+} e^{-} \mu^{+} \mu^{-}$and $1 \mu^{+} \mu^{-} \mu^{+} \mu^{-}$events with opposite-charge pair masses greater than $1 \mathrm{GeV} / c^{2}$, while $10.9 \pm 0.7,9.6 \pm 0.8$ and $0.76 \pm 0.07$ events were expected, respectively. In the HRS experiment, for an integrated luminosity of $291 \mathrm{pb}^{-1}$, we observed $17 e^{+} e^{-} e^{+} e^{-}, 24 e^{+} e^{-} \mu^{+} \mu^{-}$and $1 \mu^{+} \mu^{-} \mu^{+}{ }_{i^{+}}^{-}$events with opposite-charge pair masses greater than $1 \mathrm{GeV} / \mathrm{c}^{2}$, while $23.5 \pm 2.2,31.5 \pm 2.5$ and $2.9 \pm 0.7$ events were expected, respectively. The various kinematical distributions also show good overall agreement. QED, calculated to fourth order, gives a good description of the data within the overall statistical uncertainty of about $20 \%$. It is interesting to note that the cross sections tested in this thesis are at the $0.1 \mathrm{pb}$ level compared to the total annihilation cross section of the order of $100 \mathrm{pb}$.

For completeness, we summarize the basic selection criteria and results for 
large-angle $e^{+} e^{-} e^{+} e^{-}$and $e^{+} e^{-} \mu^{+} \mu^{-}$events from the CELLO, JADE, Mark J, ASP, HRS and Mark II experiments (from refs. 8, 10 and this thesis), in tables 9.1 and 9.2, respectively. The tiny $e^{+} e^{-} \rightarrow \mu^{+} \mu^{-} \mu^{+} \mu^{-}$cross section has been observed only in the CELLO, Mark II and HRS detectors. This is not surprising since these experiments have accumulated the highest integrated luminosities in $e^{+} e^{-}$storage rings in a range of c.m. energies $14-47 \mathrm{GeV}$.

Table 9.1. Summary of basic selection cuts and number of large angle $e^{+} e^{-} e^{+} e^{-}$ events from JADE, ASP, CELLO, Mark II and ZRS. $P_{e}$ is the electron( $\left(e^{ \pm}\right)$momentum, $M\left(l^{+} \gamma^{-}\right)$is the invariant mass of any opposite-charge pair of tracks.

\begin{tabular}{|c|c|c|c|c|c|}
\hline & JADE & ASP & CELLO & Mark II & HRS \\
\hline$e^{+} e^{-} e^{+} e^{-}$ & 13 & 15 & 16 & 10 & 17 \\
\hline $\int L d t\left(p^{-1}\right)$ & 95 & 109.6 & 130 & 205 & 291 \\
\hline$\left|\cos \theta_{e}\right|$ & $<0.955$ & $<0.94$ & $<0.85$ & $<0.88$ & $<0.9$ \\
\hline$P_{e}$ & $>E_{\text {beam }} / 3$ & $>0.3 \mathrm{GeV}$ & $>0.05 * E_{\text {beam }}$ & $>1 \mathrm{GeV}$ & $>1 \mathrm{GeV}$ \\
\hline$M\left(l^{+} l^{-}\right)$ & - & $>0.5 \mathrm{GeV}$ & $>0.01 \mathrm{GeV}$ & $>1 \mathrm{GeV}$ & $>1 \mathrm{GeV}$ \\
\hline
\end{tabular}


Table 9.2. Summary of batic selection euts and number of large angle $e^{+} e^{-} \mu^{+} \mu^{-}$events from JADE, Mark J, CELLO, Mark II and BRS. $P_{\text {q }}$ is the electron( $e^{ \pm}$) momeatum, - $M\left(1^{+} I^{-}\right)$is the invariant mus of any oppoeite-cherge pair of tracks, $P_{\mu}$ is the muon $\left(\mu^{ \pm}\right)$ momentum.

\begin{tabular}{|c|c|c|c|c|c|}
\hline & JADE & Mark J & CELLO & Mark II & HRS \\
\hline$e^{+} e^{-} \mu^{+} \mu^{-}$ & 8 & 9 & 8 & 10 & 24 \\
\hline $\int L d t\left(p b^{-1}\right)$ & 95 & 113 & 130 & 205 & 291 \\
\hline$\left|\cos \theta_{e}\right|$ & $<0.955$ & $<0.94$ & $<0.85$ & $<0.88$ & $<0.9$ \\
\hline$P_{e}$ & $>E_{\text {beam }} / 3$ & $>0.2 * E_{\text {beam }}$ & $>0.05 * E_{\text {beam }}$ & $>1 \mathrm{GeV}$ & $>1 \mathrm{GeV}$ \\
\hline$\left|\cos \theta_{\mu}\right|$ & $<0.966$ & $<0.87$ & $<0.92$ & $<0.55$ & $<0.9$ \\
\hline$P_{\mu}$ & $>1 \mathrm{GeV}$ & $>1.5 \mathrm{GeV}$ & $>0.1 * E_{\text {beam }}$ & $>1 \mathrm{GeV}$ & $>1 \mathrm{GeV}$ \\
\hline$M\left(l^{+} l^{-}\right)$ & - & $>1 \mathrm{GeV}$ & $>0.01 \mathrm{GeV}$ & $>1 \mathrm{GeV}$ & $>1 \mathrm{GeV}$ \\
\hline
\end{tabular}




\section{APPENDIX A}

\section{RADIATIVE CORRECTIONS}

A number of papers have been devoled to the problem of radiative corrections to processes of the type $e^{+} e^{-} \rightarrow e^{+} e^{-} e^{+} e^{-}, e^{+} e^{-} \mu^{+} \mu^{-}$, bu ${ }^{+}$unfortunately not in our domain of interest, where the leptons are emitted at large angles. They all consider the case where both of the outgoing electrons are either not detected, or detected at small angles. (13-45) Even Berends et al., whose MC programs were used to generate fourth order QED events, have only treated the radiative corrections for multipesipheral four-lepton production, ${ }^{(30)}$ which peaks at small angles.

The emission of a real or virtual photon from the electron or positron before they annihilate is called initial-state radiation. It is known from QED that a fast, interacting particle can emit radiation (bremsstrahlung) into a narrow cone in the direction of its motion. Scattering cross sections are proportional to $1 / \mathrm{S}$, where $S$ is the center-of-mass (c.m.) energy squared of the incor $: g$ particles. Since the c.m. energy of the incoming particles may decrease due to bremsstrahlung, the cross section may change. This change due to initial-state radiation needs to be taken into account in measurements of cross sections. Final-state radiation, emitted after the annihilation process, does not have a similar impact on the measured cross sections. 
We now present an eatimate of the magnitude and sign of the correction for initial-state radiation. We need only consider collinear radiation, since photon emission peaks in directions parallel to the motion of the charged particlea.

The state of the art in radiative corrections using renormalization group methods is found in the paper by Kursev and Fadin. ${ }^{(10)}$ They consider the radiative corrections to single-photon annihilation of an $e^{+} e^{-}$pair that are only associated with the initial-state. These radiative corrections are part of the corrections to all processes with an arbitrary final state. We make use of their probability function $F(x, S)$ to emit photons which carry away a fraction $x$ of the beam energy. The proper definition of $x$ is $1-S^{\prime} / S$, where $S$ is the nominal and $S^{\prime}$ the observed c.m. energy squared.

The aspect of initial-state radiation that is most important for the four-lepton study is the nongaussian c.m. energy distribution of the incoming leptons, and in particular the long tail to lower energies shown in fig. A.1. The cutoff at $16 \mathrm{GeV}$ is well below the cut spplied on the energy (total scalar momentum) of the data sets (see sections $7.1,8.1$ ).

We now present our methodology:

1. We generate $\mathrm{MC}^{+} e^{-} e^{+} e^{-}$events at various energies $\mathrm{E}^{\prime}$ below the nominal $E=29 \mathrm{GeV}$, with enough statistics to keep the statistical error arising from MC limited statistics small. We assume a photon of energy $\mathbf{E}_{\boldsymbol{\gamma}}$ is emitted from one of the beam electrons along $z$, thus reducing the c.m. energy from $E$ to $E^{\prime}=E-E_{y}$. Figure A.2 shows the generated cross section before any 


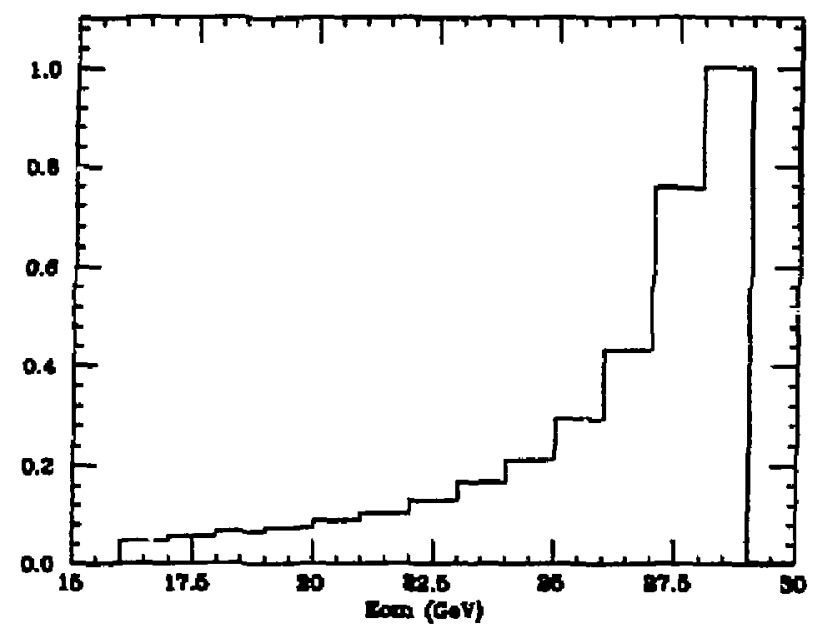

Figure A.1. The center-of-man energy dintritution of Monte Carlo events afler initialatate radiation of a beam with nominal center-of-mese energy equal to $28 \mathrm{GeV}$ and sigma equal to $17 \mathrm{MeV}$.

cuts sre applied. The procedure followed in the generation of $\mathrm{e}^{+} e^{-} e^{+} e^{-}$is presented here : we first transform all quantities relevart to the generation ( $E_{\text {beam }}, \theta_{\min }, \theta_{\max }$ of electrons) from the laboratory frame after radiation $\left(E^{\prime}, 18.5^{\circ}, 161.5^{\circ}\right.$ electron angles) to a new c.m. frame, where we let the MC generator produce four-momenta of the four-lepton events. Then we transform back to the lab, where we apply a crude set of kinematical cuts on the generated fcur-momenta, identical to the ones described in chapter 5 . The parameters used to generate the data are listed in table A.1 .

2. Then we pass those four-momenta through the Mark 11 detector simulation Monte Carlo programs and through our analysis cuts, previously described in section 7.1. This way, for every energy $E^{\prime}$ we get a corresponding observed cross section. We then fit the points (cross section, $E^{\prime}$ ) to an empirically found smooth function of the form $(1+\tanh (\alpha \mathrm{E}-\beta))(c+\mathrm{dE})$ where $\alpha, \beta, c, d$ 
109

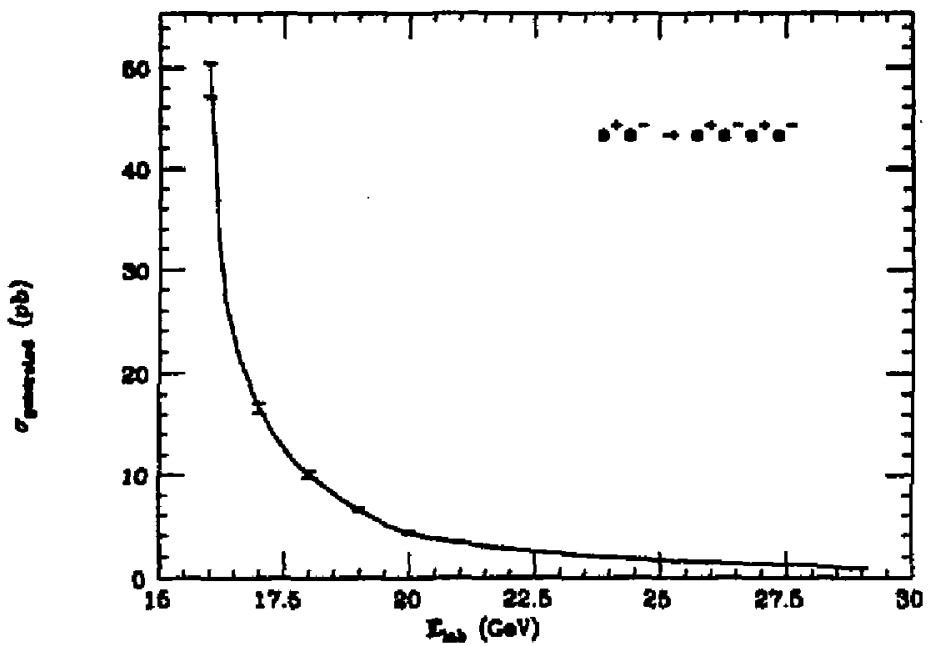

Figure A.2. The distribution of MC generated croes section at various lab energies.

are parameters determined from the fit. Figure A.3 shows the observed cross section and the empirical function which fits the points.

3. Using Simpson's rule over small enough energy intervals $(0.1 \mathrm{GeV})$, we integrate numerically the cross sectional function we found, weighted by the Kuraev-Fadin probability function $F(x, S)$.

The result of the numerical integration is our radiatively corrected cross section.

To determine the uncertainty of the corrected cross section :

1. We varied every parameter (one at a time) of the cross sectional function by one standard deviation of the parameter value, and found the corresponding variance of the result of the integration.

2. The variance of the corrected cross section is simply the sum of the corresponding variances.

We find that the cross section of $e^{+} e^{-} \rightarrow e^{+} e^{-} e^{+} e^{-}$, corrected for initial-state radiation, is $(0.058 \pm 0.004) \mathrm{pb}$, whereas the observed is $(0.059 \pm 0.003) \mathrm{pb}$. There- 
Table A.1. Liat of the parameters uned to gederale Monte Carlo $t^{+} e^{-t_{e}-\text { events at }}$ . variout lab energieu $\mathbf{E}^{\prime}$.

\begin{tabular}{|c|c|c|c|c|}
\hline $\mathrm{E}^{\prime}$ & $\theta_{\mathrm{cm} / \mathrm{lab}}$ & $\begin{array}{c}\mathrm{E}_{\text {beam }} \\
\mathrm{CM}\end{array}$ & $\begin{array}{c}\theta_{\min } \\
\mathrm{CM}\end{array}$ & $\begin{array}{c}\theta_{\max } \\
\mathrm{CM}\end{array}$ \\
\hline $\mathrm{GeV}$ & $-1 / 28$ & 13.99 & $17.9^{\circ}$ & $163^{\circ}$ \\
\hline 27 & $-2 / 27$ & 13.46 & $17.2^{\circ}$ & $160^{\circ}$ \\
\hline 26 & $-3 / 26$ & 12.91 & $16.6^{\circ}$ & $159^{\circ}$ \\
\hline 25 & $-4 / 25$ & 12.34 & $15.8^{\circ}$ & $158^{\circ}$ \\
\hline 24.5 & $-4.5 / 24.5$ & 12.04 & $15.4^{\circ}$ & $157.8^{\circ}$ \\
\hline 24 & $-5 / 24$ & 11.7 & $15.1^{\circ}$ & $157^{\circ}$ \\
\hline 23.5 & $-5.5 / 23.5$ & 11.42 & $14.6^{\circ}$ & $156.6^{\circ}$ \\
\hline 22 & $-7 / 22$ & 10.43 & $13.5^{\circ}$ & $154^{\circ}$ \\
\hline 21 & $-8 / 21$ & 9.71 & $13^{\circ}$ & $152^{\circ}$ \\
\hline 20 & $-9 / 20$ & 8.93 & $12^{\circ}$ & $150^{\circ}$ \\
\hline 19 & $-10 / 19$ & 8.08 & $10.4^{\circ}$ & $147.4^{\circ}$ \\
\hline 18 & $-11 / 18$ & 7.12 & $9.2^{\circ}$ & $143.3^{\circ}$ \\
\hline 17 & $-12 / 17$ & 6.02 & $8^{\circ}$ & $137^{\circ}$ \\
\hline 16 & $-13 / 16$ & 4.66 & $6^{\circ}$ & $126^{\circ}$ \\
\hline
\end{tabular}

fore we estimate that an approximate overall correction, defined as the ratio of the corrected $e^{+} e^{-} \rightarrow e^{+} e^{-} e^{+} e^{--}$cross section over the observed $e^{+} e^{-} \rightarrow e^{+} e^{-} e^{+} e^{-}$ cross section, is $0.98 \pm 0.08$. The set of points used in the fit to the empirically found function are presented in table A.2, whereas table A.3 shows the $M C$ rates and efficiencies at various lab energies $E^{\prime}$. We define the efficiency $R$ at each $E^{\prime}$ (shown in fig. A.4) as the ratio of the number of $\mathrm{MC}$ simulated events which survive the 
111

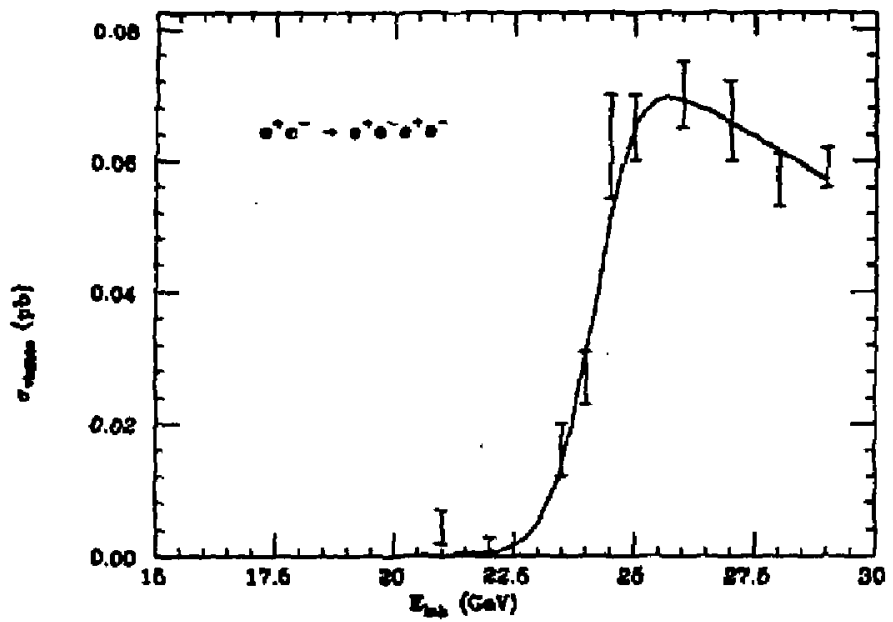

Figure A.3. The distribution of MC obeerved cras section at various lab energies.

analysis cuts over the number of the corresponding $M C$ generated events at the specific $E^{\prime}$.

The vanishing of the observed cross section below $21 \mathrm{GeV}$ is the result of the combination of mainly two effects. First, the applied boost from the laboratory after radiation to the $\mathrm{MC}$ c.m. Frame, where we generated our data at every $E^{\prime}$, causes a shrinking of the effective cone in which the four leptons are produced. The magnitude of the emitted photon momentum determines the relative speed $\beta_{\text {en/lab }}$ between the lab frame and the c.m. frame of the theoretical MC. Note that the lab frame had initially zero momentum before the emission of the ph con. The higher this relative speed is, the more pronounced the relativistic 'beadlight' effect becomes. In othet words, large angles in the MC frame become substantially smaller after their transformation to the lab frame, for large relative speeds. The steep fall at around $24 \mathrm{GeV}$ of the visible cross section curve comes from a drastic reduction of events with four good tracks. The second effect (weaker than the first) comes 
Table A.2. Generated and vinible croas actions (in picobarns) at various lab energies $E^{\prime}$ of Monte Carlo ete- $e^{+} e^{-}$evento.

\begin{tabular}{|c|c|c|}
\hline $\begin{array}{c}\mathbf{E}^{\prime} \\
\mathrm{GeV}\end{array}$ & $\begin{array}{c}\sigma_{g e n} \pm \delta \sigma_{g e n} \\
\mathrm{pb}\end{array}$ & $\begin{array}{c}\sigma_{v i s} \pm \delta \sigma_{\text {vis }} \\
\mathrm{pb}\end{array}$ \\
\hline 29 & $0.88 \pm 0.01$ & $0.059 \pm 0.003$ \\
\hline 28 & $1.13 \pm 0.02$ & $0.057 \pm 0.004$ \\
\hline 27 & $1.28 \pm 0.02$ & $0.066 \pm 0.006$ \\
\hline 26 & $1.44 \pm 0.02$ & $0.070 \pm 0.005$ \\
\hline 25 & $1.68 \pm 0.03$ & $0.065 \pm 0.005$ \\
\hline 24.5 & $1.82 \pm 0.03$ & $0.062 \pm 0.008$ \\
\hline 24 & $1.95 \pm 0.03$ & $0.027 \pm 0.004$ \\
\hline 23.5 & $2.15 \pm 0.04$ & $0.016 \pm 0.004$ \\
\hline 22 & $2.79 \pm 0.05$ & $0.0014 \pm 0.0014$ \\
\hline 21 & $3.34 \pm 0.06$ & $0.0043 \pm 0.0025$ \\
\hline 20 & $4.32 \pm 0.10$ & $<0.04 @ 95 \% \mathrm{CL}$ \\
\hline 19 & $6.58 \pm 0.18$ & $<0.06 @ 95 \% \mathrm{CL}$ \\
\hline 18 & $10.11 \pm 0.32$ & $<0.20 @ 95 \% \mathrm{CL}$ \\
\hline 17 & $16.64 \pm 0.50$ & $<0.16 @ 95 \% \mathrm{CL}$ \\
\hline 16 & $48.78 \pm 1.71$ & $<0.49 @ 95 \% \mathrm{CL}$ \\
\hline
\end{tabular}

from applying a $1 \mathrm{GeV} / \mathrm{c}^{2}$ invariant mass cut on all zero charge pairs of two tracks. The lower the c.m. energy, the harder the above mass cut becomes.

In conclusion, we have seen that initial-state radiation, collinear to the incoming electrons, does not affect significantly the Born level cross section of $e^{+} \epsilon^{-} \rightarrow$ $e^{+} e^{-} e^{+} e^{-}$, or any other four lepton process in which the four leptons are detected at large angles, high $Q^{2}$. 
Table A.3. Generated and visible rates and efficiencies at various lab energies $E^{\prime}$ of . Monte Carlo $e^{+} e^{-} e^{+} e^{-}$events.

\begin{tabular}{|c|c|c|c|}
\hline $\begin{array}{c}\mathrm{E}^{\prime} \\
\mathrm{GeV}\end{array}$ & $\mathrm{N}_{\text {gen }}$ & $\mathrm{N}_{\text {vis }}$ & $\mathrm{R} \pm \delta \mathrm{R}$ \\
\hline 29 & 5284 & 360 & $0.068 \pm 0.003$ \\
\hline 28 & 3742 & 187 & $0.050 \pm 0.004$ \\
\hline 27 & 2916 & 153 & $0.052 \pm 0.004$ \\
\hline 26 & 4243 & 205 & $0.048 \pm 0.003$ \\
\hline 25 & 4285 & 165 & $0.039 \pm 0.003$ \\
\hline 24.5 & 1902 & 65 & $0.034 \pm 0.004$ \\
\hline 24 & 3312 & 47 & $0.014 \pm 0.002$ \\
\hline 23.5 & 2756 & 21 & $0.008 \pm 0.002$ \\
\hline 22 & 2040 & 1 & $0.0005 \pm 0.0005$ \\
\hline 21 & 2270 & 3 & $0.0013 \pm 0.0008$ \\
\hline 20 & 188 & 0 & $<0.01995 \% \mathrm{CL}$ \\
\hline 19 & 183 & 0 & $<0.01095 \% \mathrm{CL}$ \\
\hline 18 & 166 & 0 & $<0.02995 \% \mathrm{CL}$ \\
\hline 17 & 185 & 0 & $<0.01995 \% \mathrm{CL}$ \\
\hline 16 & 179 & 0 & $<0.01 @ 95 \% \mathrm{CL}$ \\
\hline
\end{tabular}


114

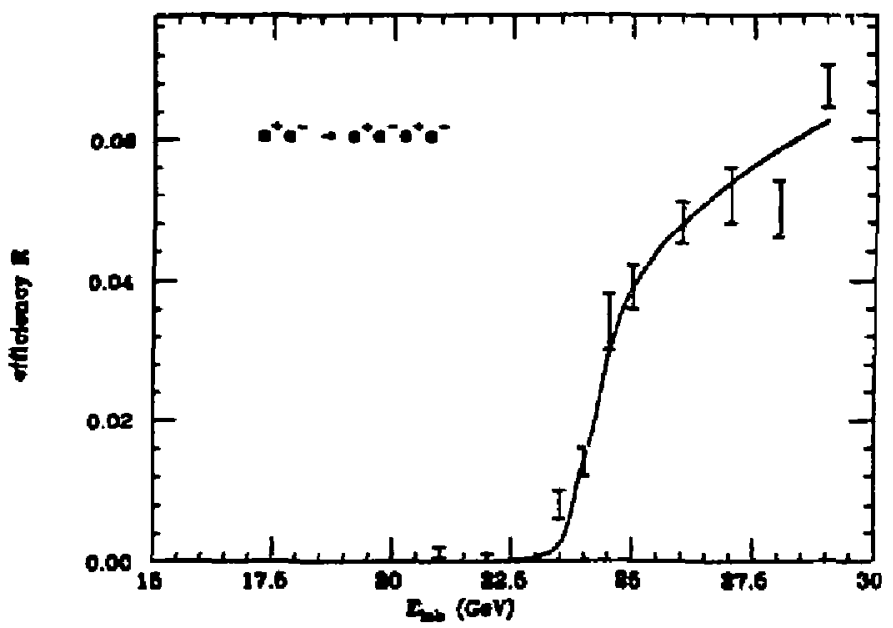

Figure A.4. The diatribution of MC efficiency at various lab energies. 


\section{APPENDIX B}

\section{STUDY OF THE MARK II END CAP SHOWER COUNTER}

We have investigated the use of the Mark II/PEP end cap shower counter (EC) in order to differentiate electrons $\left(e^{ \pm}\right)$from muons $\left(\mu^{ \pm}\right)$in four-lepton candidate events. We do not worry about other interacting particles, such as pions, since we found that the background to the four-lepton processes that interest us $\left(e^{+} e^{-} \rightarrow e^{+} e^{-} e^{+} e^{-}, e^{+} e^{-} \mu^{+} \mu^{-}, \mu^{+} \mu^{-} \mu^{+} \mu^{-}\right)$is very small (see tables 7.5 and 7.6).

We used radiative Bhabbas, hadronic and cosmic ray data, in order to study the EC performance when it is bit by electrons and minimum ionizing particles. Electrons hitting the EC were selected among $e^{+} e^{-} \gamma$ events ${ }^{(* 8)}$ that came from the total sample of Mark II/PEP data, in accordance with the following requirements:

1. 2 charged tracks.

2. One neutral energy cluster with energy of at least $1 \mathrm{GeV}$, al least $20 \mathrm{~cm}$ away from a charged track at the liquid argon calorimeter (LA) entrance.

3. the sum of the scalar momenta of the 2 charged tracks and of the photon energy should be at least $24 \mathrm{GeV}$.

4. at least one good electron. An electron is good if it has momentum greater 
than $1 \mathrm{GeV} / \mathrm{C}$ and if the ratio $\mathrm{E} / \mathrm{P}$ of the energy $\mathrm{E}$ deposited in the $\mathrm{L} A$ over : its momentum $\mathrm{P}$ measured by the drift chamber is greater than 0.7 .

Forty-two $e^{+} e^{-} \gamma$ were selected for our study. In these events, one of the electrons had an equivalent $E / P>0.6$, while the photon hit the $L A$. The other electron was going into the fiducial volume of the EC, avoiding the azimuthal region where lies a keyway cut : $4.2 \leq \phi \leq 5.2$. We explicitly demanded that the angular range of those EC electrons be $0.76 \leq|\cos \theta| \leq 0.85$, as measured by the drift chamber, in order to ensure a good momentum measurement.

Based on these $42 e^{+} e^{-\gamma}$ events, we present fig. B.1 to show the EC response to electrons. The energy deposited by the electrons in the EC is shown in fig. (a). The distribution of $\mathrm{E} / \mathrm{P}$ is shown in fig. (b).
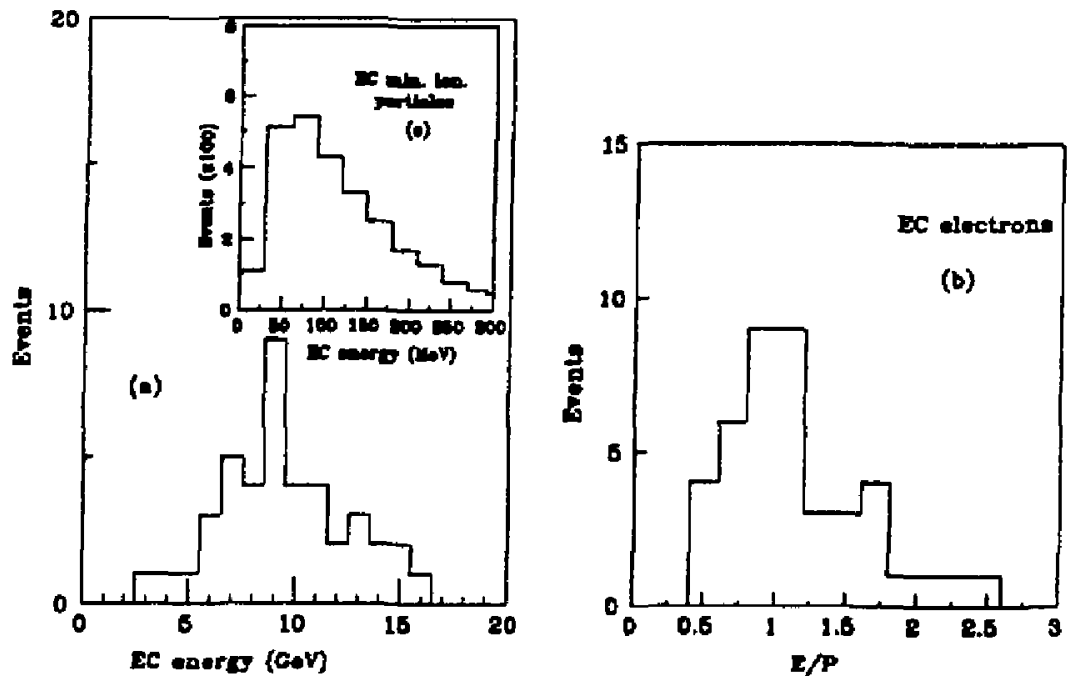

Figure B.1. Energy deposited in the end cap from (a) electrons and (c) minimum ionizing purticles; the ratio E/P for electrons is shown in (b) (we text). 
For hadronic events with at least 4 charged tracks, and with momenta of at least $3 \mathrm{GeV} / \mathrm{c}$, we present the distribution of minimum ionizing particles hitting the EC in the inset fig. (c).

Cosmic ray data taken at PEP have also shown that, for EC tracks with $0.76 \leq|\cos \theta| \leq 0.85$, the average pulse height in the EC, multiplied by $\cos \theta$, is $105 \mathrm{MeV}$.

After taking into consideration the above presented distributions, we decided to use the EC information to distinguish electrons from muons in four-lepton candidate events. Tracks with $0.76 \leq|\cos \theta| \leq 0.85$ and momenta of at least $3 \mathrm{GeV} / \mathrm{c}$ are identified as electrons, as long as they have $E / P>0.5$. If they have $E / P \leq 0.5$, they are considered to be muons or hadrons. 


\section{APPENDIX C}

\section{MARK II EVENT LIST}

Table C.l contains the list of the 38 four-lepton candidate events observed in the Mark II data, where all four tracks are detected at large angles, and have oppositecharge pair masses $\geq 1 \mathrm{GeV} / \mathrm{c}^{2}$. The energy of an event is defined as the sum of the scalar momenta times $c, \sum_{i=1,4} c\left|P_{i}\right|$. The smallest mass of an opposite-charge pair of tracks in an event is denoted by $M_{\min }$. The identities of tracks with momentum $\mathrm{P} \geq 1 \mathrm{GeV} / \mathrm{c}$ are categorized as follows :

- $2=$ 'electron' : tracks in the fiducial volume of the liquid argon calorimeter and $E / P>0.6$ (where $E$ is the energy measured in the calorimeter); or tracks in the fiducial volume of the end cap shower counter with $P \geq 3 \mathrm{GeV} / \mathrm{c}$ and $\mathrm{E} / \mathrm{P}>0.5$.

- $3=$ 'muon' : tracks identified by muon system.

- 4 = 'hadron' (='not electron' and 'not muon') : tracks pointing into the fiducial volume of the calorimeters and the muon system, but which are not identified as either electrons or muons.

- $5=$ ambiguous ('electron of hadron') : tracks pointing into the muon sy'stem and not identified muons, but for which the information from the 
calorimeters is insufficient for allowing electron identification.

- 6 = ambiguous ('muon or hadron') : tracks pointing into the calorimeters and not identified as electrons, but for which the muon system information is insufficient for allowing muon identification.

- 7 = ambiguous ('electron or muon or hadron') : all tracks with $\mathrm{P} \geq 1 \mathrm{GeV} / \mathrm{c}$ which are not in the above categories.

- $8=$ tracks with $\mathrm{P}<1 \mathrm{GeV} / \mathrm{c}$ for which no identification is attempted.

Note that in the codes 5-7, the particle identity is ambiguous because the track enters regions not well covered by the calorimeters or by the muon system. 
Table C.1. Mark If four-lepton candjdate evento with pair mawes $\geq 1 \mathrm{GeV} / \mathrm{c}^{2}$.

\begin{tabular}{|c|c|c|c|c|}
\hline Run\# Rec\# & $\begin{array}{c}\text { Energy } \\
\text { GeV }\end{array}$ & $\begin{array}{c}M_{\min } \\
\mathrm{GeV} / \mathrm{c}^{2}\end{array}$ & Track ID & Event ID \\
\hline 82823319 & 35.2 & 1.03 & 7787 & \\
\hline 88625656 & 27.9 & 5.41 & 2737 & \\
\hline 95023048 & 23.5 & 2.68 & 8222 & $e^{+} e^{-} e^{+} e^{-}$ \\
\hline 96991311 & 21.7 & 4.42 & 2277 & \\
\hline 98356825 & 28.6 & 2.98 & 7733 & \\
\hline 99025972 & 29.3 & 8.17 & 5272 & $e^{+} e^{-} e^{+} e^{-}$ \\
\hline 99287987 & 28.7 & 3.96 & 2328 & $e^{+} e^{-} \mu^{+} \mu^{-}$ \\
\hline 999512751 & 23.2 & 1.69 & 6627 & \\
\hline 100444441 & 29.5 & 4.22 & 3227 & $e^{+} e^{-} \mu^{+} \mu^{-}$ \\
\hline 1012910891 & 30.7 & 5.93 & 2222 & $e^{+} e^{-} e^{+} e^{-}$ \\
\hline 102822028 & 24.6 & 4.57 & 7226 & \\
\hline 104083884 & 26.9 & 1.18 & 8223 & $e^{+} e^{-} \mu^{+} \mu^{-}$ \\
\hline 1040811257 & 32.4 & 3.57 & 2277 & \\
\hline 104451369 & 23.2 & 1.48 & 8778 & \\
\hline 104918818 & 27.2 & 3.74 & 7227 & \\
\hline 105614044 & 34.8 & 2.49 & 8222 & $e^{+} e^{-} e^{+} e^{-}$ \\
\hline 105884966 & 28.3 & 2.44 & 5737 & \\
\hline 105916637 & 30.8 & 1.33 & 2673 & $e^{+} e^{-} \mu^{+} \mu^{-}$ \\
\hline 109433194 & 24.5 & 4.78 & 3336 & $\mu^{+} \mu^{-} \mu^{+} \mu^{-}$ \\
\hline 111027798 & 29.5 & 1.18 & 2222 & $e^{+} e^{-} e^{+} e^{-}$ \\
\hline
\end{tabular}


: Table C.1. Mark 11 four-lepton candidate events with pair mame $\geq 1 \mathrm{GeV} / \mathrm{C}^{2}$ (cont.)

\begin{tabular}{|c|c|c|c|c|}
\hline Run\# Rec\# & $\begin{array}{c}\text { Energy } \\
\text { GeV }\end{array}$ & $\begin{array}{c}M_{\min } \\
\mathrm{GeV} / \mathrm{c}^{2}\end{array}$ & Track ID & Event ID \\
\hline 11167881 & 26.1 & 1.72 & 2233 & $e^{+} e^{-} \mu^{+} \mu^{-}$ \\
\hline 11215785 & 28.9 & 2.76 & 3232 & $e^{+} e^{-} \mu^{+} \mu^{-}$ \\
\hline 112202848 & 30.1 & 6.27 & 2227 & $e^{+} e^{-} e^{+} e^{-}$ \\
\hline 112434233 & 26.8 & 4.92 & 2332 & $e^{+} e^{-} \mu^{+} \mu^{-}$ \\
\hline 113031949 & 28.9 & 1.98 & 6782 & \\
\hline 1131317720 & 21.2 & 2.66 & 3823 & $e^{+} e^{-} \mu^{+} \mu^{-}$ \\
\hline 115993628 & 23.3 & 1.70 & 6822 & \\
\hline 117474273 & 27.9 & 3.39 & 8322 & $e^{+} e^{-} \mu^{+} \mu^{-}$ \\
\hline 117949896 & 23.2 & 2.16 & 2222 & $e^{+} e^{-} e^{+} e^{-}$ \\
\hline 119122616 & 21.9 & 2.50 & 5222 & $e^{+} e^{-} e^{+} e^{-}$ \\
\hline 1196111314 & 29.1 & 1.39 & 8227 & \\
\hline 122751442 & 28.5 & 4.39 & 5227 & \\
\hline 122907874 & 29.3 & 2.06 & 2227 & $e^{+} e^{-} e^{+} e^{-}$ \\
\hline 124187208 & 27.5 & 2.35 & 2277 & \\
\hline 125137732 & 27.7 & 1.79 & 2527 & \\
\hline 1275612730 & 28.2 & 1.02 & 6672 & \\
\hline 129373891 & 27.9 & 2.78 & 3322 & $e^{+} e^{-} \mu^{+} \mu^{-}$ \\
\hline 1319315156 & 28.2 & 1.68 & 2222 & $e^{+} e^{-} e^{+} e^{-}$ \\
\hline
\end{tabular}




\section{APPENDIX D}

\section{HRS EVENT LIST}

Table D.1 contains the list of the 73 four-lepton candidate events observed in the HRS data, where all four tracks are detected at large angles, and bave oppositecharge pair masses $\geq 1 \mathrm{GeV} / \mathrm{c}^{2}$. The energy of an event is defined as the sum of the

scalar momenta times $\mathrm{c}, \sum_{i=2,4} c\left|P_{i}\right|$. The smallest mass of an opposite-charge pair of tracks in an event is denoted by $M_{\min }$. The identities of tracks with momentum $P \geq 1 \mathrm{GeV} / \mathrm{c}$ are categorized as follows :

- 2 = 'electron' : tracks with $0.55<\mathrm{E} / \mathrm{P}<1.45$, where $\mathrm{E}$ is the energy measured in the calorimeters.

- $3=$ 'muon': tracks in the fiducial volume of calorimeters and $0<E<0.5 \mathrm{GeV}$.

- 4 = 'hadron' (='not electron' and 'not muon') : tracks in the fiducial volume of calorimeters with $E / P \leq 0.55$ and $E \geq 0.5 \mathrm{GeV}$.

- 5 = ambiguous ('electron or hadron') : tracks with $\mathrm{E} / \mathrm{P} \leq 0.55$ and $\mathrm{E} \geq 0.5 \mathrm{GeV}$, but which are not in the fiducial volume of the calorimeters.

- $7=$ smbiguous ('electron or muon or hadron') : tracks with $\mathrm{E} / \mathrm{P} \leq 0.55$ and $0<\mathrm{E}<0.5 \mathrm{GeV}$ which are not in the fiducial volume of the calorimeters.

- $8=$ tracks with $P<1 \mathrm{GeV} / \mathrm{c}$ for which no identification is attempted. 
- $9=$ tracks with $\mathrm{E} / \mathrm{P}>1.45$.

- $0=$ no energy deposit was associated with the tracks (tracks miss calorimeters).

Table D.1. BRS four-lepton candidate events with pair manes $\geq 1 \mathrm{GeV} / \mathrm{c}^{2}$.

\begin{tabular}{|c|c|c|c|c|}
\hline Run\# Rec\# & $\begin{array}{c}\text { Energy } \\
\mathrm{GeV}\end{array}$ & $\begin{array}{c}M_{\min } \\
\mathrm{GeV} / \mathrm{c}^{2}\end{array}$ & Track ID & Event ID \\
\hline 115720210 & 27.4 & 2.47 & 8000 & \\
\hline 123918757 & 20.5 & 1.05 & 2200 & \\
\hline 123922262 & 29.1 & 2.16 & 2270 & \\
\hline 123925671 & 26.6 & 1.28 & 0732 & \\
\hline 26485063 & 21.3 & 1.46 & 0000 & \\
\hline 38206537 & 29.7 & 1.44 & 2230 & $e^{+} e^{-} \mu^{+} \mu^{-}$ \\
\hline 39514717 & 28.3 & 9.50 & 3702 & \\
\hline 39538637 & 34.3 & 1.04 & 3333 & \\
\hline 400016596 & 31.3 & 1.20 & 2732 & $e^{+} e^{-} \mu^{+} \mu^{-}$ \\
\hline 404812168 & 21.9 & 1.28 & 8222 & $e^{+} e^{-} e^{+} e^{-}$ \\
\hline 407718840 & 27.4 & 4.15 & 3302 & $e^{+} e^{-} \mu^{+} \mu^{-}$ \\
\hline 407816596 & 26.7 & 4.96 & 2022 & $e^{+} e^{-} e^{+} e^{-}$ \\
\hline 410619452 & 28.9 & 7.83 & 0502 & \\
\hline 41732809 & 29.1 & 4.33 & 2222 & $e^{+} e^{-} e^{+} e^{-}$ \\
\hline 12491228 & 21.1 & 1.86 & 8330 & \\
\hline 427241056 & 29.1 & 1.78 & 2072 & $e^{+} e^{-} e^{+} e^{-}$ \\
\hline 43766575 & 20.6 & 1.32 & 4322 & \\
\hline 43942556 & 27.5 & 3.47 & 3003 & \\
\hline
\end{tabular}


$\therefore$ Table D.1. BRS four-lepton candidate events with pair maser $\geq 1 \mathrm{GeV} / \mathrm{c}^{2}$ (cont.)

\begin{tabular}{|c|c|c|c|c|}
\hline Run\# Rec\# & $\begin{array}{c}\text { Energy } \\
\text { GeV }\end{array}$ & $\begin{array}{c}M_{\min } \\
\mathrm{GeV} / \mathrm{c}^{2}\end{array}$ & Track ID & Event ID \\
\hline 45215233 & 29.5 & 4.97 & 2502 & $e^{+} e^{-} e^{+} e^{-}$ \\
\hline 453034503 & 22.7 & 1.49 & 2332 & $e^{+} e^{-} \mu^{+} \mu^{-}$ \\
\hline 45534660 & 29.1 & 1.90 & 2302 & $e^{+} e^{-} \mu^{+} \mu^{-}$ \\
\hline $4560 \quad 10292$ & 23.0 & 1.84 & 3722 & $e^{+} e^{-} \mu^{+} \mu^{-}$ \\
\hline 47237680 & 28.6 & 1.14 & 8222 & $e^{+} e^{-} e^{+} e^{-}$ \\
\hline 47359928 & 29.0 & 2.01 & 2237 & $e^{+} e^{-} \mu^{+} \mu^{-}$ \\
\hline 474626595 & 28.6 & 1.27 & 2222 & $e^{+} e^{-} e^{+} e^{-}$ \\
\hline 49221498 & 27.2 & 5.90 & 7322 & $e^{+} e^{-} \mu^{+} \mu^{-}$ \\
\hline 500617477 & 25.2 & 6.40 & 0242 & \\
\hline 516920394 & 24.2 & 1.25 & 2220 & $e^{+} e^{-} e^{+} e^{-}$ \\
\hline 521123666 & 27.9 & 1.50 & 0222 & $e^{+} e^{-} e^{+} e^{-}$ \\
\hline 524617930 & 29.6 & 4.02 & 3202 & $e^{+} e^{-} \mu^{+} \mu^{-}$ \\
\hline 533323206 & 26.2 & 1.01 & 2273 & $e^{+} e^{-} \mu^{+} \mu^{-}$ \\
\hline 536825374 & 28.5 & 1.08 & 4202 & \\
\hline 540826072 & 28.6 & 1.38 & 0023 & \\
\hline 559411956 & 24.6 & 1.38 & 2082 & \\
\hline 56485024 & 29.7 & 5.75 & 3022 & $e^{+} e^{-} \mu^{+} \mu^{-}$ \\
\hline
\end{tabular}


: Table D.1. HRS four-Jepton candidate events with pair maves $\geq 1 \mathrm{GeV} / \mathrm{c}^{3}$ (coni.)

\begin{tabular}{|c|c|c|c|c|}
\hline Run\# Rec\# & $\begin{array}{c}\text { Energy } \\
\text { GeV }\end{array}$ & $\begin{array}{c}M_{\min } \\
\mathrm{GeV} / \mathrm{c}^{2}\end{array}$ & Track ID & Event ID \\
\hline 57161650 & 27.9 & 4.55 & 2272 & $e^{+} e^{-} e^{+} e^{-}$ \\
\hline 57387052 & 27.5 & 3.16 & 0302 & \\
\hline 584510576 & 29.6 & 2.23 & 3282 & $e^{+} e^{-} \mu^{+} \mu^{-}$ \\
\hline 59196519 & 20.6 & 1.47 & 2283 & $e^{+} e^{-} \mu^{+} \mu^{-}$ \\
\hline 59313466 & 29.7 & 1.50 & 3022 & $e^{+} e^{-} \mu^{+} \mu^{-}$ \\
\hline 60525680 & 28.9 & 8.20 & 2227 & $e^{+} e^{-} e^{+} e^{-}$ \\
\hline 60911994 & 29.6 & 1.19 & 3023 & $e^{+} e^{-} \mu^{+} \mu^{-}$ \\
\hline 61509691 & 29.7 & 1.53 & 2332 & $e^{+} e^{-} \mu^{+} \mu^{-}$ \\
\hline 61945960 & 24.1 & 2.49 & 2302 & $e^{+} e^{-} \mu^{+} \mu^{-}$ \\
\hline 62129814 & 22.3 & 2.60 & 0232 & $e^{+} e^{-} \mu^{+} \mu^{-}$ \\
\hline 62346807 & 25.4 & 2.80 & 2292 & $e^{+} e^{-} e^{+} e^{-}$ \\
\hline 62421640 & 32.1 & 1.07 & 2280 & \\
\hline 63065269 & 25.8 & 1.67 & 2728 & \\
\hline 63636746 & 29.5 & 1.94 & 0233 & $e^{+} e^{-} \mu^{+} \mu^{-}$ \\
\hline 64297832 & 28.6 & 3.77 & 2522 & $e^{+} e^{-} e^{+} e^{-}$ \\
\hline 645010383 & 29:: & 1.20 & 3322 & $e^{+} e^{-} \mu^{+} \mu^{-}$ \\
\hline 64632311 & 27.9 & 1.67 & 0222 & $e^{+} e^{-} e^{+} e^{-}$ \\
\hline 658211771 & 30.8 & 2.93 & 5200 & \\
\hline 66866531 & 29.4 & 1.08 & 3427 & \\
\hline
\end{tabular}


$\therefore$ Table D.1. BRS four-lepton candidale events fith pair manes $\geq 1 \mathrm{GeV} / \mathrm{c}^{2}$ (cont.)

\begin{tabular}{|c|c|c|c|c|}
\hline Run\# Rec\# & $\begin{array}{c}\text { Energy } \\
\text { GeV }\end{array}$ & $\begin{array}{c}M_{\min } \\
\mathrm{GeV} / \mathrm{c}^{2}\end{array}$ & Track ID & Event ID \\
\hline 69768982 & 20.0 & 2.26 & 3300 & \\
\hline 71255214 & 29.1 & 1.61 & 2279 & \\
\hline 7199743 & 29.3 & 1.11 & 2230 & $e^{+} e^{-} \mu^{+} \mu^{-}$ \\
\hline 72946904 & 25.5 & 6.43 & 2234 & \\
\hline 731110910 & 21.8 & 4.37 & 2224 & \\
\hline 73826790 & 27.9 & 3.00 & 2222 & $e^{+} e^{-} e^{+} e^{-}$ \\
\hline $7396 \quad 1752$ & 21.5 & 1.58 & 2800 & \\
\hline 74093263 & 21.2 & 1.43 & 4344 & \\
\hline 7437893 & 27.3 & 1.04 & 2094 & \\
\hline 746312190 & 29.1 & 2.35 & 3333 & $\mu^{+} \mu^{-} \mu^{+} \mu^{-}$ \\
\hline $7596 \quad 14149$ & 29.9 & 1.66 & 2202 & $e^{+} e^{-} e^{+} e^{-}$ \\
\hline 76411298 & 33.1 & 1.38 & 0200 & \\
\hline 81643338 & 27.7 & 1.23 & 0277 & \\
\hline 82134672 & 30.7 & 1.03 & 2924 & \\
\hline 83023723 & 26.1 & 2.09 & 3274 & \\
\hline 84568514 & 24.3 & 1.12 & 8302 & $e^{+} e^{-} \mu^{+} \mu^{-}$ \\
\hline 85246170 & 25.8 & 1.34 & 5225 & $e^{+} e^{-} e^{+} e^{-}$ \\
\hline 86121591 & 27.1 & 1.26 & 2823 & $e^{+} e^{-} \mu^{+} \mu^{-}$ \\
\hline 86252975 & 25.0 & 3.09 & 3239 & $e^{+} e^{-} \mu^{+} \mu^{-}$ \\
\hline
\end{tabular}




\section{REFERENCES}

1. T. Kinoshita, CERN-TH-5097/88, July 1988.

2. A.S. Elkovskii, Novosibjrsk Inst. Nucl. Phys. Acad. Sci--88-050, June 1988.

3. A. Davies, S. Brodsky, SLAC-PUB-4498, Dec. 1987; J. Grifols, A. Mendez, Phys. Rev. D26, 1809 (1982); A. Mendez, F. Orteu, Phys. Lett. 163B, 167 (1985) and references therein. A general review of recent studies beyond the standard model can be found in Proceedings of the 1987 International Symposium on Lepton and Photon Interactions at High Energies, DESY, Hamburg.

4. F. A. Berends, P. A. Daverveldt and R. Kleiss, Comp. Phys. Comm. 40 (1986) 285.

5. F. A. Berends, P. A. Daverveldt and R. Kleiss, Nucl. Phys. B253 (1985) 441.

6. F. A. Berends, P. A. Daverveldt and R. Kleiss, Comp. Phys. Comm. 40 (1986) 309.

7. R. Kleiss, Nucl. Pbys. B241 (1984) 61 .

8. MARK J Collaboration, DESY-85-073 (1985); JADE Collaboration, Z Phys. C30, 545 (1986); ASP Collaboration, SLAC-Report-337 (1989).

9. CELLO Collaboration, DESY-84-103 (1984).

10. CELLO Collaboration, Z Phys. C43, 1 (1989).

11. M. Alam, et al, SLAC-Proposal-PEP-005, December, 1976.

12. B. W. LeClire, Ph.D. thesis, Stanford University, SLAC-Report-321, 1987. 
13. R. H. Schindler, et al., Phys. Rev. D24 (1981) 78.

14. J. Rees, et al., Particle Accelerator Conference, Chicago, 1977, p. 1836.

15. J. A. Jaros, in Proceedings of the International conference on Instrumentation for Colliding Beam Physics, edited by W. Ash (Stanford Linear Accelerator Center, Stanford, CA, 1982), pp. 29-33.

16. W. Davies-White, et al., Nucl. Instr. and Meth. 160 (1979) 227.

17. G. Abrams, et al, IEEE Trans. Nucl. Sci. NS-25 (1978) 309; NS-27, 59 (1980).

18. M. Derrick, et al., SLAC-Proposal-PEP-12, October, 1977.

19. D. Bender, et al., Phys. Rev. D30 (1984) 151.

20. R. Ng, Ph.D. thesis, Purdue Uziversity, 1987.

21. P. Baringer, et al., Nucl. Jastr. and Meth. A254 (1987) 542.

22. D. Rubin, et al., Nucl. Instr. and Meth. 203 (1982) 119.

23. G. Baranko, et al., Nucl. Instr. and Meth. 169 (1980) 413.

24. J. Loos, et al., Nucl. Instr. and Meth. A249 (1986) 185.

25. Christiaan Huygens, Traité de la Lumière, Leiden, 1690.

26. H. Euler and B. Kockel, Naturwissenschaften 23 (1935) 246.

27. H. Euler, Ann. Phys. 26 (3936) 398.

28. A. Akbiezer, Phys. Zs. Soviet Union 11 (1937) 263.

29. L. Landau and E. Lifshite, Phys. Zs. Soviet Union 6 (1934) 244.

30. J. Smith, J. A. M. Vermaseren and G. Grammer, Phys. Rev. D15 (197i) 3280 .

31. courtesy of T. Barklow.

32. T. Sjostrand, Comp. Phys. Comm. 39 (1986) 347. 
33. courtesy of $P$. Burchat.

34: C. Peterson et al., Phys. Rev. D27 (1983) 105.

35. courtesy of A. Petersen, P. Sheldon and A. Weir.

36. F.A. Berends, et al. (CALCUL Collaboration), Nucl. Phys. B239 (1984) 395, calculation of the matrix elements of the process; Monte Carlo program by C.Hawkins (ASP collaboration), Ph.D. thesis, Stanford University, SLACReport-337, 1989.

37. courtesy of J. Repond.

38. M. E. Nelson, Ph.D. thesis, Lawrence Berkeley Laboratory Report No. LBL16724, 1983 .

39. F.A. Berends, P. Daverveldt, and R. Kleiss, Nucl. Phys. B253, 421 (1985); Comp. Pbys. Comm. 40, 271 (1986). They have only treated the radiative corrections of multiperipheral four-lepton production which peaks at small angles.

40. E. A. Kuraev and V. S. Fadin, Sov. J. Nucl. Phys. 41 (1985) 466.

41. P. R. Burchat, Ph.D. thesis, Stanford University, SLAC-Report-292, 1986.

42. courtesy of $R$. Tschirhart.

43. G. Cochard, S. Ong, Phys. Rev. D19 (1979) 810.

44. M. Defrise, Z. Phys. C9 (1981) 41.

45. W. L. Van Neerven and J. A. M. Vermaseren, Nucl. Phys. B238 (1984) 73.

46. P. Burchat's tapes SC2882 and SC2172 which contain $9278 e^{+} e^{-\gamma}$ and $\mu^{+} \mu^{-} \gamma$ event 5. 